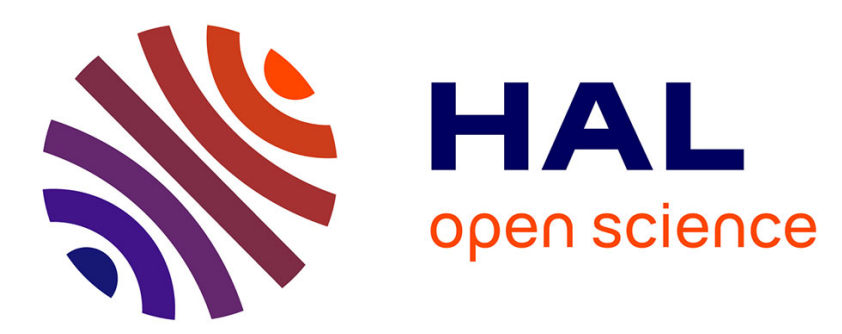

\title{
A complete list of symmetry adapted expressions to the fourth power for compact bending potentials in molecules with C3v and Td symmetry from a general symbolic algebra program \\ Roberto Marquardt, Kenneth Sagui
}

\section{To cite this version:}

Roberto Marquardt, Kenneth Sagui. A complete list of symmetry adapted expressions to the fourth power for compact bending potentials in molecules with C3v and Td symmetry from a general symbolic algebra program. Molecular Physics, 2007, 105 (09), pp.1157-1169. 10.1080/00268970701244783 . hal-00513085

\section{HAL Id: hal-00513085 \\ https://hal.science/hal-00513085}

Submitted on 1 Sep 2010

HAL is a multi-disciplinary open access archive for the deposit and dissemination of scientific research documents, whether they are published or not. The documents may come from teaching and research institutions in France or abroad, or from public or private research centers.
L'archive ouverte pluridisciplinaire HAL, est destinée au dépôt et à la diffusion de documents scientifiques de niveau recherche, publiés ou non, émanant des établissements d'enseignement et de recherche français ou étrangers, des laboratoires publics ou privés. 


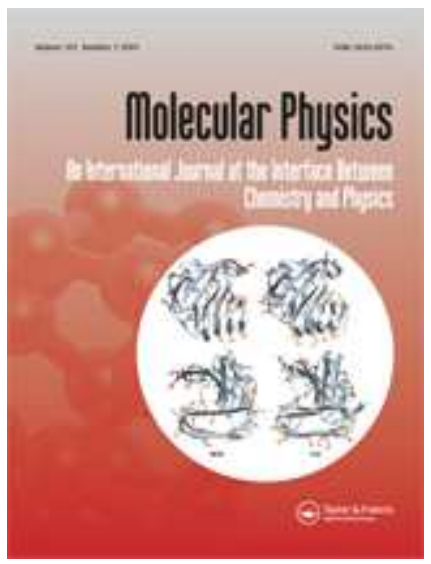

\section{A complete list of symmetry adapted expressions to the fourth power for compact bending potentials in molecules with C3v and Td symmetry from a general symbolic algebra program}

\begin{tabular}{|c|c|}
\hline Journal: & Molecular Physics \\
\hline Manuscript ID: & TMPH-2006-0112.R1 \\
\hline Manuscript Type: & Full Paper \\
\hline $\begin{array}{r}\text { Date Submitted by the } \\
\text { Author: }\end{array}$ & 24-Jan-2007 \\
\hline Complete List of Authors: & $\begin{array}{l}\text { Marquardt, Roberto; Université Louis Pasteur, Institut de } \\
\text { Chimie LC3-UMR7177-CNRS/ULP } \\
\text { Sagui, Kenneth; Université de Marne-la-Vallée }\end{array}$ \\
\hline Keywords: & $\begin{array}{l}\text { symmetry adapted coordinates, reduction of tensor products, } \\
\text { potential energy surfaces }\end{array}$ \\
\hline \multicolumn{2}{|c|}{$\begin{array}{l}\text { Note: The following files were submitted by the author for peer review, but cannot be converted } \\
\text { to PDF. You must view these files (e.g. movies) online. }\end{array}$} \\
\hline $\begin{array}{l}\text { TMPH-2006-0112.R1.tex } \\
\text { TMPH-2006-0112.R1-sup.tex }\end{array}$ & \\
\hline
\end{tabular}

\section{s ScholarONE" \\ Manuscript Central}




\section{Prepared for publication in Molecular Physics}

Pavel Rosmus special issue with 10 manuscript pages, 2 Tables, 2 Appendices

version January 24, 2007

\footnotetext{
* present address and email: roberto.marquardt@chimie.u-strasbg.fr correspondence and proofs to Prof. Roberto Marquardt Laboratoire de Chimie Quantique - Institut de Chimie - LC3 UMR 7177 CNRS/ULP Université Louis Pasteur - 4, rue Blaise Pascal - 67000 STRASBOURG - France Fax 0(033)3 90241589
} 


\begin{abstract}
An algorithm is proposed to perform the reduction of direct product representations of finite groups and, in particular, to perform the complete symmetry adaption of power representations, which may be used to obtain compact analytical representations of potential energy surfaces following an idea described in [R. Marquardt and M. Quack, J. Chem. Phys. 109, 10628 (1998)]. The algorithm is general in the sense that it can be applied to any finite group being characterized by its set of irreducible representations. It is automatic in the sense that, in case the reduction yields multiple degenerate irreducible subspaces of the same species, all degenerate irreducible subspaces are obtained with a coherent phase relation. The algorithm is based on the standard reduction rule of traditional representation theory. A symbolic algebra computer program based on MAPLE is presented and applied here to obtain the complete list of symmetry adapted expressions of bond angle coordinates to up to the fourth power in all irreducible representations of the $\mathbf{C}_{3 \mathrm{v}}$ and $\mathbf{T}_{\mathrm{d}}$ point groups.
\end{abstract}

PACS: $31.15 . \mathrm{Hz}, 31.50 .-\mathrm{x}, 02.70 . \mathrm{Wz}, 02.20 . \mathrm{Hj}$

Keywords: symmetry adapted coordinates, reduction of tensor products, potential energy surfaces 


\section{Introduction}

The derivation of potential energy surfaces from $a b$ initio calculations, and their accurate analytical representation in terms of functions of internal vibrational or reaction coordinates, has been of central interest to the work of Pavel Rosmus, the references [1-4] being some examples thereof. Analytical representations are quite often based on polynomial functions, which have the drawback of being interpolation formulae for ab initio derived energy points on the potential energy surface, rather than extrapolation formulae, and for adequate representations of large amplitude molecular rearrangements in highly anharmonic potentials, such as in $\mathrm{B}_{4}[5]$ and $\mathrm{SiC}_{3}$ [6], many high order polynomial terms are commonly needed.

While the description of anharmonicity of bond stretching potentials is well settled, e.g. with the use of Morse potentials [7] or alike, anharmonicity of bending potentials is considerably more difficult to describe analytically, in particular for highly symmetric molecules such as $\mathrm{B}_{4}$. We have developed a method for deriving potential energy forms for bending vibrations that allow for the accurate description of large amplitude molecular motion and applied it to derive global analytical representations of methane [8,9] and ammonia [10]. The derivation of these forms relies on extensive use of representation theory, for which a symbolic algebra program was developed. In particular, representation theory is used in this program to derive irreducible representations of products of coordinates that are used to build up compact potential energy forms $[8,10]$.

Both the derivation of the formulae and the program have not yet been published. The purpose of the present paper is to fill this gap and to show potential applications of the code to obtain symmetry adapted expressions of coordinates and products thereof for use in the derivation of potential energy surfaces for large amplitude molecular vibrations.

The program is based on a general reduction algorithm and is implemented as a symbolic algebra code within the MAPLE [11] program package. In the present version, the code is adapted to treat real representation spaces of some important finite point groups. Extension to other point groups has not yet been programmed, but is straightforward. The program is made available as supplementary material and the list of groups included 
so far is given in a user guide.

The paper is organized as follows: In section 2 the basic steps used in the program are described, first by giving a brief review of the well known reduction procedure in section 2.2; then by explaining, in section 2.3 , a new idea used to perform also a complete reduction in the representation space of the symmetric group, to reduce symmetric powers of representations, in addition to the reduction in the molecular point group. Section 3 describes, as an application, results for all symmetry adapted coordinates of power representations to up to the fourth power that arise in the context of the derivation of analytical potential energy surfaces for polyatomic molecules having $\mathbf{C}_{3 \mathrm{v}}$ and $\mathbf{T}_{\mathrm{d}}$ point group symmetries. The results obtained finally are formulated in such a way that they can be interpreted independently of the underlying physical problem of the representation of potential energy surfaces and may indeed be used quite generally.

\section{Theory}

\subsection{Representation theory}

Symmetry is treated mathematically in group theory, and the representation of groups in vector spaces, the subject of representation theory, plays a major role in the study of the physical laws of nature [12]. A key question in this study is the reducibility of representations into simple sets of irreducible representations that will serve to label and to classify typical properties of the physical systems obeying these laws.

The reduction of a representation is performed by a linear transformation of the representation vector space such as to yield subspaces that are invariant under operations of the symmetry group. This procedure is called symmetry adaption and deriving symmetry adapted coordinates is one important application of representation theory. While the existence of the linear transformation is proven in representation theory, and the necessary tools to reduce representations are in principle well established [12-14], it is often quite difficult to calculate the transformation matrix in practice. Such a work can be performed more easily using a symbolic algebra computer program. 
1

2

3

4

5

6

7

8

9

A commonly used procedure to obtain the transformation matrix for the reduction of a representation is to apply projection operators and ortho-normalize the projected vectors. Ortho-normalization yields vectors modulo a constant phase factor. In addition, projection into degenerate irreducible representation subspaces and subsequent orthonormalization yields a set of orthonormal vectors that is unique only to up to an unitary transformation in the corresponding subspace. Transformation matrices resulting from the projection and ortho-normalization steps can therefore be different matrices, rather than unique, which are similar to each other. The non-uniqueness in the ortho-normalization has no consequences for non-degenerate irreducible representations. However, if a representation is to be reduced into multiple degenerate irreducible representations of the same species, the labeling of the irreducible vectors is ambiguous, and the relative phases of corresponding irreducible vectors may lack coherence.

The present work uses a standard reduction algorithm, which is based on the "generalized projection operator" [14, Eq. (4.52)] (see also Eq (3-184) in [13]). This algorithm is summarized in section 2.2 .

We wish to note that the present code does not replace traditional methods for reducing powers of representations based on hand computation and using published tables of Clebsh-Gordan coefficients and for the reduction of direct products (see e.g. [15]). However, for those groups contained in the current version of the code, the computation is easier than by hand.

We also mention that the present algorithm does not solve the "missing label problem", since ambiguities remain because of the non-uniqueness in the ortho-normalization procedure. For a discussion of this problem and alternative methods for the reduction of representations we refer to [16]. However, this algorithm offers a straightforward means to obtain a coherent set of reduced representation spaces and symmetry adapted coordinates. 


\subsection{Reduction procedure}

Representation theory asserts first that $[12,13]$, for a given finite symmetry group $\mathcal{G}$ (we shall constrain our considerations to finite groups in this paper) there is a finite number $N_{\text {irr }}$ of irreducible representations $\mathcal{D}^{(\mu)}=\left\{\underline{\underline{D}}^{(\mu)}(g) \mid g \in \mathcal{G}\right\}$ of $\mathcal{G}$, each one defined in a $\mathbb{C}$-vector space $V_{d_{\mu}}$ of dimension $d_{\mu}\left(\mu=1, \ldots, N_{\text {irr }}\right) ; \underline{\underline{D}}^{(\mu)} \in V_{d_{\mu}} V_{d_{\mu}}$ are $d_{\mu} \times d_{\mu}$ unitary matrices. Secondly, if $\Gamma=\{\boldsymbol{\Gamma}(g) \mid g \in \mathcal{G}\}$ is any representation of $\mathcal{G}$ in a $\mathbb{C}$-vector space $V_{n}$ of dimension $n\left(\boldsymbol{\Gamma} \in V_{n}^{V_{n}}\right.$ are $n \times n$ unitary matrices $)$, then to reduce such a representation into direct sums of irreducible representations means to find a unitary $n \times n$ matrix $Z$, such that the transformed matrices

$$
\boldsymbol{\Gamma}^{\mathrm{red}}(g)=\boldsymbol{Z}^{\dagger} \cdot \boldsymbol{\Gamma}(g) \cdot \boldsymbol{Z}
$$

where the dagger indicates Hermitean conjugation, are block-diagonal matrices composed of blocks for each irreducible representation and, within a given irreducible representation block labelled by the symbol $\mu$, of $\gamma_{\mu}$ identical block-diagonal matrices $\underline{\underline{D}}^{(\mu)}(g)$, for all $g \in \mathcal{G}$. The number $\gamma_{\mu}$ is also called reduction factor of the irreducible representation $\mathcal{D}^{(\mu)}$ in $\Gamma$.

The transformation matrix $\boldsymbol{Z}$ may be composed of column vectors $\boldsymbol{z}^{(\mu ; k ; \alpha)}$, of column length $n$, where the superindices indicate the number $\mu$ of the irreducible representation, and running indices $k=1, \ldots, \gamma_{\mu}$, and $\alpha=1, \ldots, d_{\mu}$. The transformation matrix $\boldsymbol{Z}$ can then be obtained on the basis of "generalized projection operators" [13, equation (3-184) therein] $\boldsymbol{P}^{(\mu)}\left(\alpha, \alpha^{\prime}\right)$, defined here as

$$
\boldsymbol{P}^{(\mu)}\left(\alpha, \alpha^{\prime}\right)=\frac{d_{\mu}}{|\mathcal{G}|} \sum_{g \in \mathcal{G}} D_{\alpha \alpha^{\prime}}^{(\mu)^{*}}(g) \cdot \boldsymbol{\Gamma}(g),
$$

where $D_{\alpha \alpha^{\prime}}^{(\mu)}(g)$ is an element of the irreducible representation matrix $\underline{\underline{D}}^{(\mu)}(g)$. A reduction algorithm using these operators could then look as follows:

Step 1: For a given value of $\alpha$ (i.e. $\alpha=1$ ), get column vectors $\boldsymbol{z}^{(\mu ; k ; \alpha)}$ by first applying $\boldsymbol{P}^{(\mu)}(\alpha, \alpha)$ from Eq. (2) on all basis vectors of $V_{n}$, and then applying an ortho-normalization procedure, such as that of Gram-Schmidt [17], on the resulting projected vector space. In practice, one has to ortho-normalize all column vectors 
of $\boldsymbol{P}^{(\mu)}(\alpha, \alpha)$ to obtain $\gamma_{\mu}$ ortho-normalized vectors $\boldsymbol{z}^{(\mu ; k ; \alpha)}, k=1, \ldots, \gamma_{\mu}$. An alternative to the ortho-normalization procedure would be the diagonalization of an appropriate totally symmetric hermitian operator $[18,19]$.

Step 2: For $k$ from 1 to $\gamma_{\mu}$, and for $\beta \neq \alpha$ (i.e. for $\beta$ from 2 to $d_{\mu}$ ), get column vectors $\boldsymbol{z}^{(\mu ; k ; \beta)}$ by applying $\boldsymbol{P}^{(\mu)}(\beta, \alpha)$ from Eq. (2) on $\boldsymbol{z}^{(\mu ; k ; \alpha)}$,

$$
\boldsymbol{z}^{(\mu ; k ; \beta)}=\boldsymbol{P}^{(\mu)}(\beta, \alpha) \cdot \boldsymbol{z}^{(\mu ; k ; \alpha)},
$$

within individual manifolds of equal $k$-labels.

Based on the orthogonality relations of irreducible representation matrices [13, Eq (3143)], it can be shown that the $n$ vectors obtained in this way define indeed an orthonormal basis:

$$
\boldsymbol{z}^{(\mu ; k ; \alpha)^{\dagger}} \cdot \boldsymbol{z}^{\left(\mu^{\prime} ; k^{\prime} ; \alpha^{\prime}\right)}=\delta_{\mu \mu^{\prime}} \delta_{k k^{\prime}} \delta_{\alpha \alpha^{\prime}}
$$

For a given coordinate vector $\boldsymbol{x} \in V_{n}$, the expressions

$$
s_{\alpha}^{(\mu ; k)}=\boldsymbol{z}^{(\mu ; k ; \alpha)^{\dagger}} \cdot \boldsymbol{x} \quad\left(\alpha=1, \ldots, d_{\mu}\right)
$$

form a coherent set of $d_{\mu}$ symmetry adapted coordinates, for a given value of $\mu$ and $k$.

\subsection{Direct product representations}

In this section we use a simple superscript ${ }^{(n)}$ on representation symbols in order to assign the dimension of the representation. Boldfaced symbols are used for matrices; a simple superscript indicates the rank of squared matrices, a double superscript indicates the number of rows and columns of rectangular matrices. Let $\Gamma^{(n)}$ be a product representation in a vector space $V_{n}$, which we interpret as a tensor space, for instance $V_{n}=V_{n_{1}} \otimes V_{n_{2}}$, with $n=n_{1} \times n_{2}$, and $\Gamma^{(n)}=\Gamma^{\left(n_{1}\right)} \otimes \Gamma^{\left(n_{2}\right)}$. Elements of representation matrices $\boldsymbol{\Gamma}^{(n)}(g) \in \Gamma^{(n)}$ are related to elements of representation matrices $\boldsymbol{\Gamma}^{\left(n_{1}\right)}(g) \in \Gamma^{\left(n_{1}\right)}$ and $\boldsymbol{\Gamma}^{\left(n_{2}\right)}(g) \in \Gamma^{\left(n_{2}\right)}$ as follows:

$$
\Gamma_{k_{r} k_{c}}^{(n)}(g)=\Gamma_{i_{1 r}\left(k_{r}\right) i_{1 c}\left(k_{c}\right)}^{\left(n_{1}\right)}(g) \Gamma_{i_{2 r}\left(k_{r}\right) i_{2 c}\left(k_{c}\right)}^{\left(n_{2}\right)}(g)
$$

where $k_{r}, k_{c}=1, \ldots, n, i_{1 r}, i_{1 c}=1, \ldots, n_{1}$ and $i_{2 r}, i_{2 c}=1, \ldots, n_{2}$; the subindexes $r$ and $c$ differ row and column indexes. The algorithm proposed in section 2.2 may be used to reduce such representations. 
In the special case when $\Gamma^{\left(n_{1}\right)}=\Gamma^{\left(n_{2}\right)}$, there is an additional symmetry related to the permutation of identical factors. It is then possible to consider reducing the representation in the symmetric group $\mathbf{S}_{2}$, in addition to reducing it in $\mathcal{G}$. Indeed, for commutative algebras only the $\mathrm{A}_{1}$ symmetry adapted coordinates in $\mathbf{S}_{2}$ will have non-vanishing components in $V_{n}\left(\mathrm{~A}_{1}\right.$ is the totally symmetric or trivial representation). Therefore, before reducing $\Gamma^{(n)}$ in $\mathcal{G}$, it is useful to reduce $V_{n}$ to the space spanned by $\mathrm{A}_{1}$-vectors.

In general, a power direct product representation of order $m(m=2,3,4, \ldots), \Gamma^{\left(n^{m}\right)}=$ $\Gamma^{(n)^{\otimes m}}$, of any group $\mathcal{G}$ gives also rise to a representation $\Sigma^{\left(n^{m}\right)}$ of the symmetric group $\mathbf{S}_{\mathrm{m}}$, the construction of which is described below. A product representation matrix element of order $m$ is calculated as

$$
\Gamma_{k_{r} k_{c}}^{\left(n^{m}\right)}(g)=\Gamma_{i_{1 r}\left(k_{r}\right) i_{1 c}\left(k_{c}\right)}^{(n)}(g) \cdot \ldots \cdot \Gamma_{i_{m r}\left(k_{r}\right) i_{m c}\left(k_{c}\right)}^{(n)}(g) .
$$

Let $I_{m}=\left(i_{1}, \ldots, i_{m}\right)$ be a $m$-tuple of indexes $i_{j}=1, \ldots, n(j=1, \ldots, m)$ that describes a basis state vector of the tensor space $V_{n^{m}}$. There are $n^{m} m$-tuples of this form:

$$
I_{m}(k)=\left(i_{1}(k), \ldots, i_{m}(k)\right) \quad k=1, \ldots, n^{m}
$$

$i_{j}(k)=1, \ldots, n ; j=1, \ldots, m$. We call the assignment $k \rightarrow I_{m}(k)$ in Eq. (8) a product representation key. The definition of the key implies the calculation of the elements of the product representation matrix from the factor matrix elements in Eq. (7).

Consider a specific key component $I_{m}(k)$. A given operation $p \in \mathbf{S}_{\mathrm{m}}$ performs a permutation of indexes $i_{j}$ in $I_{m}(k)$ which will correspond in a unique way to a new key component $I_{m}\left(k^{\prime}\right)$. Representation matrix elements $\Sigma_{k k^{\prime}}^{\left(n^{m}\right)}(p)$ will therefore have the values 1 or 0 , depending on whether $k$ and $k^{\prime}$ are connected by $p$ or not. We calculate such matrices analytically using the regular representation of $\mathbf{S}_{\mathrm{m}}$ (the "Turmdarstellung").

$\Sigma^{\left(n^{m}\right)}$ is reducible in $\mathbf{S}_{\mathrm{m}}$, and the reduction will always contain the $\mathrm{A}_{1}$-representation. The algorithm proposed in section 2.2 may be used to to reduce this representation as well. A simpler way of obtaining the $A_{1}$-subspace of any representation space is, however, to use the projection operator introduced by Wigner [12, Eq. (12.12a) therein]. In practice, we proceed as follows:

Step 1: Let $\gamma$ be the multiplicity of the $A_{1}\left(S_{m}\right)$-representation of the Symmetric group 
$\mathbf{S}_{\mathrm{m}}$ in the representation $\Sigma^{\left(n^{m}\right)}$, and let $V_{\gamma}$ be the $\mathrm{A}_{1}\left(\mathbf{S}_{\mathrm{m}}\right)$ invariant subspace of $V_{n^{m}}$. This subspace is defined in $V_{n^{m}}$ by the matrix $\boldsymbol{T}^{\left(n^{m} ; \gamma\right)}$, which has $\gamma$ orthonormal columns and $n^{m}$ rows. Calculate $\boldsymbol{T}^{\left(n^{m} ; \gamma\right)}$ from the orthonormalization of

$$
\boldsymbol{P}_{\mathrm{A}_{1}\left(\mathbf{S}_{\mathrm{m}}\right)}^{\left(n^{m}\right)}=\sum_{g \in \mathbf{S}_{\mathrm{m}}} \boldsymbol{\Sigma}^{\left(n^{m}\right)}(g)
$$

Then reduce $\Gamma^{(n)^{\otimes m}}$ to obtain the representation $\Gamma^{(\gamma)}$ of $\mathcal{G}$ in this subspace, e.g. for all $g \in \mathcal{G}$, calculate representation matrices

$$
\boldsymbol{\Gamma}^{(\gamma)}(g)=\boldsymbol{T}^{\dagger}\left(\gamma ; n^{m}\right) \cdot \boldsymbol{\Gamma}^{\left(n^{m}\right)}(g) \cdot \boldsymbol{T}^{\left(n^{m} ; \gamma\right)}
$$

Step 2: Let then $Z^{(\gamma)}$ be the unitary matrix of rank $\gamma$ that reduces $\Gamma^{(\gamma)}$ in $\mathcal{G}$. This matrix is obtained as described in the procedure of section 2.2. $\mathcal{G}$-symmetry adapted coordinates $\boldsymbol{s}^{(\gamma)} \in V_{\gamma}$ are then calculated from Eq. (11), for a given coordinate vector $\boldsymbol{x}^{\left(n^{m}\right)} \in V_{n^{m}}:$

$$
\boldsymbol{s}^{(\gamma)}=\boldsymbol{Z}^{\dagger(\gamma)} \cdot \boldsymbol{T}^{\dagger\left(\gamma ; n^{m}\right)} \cdot \boldsymbol{x}^{\left(n^{m}\right)}
$$

As in section 2.2, components of $\boldsymbol{s}^{(\gamma)}$ may be partitioned into distinguished sets of coordinates that are internally coherent. These sets may be labelled according to the irreducible representation species $\mu$ of $\mathcal{G}$ and some additional multiplicity index $k$.

\section{Results}

The algorithm described in section 2.2 was used, as an application, to find all symmetry adapted expressions, according to Eq. (11), of products of symmetry adapted bending coordinates of methane [8,20] and ammonia [10]. The symmetry adapted coordinates used here are well known from spectroscopic studies [21]. They may as well be obtained using the general reduction algorithm of section 2.2 upon appropriate definition of representation matrices of the $\mathbf{T}_{\mathrm{d}}$ and $\mathbf{C}_{3 \mathrm{v}}$ groups in the space of bond angle coordinates.

The validity of these expressions was tested by checking that the numerically obtained matrices $\boldsymbol{\Gamma}^{\mathrm{red}}(g)$ (Eq. (1)) are exactly blockdiagonal, for all $g \in \mathcal{G}$, and have identical block elements given by the matrices $\underline{\underline{D}}^{(\mu)}$. This test allows to find inconsistencies in the 
computer algebra approach. Furthermore it is fundamental to ensure the coherence of all vectors. Coherent vectors are relevant for the reduction of tensor operators in many physical problems. For a further discussion on the relevance of coherent vectors for the derivation of potential energy surfaces see ref. [10].

All expressions given here are direct results from the numerical program, but were further simplified by hand computation, where constant multiplicative factors have been omitted.

\section{$3.1 \quad \mathrm{NH}_{3}$}

Ammonia $\left(\mathrm{NH}_{3}\right)$ has a $\mathrm{C}_{3 \mathrm{v}}$ point group structure at equilibrium (the stable stationary point on the potential energy surface). Symmetry adapted bending coordinates are [21]

$$
\begin{aligned}
S A 1 & =\frac{1}{\sqrt{3}}\left(\Delta \alpha_{12}+\Delta \alpha_{13}+\Delta \alpha_{23}\right) \\
S E & =\left\{\begin{array}{l}
S E a=\frac{1}{\sqrt{6}}\left(2 \Delta \alpha_{12}-\Delta \alpha_{13}-\Delta \alpha_{23}\right) \\
S E b=\frac{1}{\sqrt{2}}\left(\Delta \alpha_{13}-\Delta \alpha_{23}\right)
\end{array}\right.
\end{aligned}
$$

where $\Delta \alpha_{i j}=\alpha_{i j}-\alpha_{i j}^{\text {eq }}$ are displacements of bond angles $\alpha_{i j}$ between NH bonds $i$ and $j$ $(i, j=1, \ldots, 3)$ from equilibrium values $\alpha_{i j}^{\mathrm{eq}}$.

The second order product representation space has dimension 9. Reduction in $\mathbf{S}_{2}$ yields a six-dimensional space which splits into a two-dimensional space of type $\mathrm{A}_{1}$ and a twodimensional space of type E. Symmetry adapted coordinates are

$$
\begin{aligned}
S A 1^{\{2\}} & =S A 1^{2} \\
S A 1^{\{2\}} & =S E a^{2}+S E b^{2} \\
S E a^{\{2\}} & =(S E a-S E b)(S E b+S E a) \\
S E b^{\{2\}} & =-2 S E a S E b \\
S E a^{\{2\}} & =S A 1 S E a \\
S E b^{\{2\}} & =S A 1 S E b
\end{aligned}
$$

Product representation spaces of order 3 and 4 have dimensions 27 and 81, respectively. Table 1 summarizes the resulting reductions, first in $\mathbf{S}_{2}, \mathbf{S}_{3}$ and $\mathbf{S}_{4}$, then in $\mathbf{C}_{3 \mathrm{v}}$. Symmetry 
adapted coordinates are summarized in appendix A.

\section{$3.2 \mathrm{CH}_{4}$}

Methane $\left(\mathrm{CH}_{4}\right)$ has a $\mathbf{T}_{\mathrm{d}}$ point group structure at equilibrium. Symmetry adapted bending coordinates are [21]

$$
\begin{aligned}
& S A 1=\frac{1}{\sqrt{6}}\left(\Delta \alpha_{12}+\Delta \alpha_{13}+\Delta \alpha_{14}+\Delta \alpha_{23}+\Delta \alpha_{24}+\Delta \alpha_{34}\right) \\
& S E=\left\{\begin{aligned}
S E a & =\frac{1}{\sqrt{12}}\left(2\left(\Delta \alpha_{12}+\Delta \alpha_{34}\right)-\left(\Delta \alpha_{13}+\Delta \alpha_{24}+\Delta \alpha_{23}+\Delta \alpha_{14}\right)\right) \\
S E b & =\frac{1}{2}\left(\Delta \alpha_{13}+\Delta \alpha_{24}-\Delta \alpha_{23}-\Delta \alpha_{14}\right)
\end{aligned}\right. \\
& S F 2=\left\{\begin{array}{l}
S F x=\frac{1}{\sqrt{2}}\left(\Delta \alpha_{12}-\Delta \alpha_{34}\right) \\
S F y=\frac{1}{\sqrt{2}}\left(\Delta \alpha_{13}-\Delta \alpha_{24}\right) \\
S F z=\frac{1}{\sqrt{2}}\left(\Delta \alpha_{14}-\Delta \alpha_{23}\right)
\end{array}\right.
\end{aligned}
$$

where $\Delta \alpha_{i j}=\alpha_{i j}-\alpha_{i j}^{\mathrm{eq}}$ are displacements of bond angles $\alpha_{i j}$ between $\mathrm{CH}$ bonds $i$ and $j$ $(i, j=1, \ldots, 4)$ from equilibrium values $\alpha_{i j}^{\mathrm{eq}}$.

Product representation spaces of order 2, 3 and 4 have dimensions 36, 216 and 1296, respectively. Table 2 summarizes the resulting reductions, first in $\mathbf{S}_{2}, \mathbf{S}_{3}$ and $\mathbf{S}_{4}$, then in $\mathbf{T}_{\mathrm{d}}$. Symmetry adapted coordinates are summarized in appendix B. 


\section{Conclusions}

The study of physical laws of nature often involves representations of symmetry groups in vector spaces. The reduction of such representations into simple sets of irreducible representations exploits a maximum of information embedded in the symmetry properties of these laws, which allows to label and classify typical properties of the corresponding physical systems. For instance, the structure and dynamics of polyatomic molecules may be studied on the basis of a classification of the complicated underlying spectroscopic data using properties of the symmetry inherent to these systems, such as the permutation of identical atoms [22-24]. The complete permutation-inversion group is also central to the derivation of selection rules for reactive collisions [25]. More recently, a new method for obtaining analytical representations of potential energy surfaces for polyatomic molecules was introduced, which requires the calculation of coherent sets of symmetry adapted coordinates corresponding to degenerate irreducible representations in high order tensor spaces $[8,10]$.

While the mathematical tools to reduce representations are well established [12,13], it is often quite difficult and laborious to calculate the transformation matrices that yield the symmetry adapted coordinates in practice.

In the present work we have used a reduction algorithm based on projector operators such as the "generalized projection operator" [13]. The algorithm was implemented into a symbolic algebra program using the MAPLE [26] code, and applied to the problem of reducing direct product and power representations. The algorithm is general, as it can be used for any finite group defined by its set of irreducible representations. It is automatic in the sense that, in case the reduction yields multiple degenerate irreducible subspaces of the same species, all degenerate irreducible subspaces are obtained with a coherent phase relation. We note that the resulting standardization is similar to the one introduced for the construction of symmetry adapted vectors used in crystal- and ligand-field theory [27,28]. For the reduction of a power representation $\Gamma^{\otimes m}$ in commutative algebras, the algorithm includes also reduction in the symmetric group $\mathbf{S}_{\mathrm{m}}$. The code allows currently to treat some of the finite groups, including the $\mathbf{C}_{2 \mathrm{v}}, \mathbf{C}_{3 \mathrm{v}}$ and $\mathbf{T}_{\mathrm{d}}$ groups, and powers of representations thereof to up to $m=4$. An extension to include other groups is straightforward, 
and we plan to apply it to derive a global potential energy surface for $\mathrm{B}_{4}$, for which the $\mathbf{D}_{4 \mathrm{~h}}$ group will play an important role [5]. The code may be of quite general use and is made available as supplementary material [29].

\section{Acknowledgment}

We thank Dr. D. Luckhaus and Dr. G. Dhont for early discussions, as well as Dr. V. Boudon, Dr. C. Leroy and Prof. F. Michelot for help and discussions in the final stage of this project. This work is supported financially by grants from the French Ministry of Research. 


\section{References}

[1] U. Mänz, E. Reinsch, P. Rosmus, H. Werner, and S. O. Neil, J. Chem. Soc. Faraday Trans., 87, 1809-1814 (1991).

[2] W. Gabriel, G. Chambaud, P. Rosmus, S. Carter, and N. C. Handy, Mol. Phys., 81, 1445-1461 (1994).

[3] M. Rosenstock, P. Rosmus, E.-A. Reinsch, O. Treutler, S. Carter, and N. C. Handy, Mol. Phys., 93, 853-865 (1998).

[4] P. Rosmus, P. Palmieri, and R. Schinke, J. Chem. Phys., 117, 4871-4877 (2003).

[5] R. Linguerri, I. Navizet, P. Rosmus, S. Carter, and J. P. Maier, J. Chem. Phys., 122, 34301 (2005).

[6] R. Linguerri, P. Rosmus, and S. Carter, J. Chem. Phys., 125, 34305 (2006).

[7] P. M. Morse, Phys. Rev., 34, 57-64 (1929).

[8] R. Marquardt and M. Quack, J. Chem. Phys., 109, 10628-10643 (1998).

[9] R. Marquardt and M. Quack, J. Phys. Chem. A, 108, 3166-3181 (2004).

[10] R. Marquardt, K. Sagui, W. Klopper, and M. Quack, J. Phys. Chem. B, 109, 8439$8451(2005)$.

[11] Maple V Release 3 (ETH). Copyright (c) 1981-1994 by Waterloo Maple Software and the University of Waterloo.

[12] E. Wigner. Group Theory. Academic Press, New York, (1959).

[13] M. Hamermesh. Group Theory and Its Application to Physical Problems. AddisonWesley, Reading, Massachusetts, (1962).

[14] J. P. Elliot and P. G. Dawber. Symmetry in Physics. Oxford University Press, New York, (1979).

[15] S. L. Altmann and P. Herzig. Point-group theory tables. Clarendon, Oxford, (1994). 
[16] J.-Q. Chen, M.-J. Gao, and G.-Q. Ma, Rev. Mod. Phys., 57, 211-278 (1985).

[17] H.-J. Kowalsky. Lineare Algebra. de Gruyter, Berlin, New York, (1979).

[18] M. Rey, V. Boudon, C. Wenger, G. Pierre, and B. Sartakov, J. Mol. Spectrosc., 219, $313-325$ (2003).

[19] J.-P. Champion, G. Pierre, F. Michelot, and J. Moret-Bailly, Can. J. Phys., 55, $512-520(1977)$.

[20] R. Marquardt, , F. Mariotti, and M. Quack. Work in preparation.

[21] J. L. Duncan and I. M. Mills, Spectrochim. Acta, 20, 523-546 (1964).

[22] G. Herzberg. Molecular Spectra and Molecular Structure II. Infrared and Raman Spectra of Polyatomic Molecules. Van Nostrand Reinhold Co., New York, reprint(1991) edition, (1945).

[23] G. Herzberg. Molecular Spectra and Molecular Structure III. Electronic Spectra and Electronic Structure of Polyatomic Molecules. Van Nostrand Reinhold Co., New York, reprint(1991) edition, (1966).

[24] P. R. Bunker. Molecular Symmetry and Spectroscopy. Academic Press, New York, (1979).

[25] M. Quack, Mol. Phys., 34, 477-504 (1977).

[26] Maple V Release 10. Copyright (c) 2004-2005 by Maplesoft, a division of Waterloo Maple Software Inc.

[27] M. Kibler, Int. J. Quantum Chem., 3, 795-822 (1969).

[28] M. R. Kibler, J. Mol. Spectrosc., 62, 247-262 (1976).

[29] R. Marquardt and K. Sagui. REDTEN: A symbolic algebra program for the automatic reduction of powers of representations of finite groups. A hard copy version of the codes and user guide has been deposited with the British Library Document Supply Centre as Supplementary Publication No SUP ... (77 pages). 


\section{A $\quad \mathrm{C}_{3 \mathrm{v}}$-symmetry adapted expressions}

\section{A.1 Expressions of order 3}

The product representation

$$
\Gamma^{(3)}=\left(\mathcal{D}^{\left(\mathrm{A}_{1}\right)} \oplus \mathcal{D}^{(\mathrm{E})}\right)^{\otimes 3}
$$

reduces as

$$
\Gamma^{\mathrm{red}(3)}=3 \mathcal{D}^{\left(\mathrm{A}_{1}\right)} \oplus \mathcal{D}^{\left(\mathrm{A}_{2}\right)} \oplus 3 \mathcal{D}^{(\mathrm{E})} .
$$

Symmetry adapted expressions are:

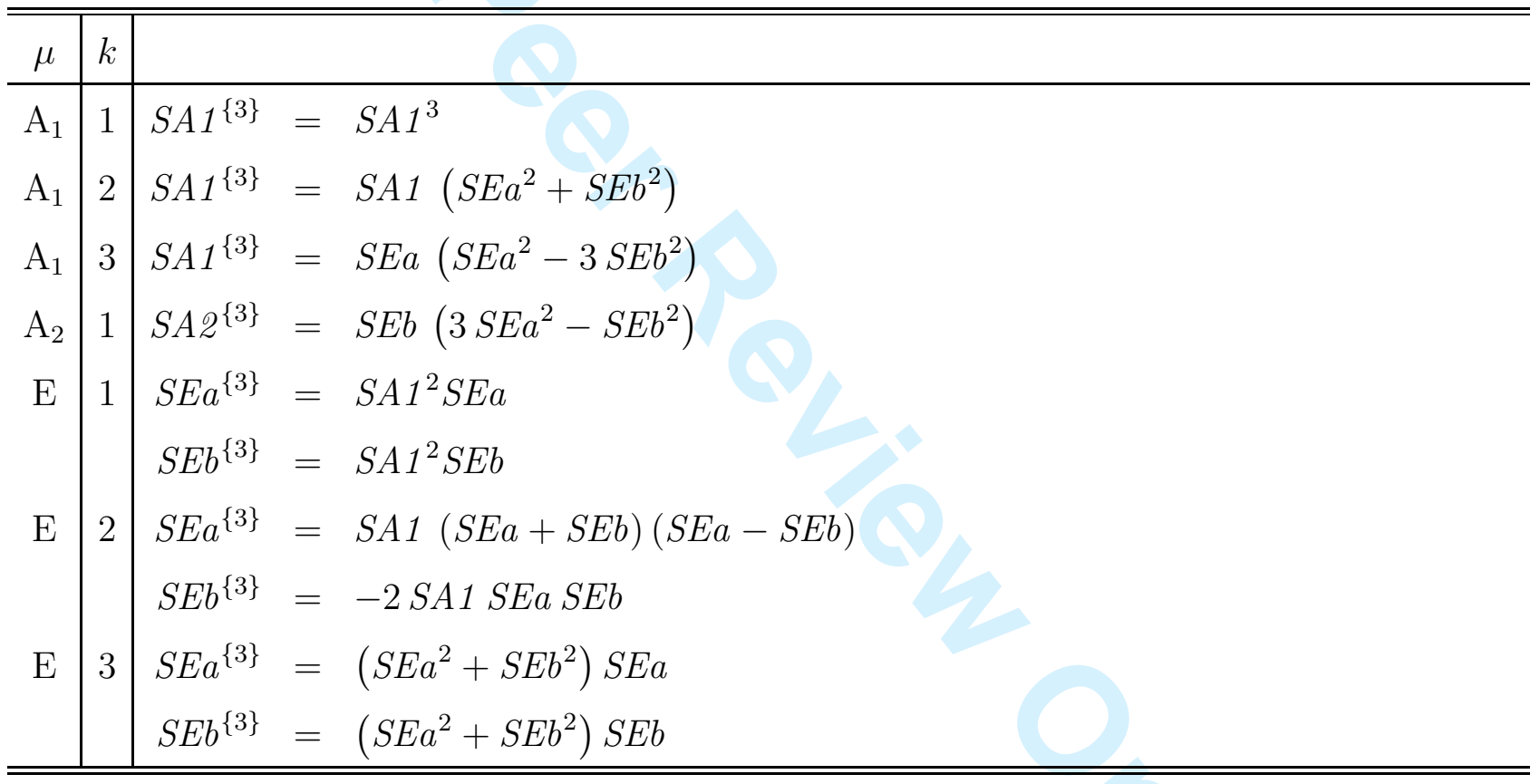




\section{A.2 Expressions of order 4}

The product representation

$$
\Gamma^{(4)}=\left(\mathcal{D}^{\left(\mathrm{A}_{1}\right)} \oplus \mathcal{D}^{(\mathrm{E})}\right)^{\otimes 4}
$$

reduces as

$$
\Gamma^{\mathrm{red}(4)}=4 \mathcal{D}^{\left(\mathrm{A}_{1}\right)} \oplus \mathcal{D}^{\left(\mathrm{A}_{2}\right)} \oplus 5 \mathcal{D}^{(\mathrm{E})}
$$

\begin{tabular}{|c|c|c|c|c|}
\hline$\mu$ & $k$ & & & \\
\hline $\mathrm{A}_{1}$ & 1 & $S A 1^{\{4\}}$ & $=$ & $S A 1^{4}$ \\
\hline $\mathrm{A}_{1}$ & 2 & $S A 1^{\{4\}}$ & $=$ & $\left(S E a^{2}+S E b^{2}\right) S A 1^{2}$ \\
\hline $\mathrm{A}_{1}$ & 3 & $S A 1^{\{4\}}$ & $=$ & $S A 1 S E a\left(S E a^{2}-3 S E b^{2}\right)$ \\
\hline $\mathrm{A}_{1}$ & 4 & $S A 1^{\{4\}}$ & $=$ & $\left(S E a^{2}+S E b^{2}\right)^{2}$ \\
\hline $\mathrm{A}_{2}$ & 1 & $S A 2^{\{4\}}$ & $=$ & $S A 1 S E b\left(3 S E a^{2}-S E b^{2}\right)$ \\
\hline $\mathrm{E}$ & 1 & $\begin{array}{l}S E a^{\{4\}} \\
S E b^{\{4\}}\end{array}$ & $\begin{array}{l}= \\
=\end{array}$ & $\begin{array}{l}1 / 2 \sqrt{3}(S E a-S E b)(S E a+S E b) S A 1^{2} \\
-\sqrt{3} S A 1^{2} S E a S E b\end{array}$ \\
\hline $\mathrm{E}$ & 2 & $\begin{array}{l}S E a^{\{4\}} \\
S E b^{\{4\}}\end{array}$ & $\begin{array}{l}= \\
=\end{array}$ & $\begin{array}{l}S A 1\left(S E a^{2}+S E b^{2}\right) S E a \\
S A 1\left(S E a^{2}+S E b^{2}\right) S E b\end{array}$ \\
\hline $\mathrm{E}$ & 3 & $\begin{array}{l}S E a^{\{4\}} \\
S E b^{\{4\}}\end{array}$ & $\begin{array}{l}= \\
=\end{array}$ & $\begin{array}{l}\frac{1}{300} \sqrt{10}\left(5 S E a^{2}-3 S E b^{2}+2 S E b^{2} \sqrt{6}\right)\left(5 S E a^{2}-3 S E b^{2}-2 S E b^{2} \sqrt{6}\right) \sqrt{3} \\
-1 / 15 \sqrt{10}\left(S E a^{2}+3 S E b^{2}\right) S E a S E b \sqrt{3}\end{array}$ \\
\hline $\mathrm{E}$ & 4 & $\begin{array}{l}S E a^{\{4\}} \\
S E b^{\{4\}}\end{array}$ & $\begin{array}{l}= \\
=\end{array}$ & $\begin{array}{l}\left(3 S E a^{2}-S E b^{2}\right) S E b^{2} \\
-\left(3 S E a^{2}-S E b^{2}\right) S E a S E b\end{array}$ \\
\hline $\mathrm{E}$ & 5 & $\begin{array}{l}S E a^{\{4\}} \\
S E b^{\{4\}}\end{array}$ & $\begin{array}{l}= \\
=\end{array}$ & $\begin{array}{l}S A 1^{3} S E a \sqrt{10} \\
S A 1^{3} S E b \sqrt{10}\end{array}$ \\
\hline
\end{tabular}

Symmetry adapted expressions are: 


\section{B $\quad T_{d}$-symmetry adapted expressions}

\section{B.1 Expressions of order 2}

The product representation

$$
\Gamma^{(2)}=\left(\mathcal{D}^{\left(\mathrm{A}_{1}\right)} \oplus \mathcal{D}^{(\mathrm{E})} \oplus \mathcal{D}^{\left(\mathrm{F}_{2}\right)}\right)^{\otimes 2}
$$

reduces as

$$
\Gamma^{\mathrm{red}(2)}=3 \mathcal{D}^{\left(\mathrm{A}_{1}\right)} \oplus 3 \mathcal{D}^{(\mathrm{E})} \oplus 1 \mathcal{D}^{\left(\mathrm{F}_{1}\right)} \oplus 3 \mathcal{D}^{\left(\mathrm{F}_{2}\right)}
$$

Symmetry adapted expressions are:

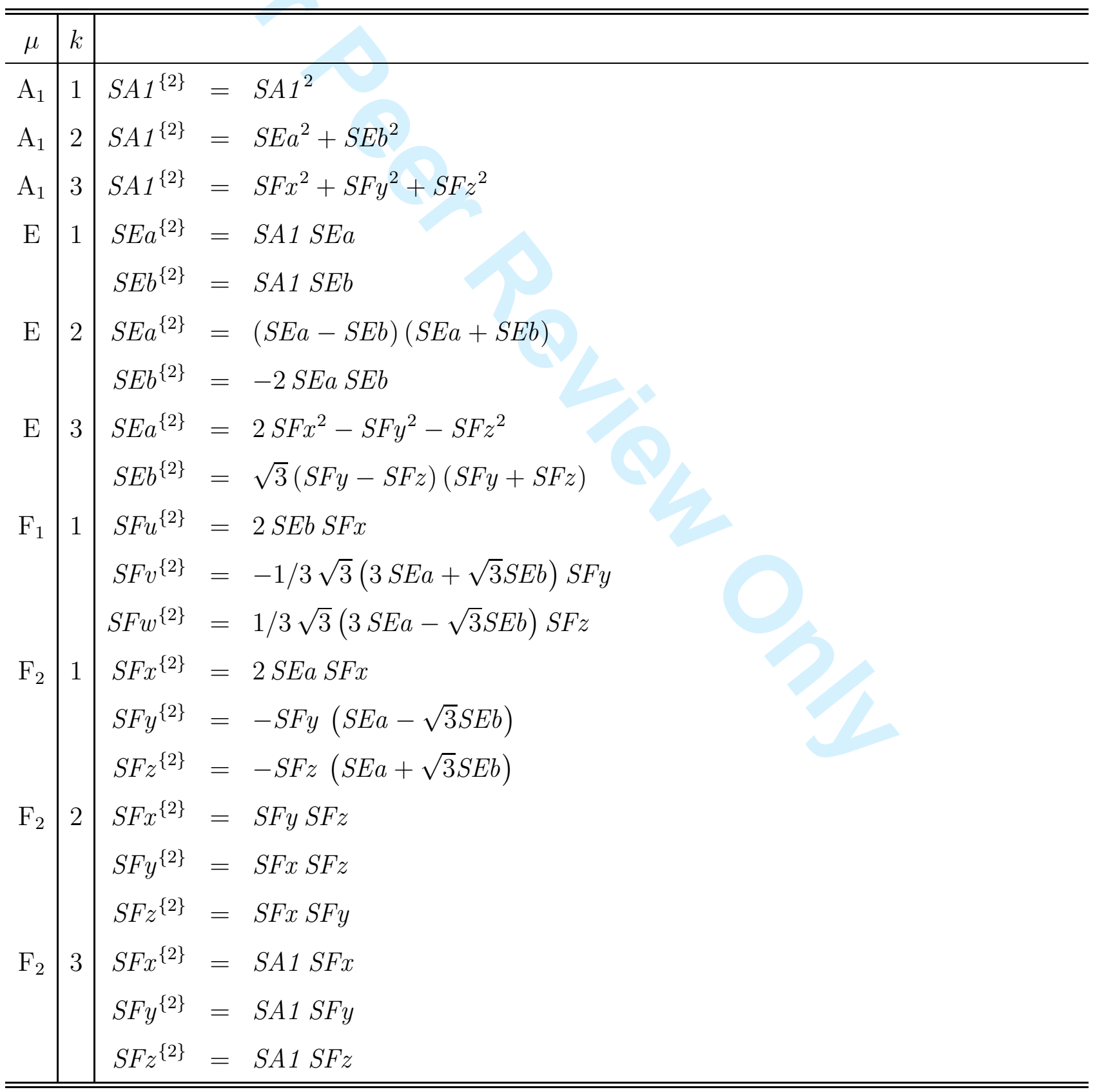




\section{B.2 Expressions of order 3}

The product representation

$$
\Gamma^{(3)}=\left(\mathcal{D}^{\left(\mathrm{A}_{1}\right)} \oplus \mathcal{D}^{(\mathrm{E})} \oplus \mathcal{D}^{\left(\mathrm{F}_{2}\right)}\right)^{\otimes 3}
$$

reduces as

$$
\Gamma^{\mathrm{red}(3)}=6 \mathcal{D}^{\left(\mathrm{A}_{1}\right)} \oplus 2 \mathcal{D}^{\left(\mathrm{A}_{2}\right)} \oplus 6 \mathcal{D}^{(\mathrm{E})} \oplus 4 \mathcal{D}^{\left(\mathrm{F}_{1}\right)} \oplus 8 \mathcal{D}^{\left(\mathrm{F}_{2}\right)}
$$

\begin{tabular}{|c|c|c|c|c|}
\hline$\mu$ & $k$ & & & \\
\hline $\mathrm{A}_{1}$ & 1 & $S A 1^{\{3\}}$ & $=$ & $S A 1^{3}$ \\
\hline $\mathrm{A}_{1}$ & 2 & $S A 1^{\{3\}}$ & $=$ & $S A 1\left(S E a^{2}+S E b^{2}\right)$ \\
\hline $\mathrm{A}_{1}$ & 3 & $S A 1^{\{3\}}$ & $=$ & $S A 1\left(S F x^{2}+S F y^{2}+S F z^{2}\right)$ \\
\hline $\mathrm{A}_{1}$ & 4 & $S A 1^{\{3\}}$ & $=$ & $S E a\left(-3 S E b^{2}+S E a^{2}\right)$ \\
\hline $\mathrm{A}_{1}$ & 5 & $S A 1^{\{3\}}$ & $=$ & $\begin{array}{l}S E a S F x^{2}-1 / 2 S E a S F y^{2}-1 / 2 S E a S F z^{2} \\
+1 / 2 \sqrt{3} S E b S F y^{2}-1 / 2 \sqrt{3} S E b S F z^{2}\end{array}$ \\
\hline $\mathrm{A}_{1}$ & 6 & $S A 1^{\{3\}}$ & $=$ & $S F x S F y S F z$ \\
\hline $\mathrm{A}_{2}$ & 1 & $S A 2^{\{3\}}$ & $=$ & $S E b\left(3 S E a^{2}-S E b^{2}\right)$ \\
\hline $\mathrm{A}_{2}$ & 2 & $S A 2^{\{3\}}$ & $=$ & $-2 \sqrt{3} S E b S F x^{2}+\sqrt{3} S E b S F y^{2}+\sqrt{3} S E b S F z^{2}+3 S E a S F y^{2}-3 S E a S F z^{2}$ \\
\hline $\mathrm{E}$ & 1 & $\begin{array}{l}S E a^{\{3\}} \\
S E b^{\{3\}}\end{array}$ & $\begin{array}{l}= \\
=\end{array}$ & $\begin{array}{l}S A 1(S E a-S E b)(S E a+S E b) \\
-2 S A 1 S E a S E b\end{array}$ \\
\hline $\mathrm{E}$ & 2 & $\begin{array}{l}S E a^{\{3\}} \\
S E b^{\{3\}}\end{array}$ & $\begin{array}{l}= \\
=\end{array}$ & $\begin{array}{l}-\sqrt{3} S E b S F z^{2}+\sqrt{3} S E b S F y^{2}+3 S E a S F y^{2}+3 S E a S F z^{2} \\
S E b S F z^{2}+S E b S F y^{2}-\sqrt{3} S E a S F z^{2}+4 S E b S F x^{2}+\sqrt{3} S E a S F y^{2}\end{array}$ \\
\hline $\mathrm{E}$ & 3 & $\begin{array}{l}S E a^{\{3\}} \\
S E b^{\{3\}}\end{array}$ & $\begin{array}{l}= \\
=\end{array}$ & $\begin{array}{l}S A 1^{2} S E a \\
S A 1^{2} S E b\end{array}$ \\
\hline $\mathrm{E}$ & 4 & $\begin{array}{l}S E a^{\{3\}} \\
S E b^{\{3\}}\end{array}$ & $\begin{array}{l}= \\
=\end{array}$ & $\begin{array}{l}S A 1\left(2 S F x^{2}-S F y^{2}-S F z^{2}\right) \\
\sqrt{3} S A 1(S F y+S F z)(S F y-S F z)\end{array}$ \\
\hline $\mathrm{E}$ & 5 & $\begin{array}{l}S E a^{\{3\}} \\
S E b^{\{3\}}\end{array}$ & $\begin{array}{l}= \\
=\end{array}$ & $\begin{array}{l}\left(S E a^{2}+S E b^{2}\right) S E a \\
\left(S E a^{2}+S E b^{2}\right) S E b\end{array}$ \\
\hline $\mathrm{E}$ & 6 & $\begin{array}{l}S E a^{\{3\}} \\
S E b^{\{3\}}\end{array}$ & $\begin{array}{l}= \\
=\end{array}$ & $\begin{array}{l}4 S E a S F x^{2}+\sqrt{3} S E b S F z^{2}-\sqrt{3} S E b S F y^{2}+S E a S F y^{2}+S E a S F z^{2} \\
-\sqrt{3}\left(-\sqrt{3} S E b S F z^{2}-\sqrt{3} S E b S F y^{2}-S E a S F z^{2}+S E a S F y^{2}\right)\end{array}$ \\
\hline
\end{tabular}

Symmetry adapted expressions are: 
(cont.)

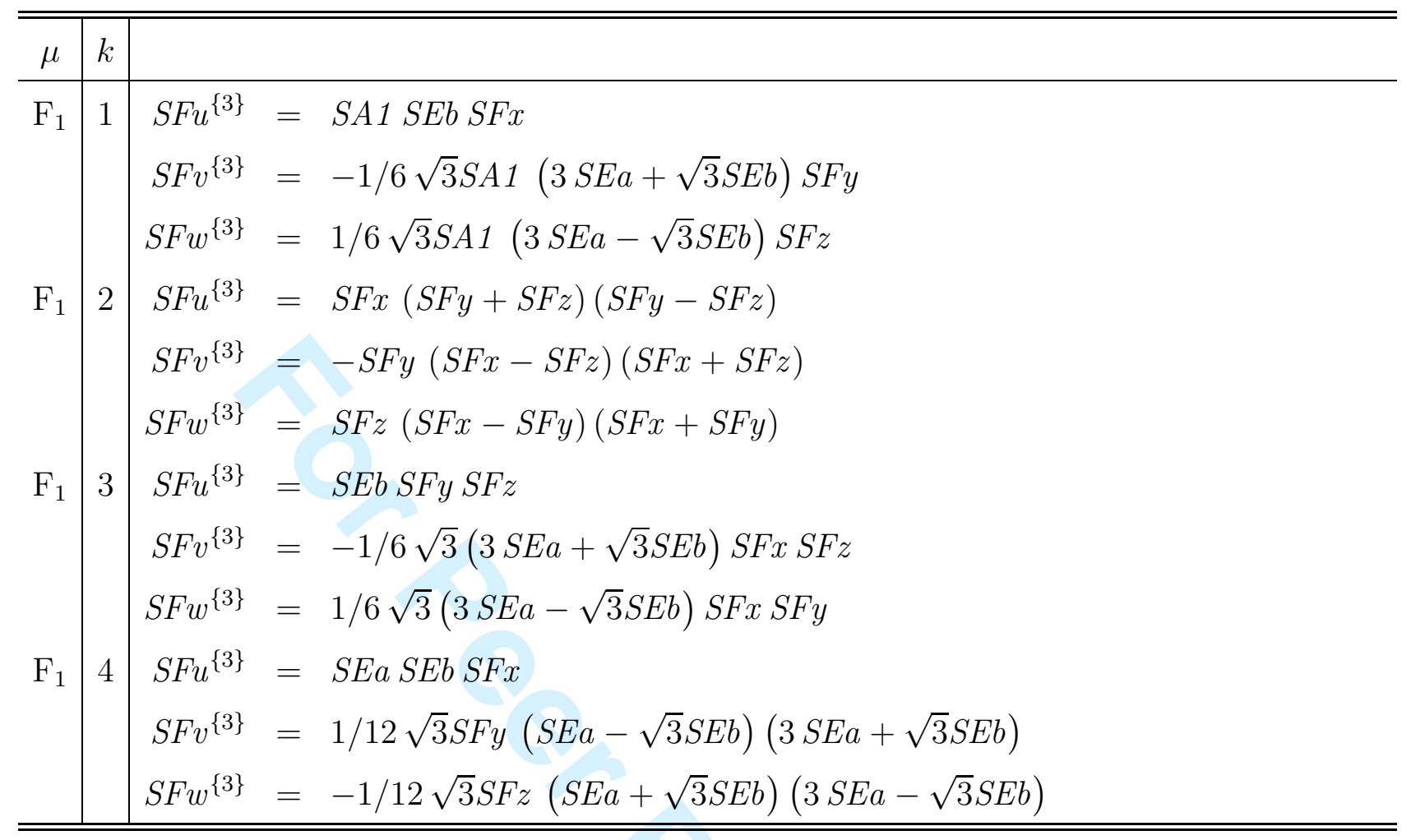

URL: http://mc.manusł \&iptcentral.com/tandf/tmph 


1
2
3
4
5
6
7
8
9
10
11
12
13
14
15
16
17
18
19
20
21
22
23
24
25
26
27
28
29
30
31
32
33
34
35
36
37
38
39
40
41
42
43
44
45
46
47
48
49
50
51
52
53
54
55
56
57
59
60

(cont.)

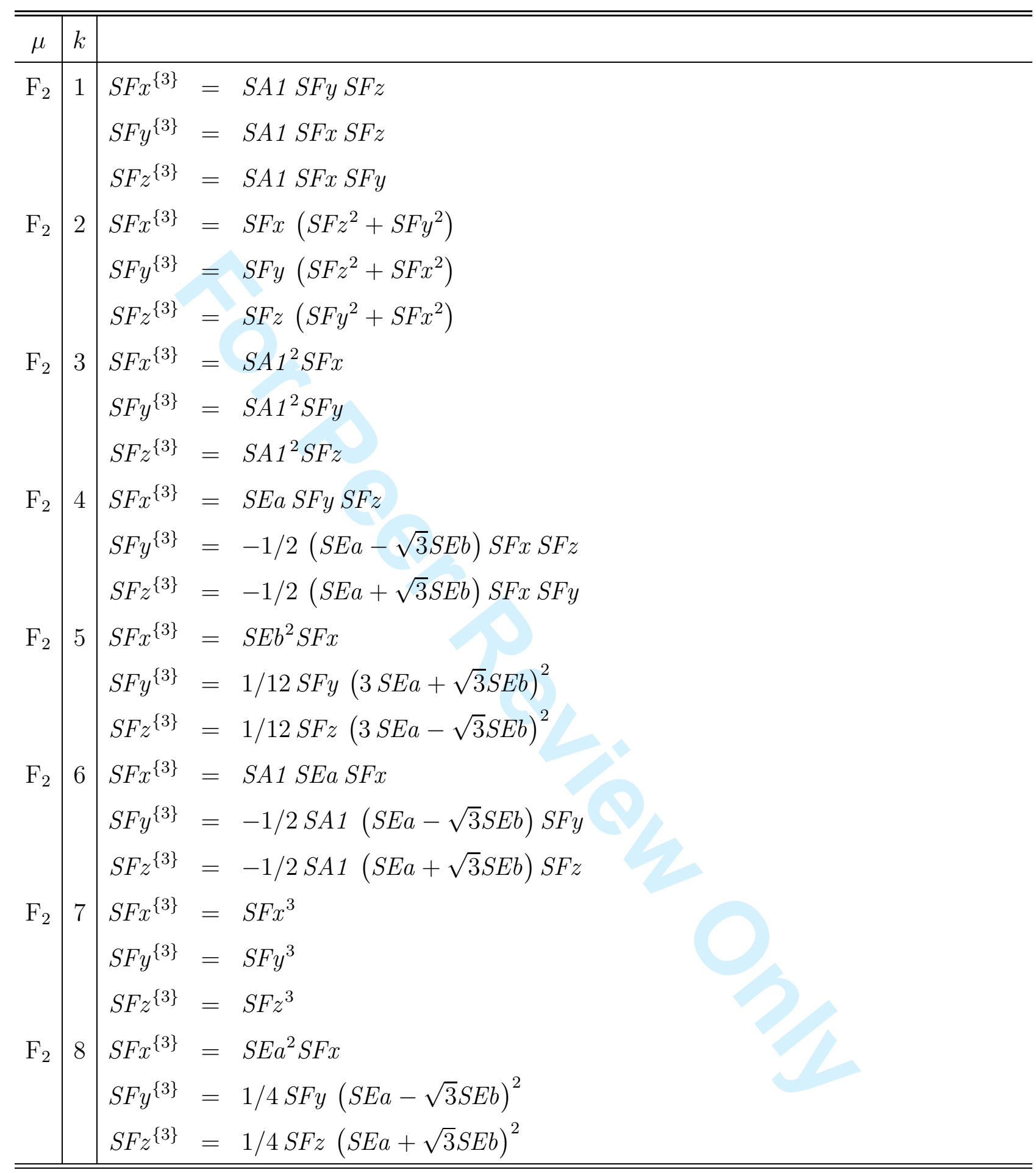

URL: http://mc.manusoliptcentral.com/tandf/tmph 


\section{B.3 Expressions of order 4}

The product representation

$$
\Gamma^{(4)}=\left(\mathcal{D}^{\left(\mathrm{A}_{1}\right)} \oplus \mathcal{D}^{(\mathrm{E})} \oplus \mathcal{D}^{\left(\mathrm{F}_{2}\right)}\right)^{\otimes 4}
$$

reduces as

$$
\Gamma^{\mathrm{red}(4)}=11 \mathcal{D}^{\left(\mathrm{A}_{1}\right)} \oplus 3 \mathcal{D}^{\left(\mathrm{A}_{2}\right)} \oplus 14 \mathcal{D}^{(\mathrm{E})} \oplus 11 \mathcal{D}^{\left(\mathrm{F}_{1}\right)} \oplus 17 \mathcal{D}^{\left(\mathrm{F}_{2}\right)}
$$

\begin{tabular}{|c|c|c|c|c|}
\hline$\mu$ & $k$ & & & \\
\hline $\mathrm{A}_{1}$ & 1 & $S A 1^{\{4\}}$ & $=$ & $S A 1^{4}$ \\
\hline $\mathrm{A}_{1}$ & 2 & $S A 1^{\{4\}}$ & $=$ & $\left(S E b^{2}+S E a^{2}\right) S A 1^{2}$ \\
\hline $\mathrm{A}_{1}$ & 3 & $S A 1^{\{4\}}$ & $=$ & $\left(S F z^{2}+S F y^{2}+S F x^{2}\right) S A 1^{2}$ \\
\hline $\mathrm{A}_{1}$ & 4 & $S A 1^{\{4\}}$ & $=$ & $S A 1 S E a\left(-3 S E b^{2}+S E a^{2}\right)$ \\
\hline $\mathrm{A}_{1}$ & 5 & $S A 1^{\{4\}}$ & $=$ & $S A 1\left(-\sqrt{3} S E b S F z^{2}+\sqrt{3} S E b S F y^{2}+2 S E a S F x^{2}-S E a S F y^{2}-S E a S F z^{2}\right)$ \\
\hline $\mathrm{A}_{1}$ & 6 & $S A 1^{\{4\}}$ & $=$ & $S A 1 S F x S F y S F z$ \\
\hline $\mathrm{A}_{1}$ & 7 & $S A 1^{\{4\}}$ & $=$ & $\left(S E b^{2}+S E a^{2}\right)^{2}$ \\
\hline $\mathrm{A}_{1}$ & 8 & $S A 1^{\{4\}}$ & $=$ & $3 S E b^{2} S F z^{2}+3 S E b^{2} S F y^{2}+S E a^{2} S F z^{2}+S E a^{2} S F y^{2}$ \\
\hline & & & & $+4 S E a^{2} S F x^{2}-2 \sqrt{3} S E a S E b S F y^{2}+2 \sqrt{3} S E a S E b S F z^{2}$ \\
\hline $\mathrm{A}_{1}$ & 9 & $S A 1^{\{4\}}$ & $=$ & $4 S E b^{2} S F x^{2}+S E b^{2} S F z^{2}+S E b^{2} S F y^{2}+3 S E a^{2} S F z^{2}$ \\
\hline & & & & $+3 S E a^{2} S F y^{2}+2 \sqrt{3} S E a S E b S F y^{2}-2 \sqrt{3} S E a S E b S F z^{2}$ \\
\hline $\mathrm{A}_{1}$ & 10 & $S A 1^{\{4\}}$ & $=$ & $S F z^{4}+S F y^{4}+S F x^{4}$ \\
\hline $\mathrm{A}_{1}$ & 11 & $S A 1^{\{4\}}$ & $=$ & $S F y^{2} S F z^{2}+S F x^{2} S F z^{2}+S F x^{2} S F y^{2}$ \\
\hline $\mathrm{A}_{2}$ & 1 & $S A \mathscr{2}^{\{4\}}$ & $=$ & $S A 1 S E b\left(3 S E a^{2}-S E b^{2}\right)$ \\
\hline $\mathrm{A}_{2}$ & 2 & $S A 2^{\{4\}}$ & $=$ & $S A 1\left(-2 \sqrt{3} S E b S F x^{2}+\sqrt{3} S E b S F y^{2}+\sqrt{3} S E b S F z^{2}\right)$ \\
\hline \multirow{3}{*}{$\mathrm{A}_{2}$} & \multirow{3}{*}{3} & \multirow{3}{*}{$S A 2^{\{4\}}$} & & $+S A 1\left(3 S E a S F y^{2}-3 S E a S F z^{2}\right)$ \\
\hline & & & $=$ & $3 S E b^{2} S F z^{2}-3 S E b^{2} S F y^{2}-3 S E a^{2} S F z^{2}+3 S E a^{2} S F y^{2}$ \\
\hline & & & & $+4 \sqrt{3} S E a S E b S F x^{2}-2 \sqrt{3} S E a S E b S F y^{2}-2 \sqrt{3} S E a S E b S F z^{2}$ \\
\hline
\end{tabular}

The symmetry adapted expressions are:

URL: http://mc.manuङ्(fiptcentral.com/tandf/tmph 


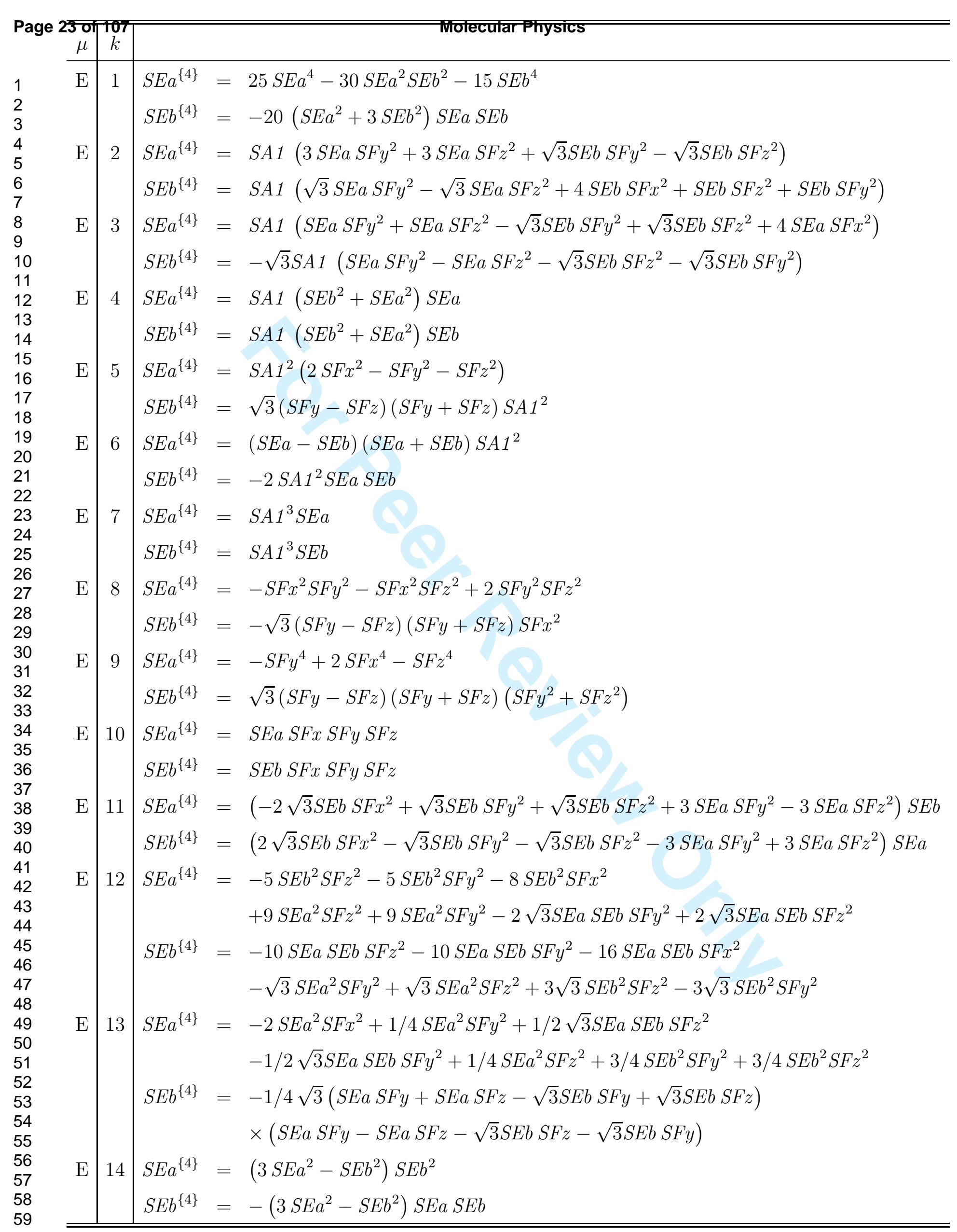


(cont.)

\begin{tabular}{|c|c|c|c|c|}
\hline$\mu$ & $k$ & & & \\
\hline \multirow[t]{3}{*}{$\mathrm{F}_{1}$} & 1 & $S F u^{\{4\}}$ & $=$ & $2 \sqrt{3} S E a^{2} S E b S F x$ \\
\hline & & $S F v^{\{4\}}$ & $=$ & $-1 / 4 S F y(3 S E a+\sqrt{3} S E b)(S E a-\sqrt{3} S E b)^{2}$ \\
\hline & & $S F w^{\{4\}}$ & $=$ & $1 / 4 S F z(3 S E a-\sqrt{3} S E b)(S E a+\sqrt{3} S E b)^{2}$ \\
\hline \multirow[t]{3}{*}{$\mathrm{F}_{1}$} & 2 & $S F u^{\{4\}}$ & $=$ & $S A 1 S F x(S F y-S F z)(S F y+S F z)$ \\
\hline & & $S F v^{\{4\}}$ & $=$ & $-S A 1(S F x-S F z)(S F x+S F z) S F y$ \\
\hline & & $S F w^{\{4\}}$ & $=$ & $S A 1(S F x-S F y)(S F x+S F y) S F z$ \\
\hline \multirow[t]{3}{*}{$\mathrm{F}_{1}$} & 3 & $S F u^{\{4\}}$ & $=$ & $2 \sqrt{6} S A 1 S E a S E b S F x$ \\
\hline & & $S F v^{\{4\}}$ & $=$ & $1 / 2 \sqrt{2} S A 1(S E a-\sqrt{3} S E b)(3 S E a+\sqrt{3} S E b) S F y$ \\
\hline & & $S F w^{\{4\}}$ & $=$ & $-1 / 2 \sqrt{2} S A 1(S E a+\sqrt{3} S E b)(3 S E a-\sqrt{3} S E b) S F z$ \\
\hline \multirow[t]{3}{*}{$\mathrm{F}_{1}$} & 4 & $S F u^{\{4\}}$ & $=$ & $2 S E b S F x^{3}$ \\
\hline & & $S F v^{\{4\}}$ & $=$ & $-1 / 3 \sqrt{3}(3 S E a+\sqrt{3} S E b) S F y^{3}$ \\
\hline & & $S F w^{\{4\}}$ & $=$ & $1 / 3 \sqrt{3}(3 S E a-\sqrt{3} S E b) S F z^{3}$ \\
\hline \multirow[t]{3}{*}{$\mathrm{F}_{1}$} & 5 & $S F u^{\{4\}}$ & $=$ & $2 S E b^{3} S F x$ \\
\hline & & $S F v^{\{4\}}$ & $=$ & $-1 / 36 \sqrt{3} S F y(3 S E a+\sqrt{3} S E b)^{3}$ \\
\hline & & $S F w^{\{4\}}$ & $=$ & $1 / 36 \sqrt{3} S F z(3 S E a-\sqrt{3} S E b)^{3}$ \\
\hline \multirow[t]{3}{*}{$\mathrm{F}_{1}$} & 6 & $S F u^{\{4\}}$ & $=$ & $S E b S F x\left(S F y^{2}+S F z^{2}\right)$ \\
\hline & & $S F v^{\{4\}}$ & $=$ & $-1 / 6 \sqrt{3}(3 S E a+\sqrt{3} S E b)\left(S F x^{2}+S F z^{2}\right) S F y$ \\
\hline & & $S F w^{\{4\}}$ & $=$ & $1 / 6 \sqrt{3}(3 S E a-\sqrt{3} S E b)\left(S F x^{2}+S F y^{2}\right) S F z$ \\
\hline \multirow[t]{3}{*}{$\mathrm{F}_{1}$} & 7 & $S F u^{\{4\}}$ & $=$ & $2 \sqrt{3} S A 1^{2} S E b S F x$ \\
\hline & & $S F v^{\{4\}}$ & $=$ & $-(3 S E a+\sqrt{3} S E b) S F y S A 1^{2}$ \\
\hline & & $S F w^{\{4\}}$ & $=$ & $S F z(3 S E a-\sqrt{3} S E b) S A 1^{2}$ \\
\hline \multirow[t]{3}{*}{$\mathrm{F}_{1}$} & 8 & $S F u^{\{4\}}$ & $=$ & $S E a S F x(S F y-S F z)(S F y+S F z)$ \\
\hline & & $S F v^{\{4\}}$ & $=$ & $1 / 2(S E a-\sqrt{3} S E b)(S F x-S F z)(S F x+S F z) S F y$ \\
\hline & & $S F w^{\{4\}}$ & $=$ & $-1 / 2(S E a+\sqrt{3} S E b)(S F x-S F y)(S F x+S F y) S F z$ \\
\hline \multirow[t]{3}{*}{$\mathrm{F}_{1}$} & 9 & $S F u^{\{4\}}$ & $=$ & $2 \sqrt{6} S E a S E b S F y S F z$ \\
\hline & & $S F v^{\{4\}}$ & $=$ & $1 / 2 \sqrt{2}(S E a-\sqrt{3} S E b)(3 S E a+\sqrt{3} S E b) S F x S F z$ \\
\hline & & $S F w^{\{4\}}$ & $=$ & $-1 / 2 \sqrt{2}(S E a+\sqrt{3} S E b)(3 S E a-\sqrt{3} S E b) S F x S F y$ \\
\hline
\end{tabular}

URL: http://mc.manusęriptcentral.com/tandf/tmph 
(cont.)

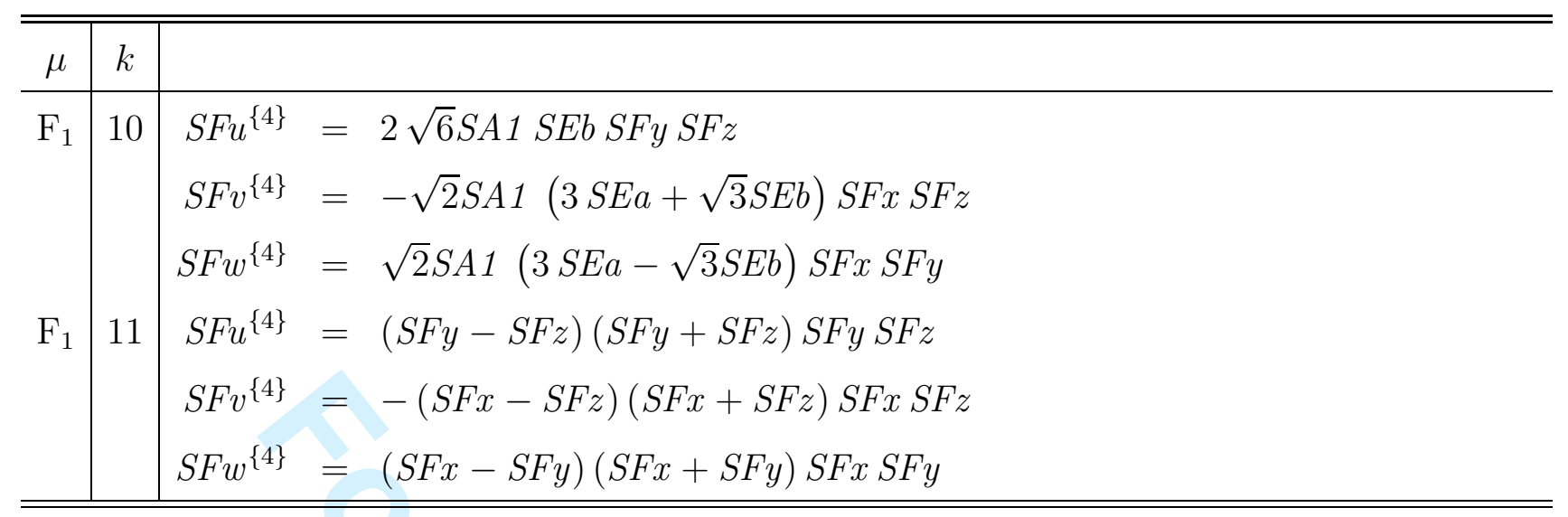

URL: http://mc.manušçiptcentral.com/tandf/tmph 
(cont.)

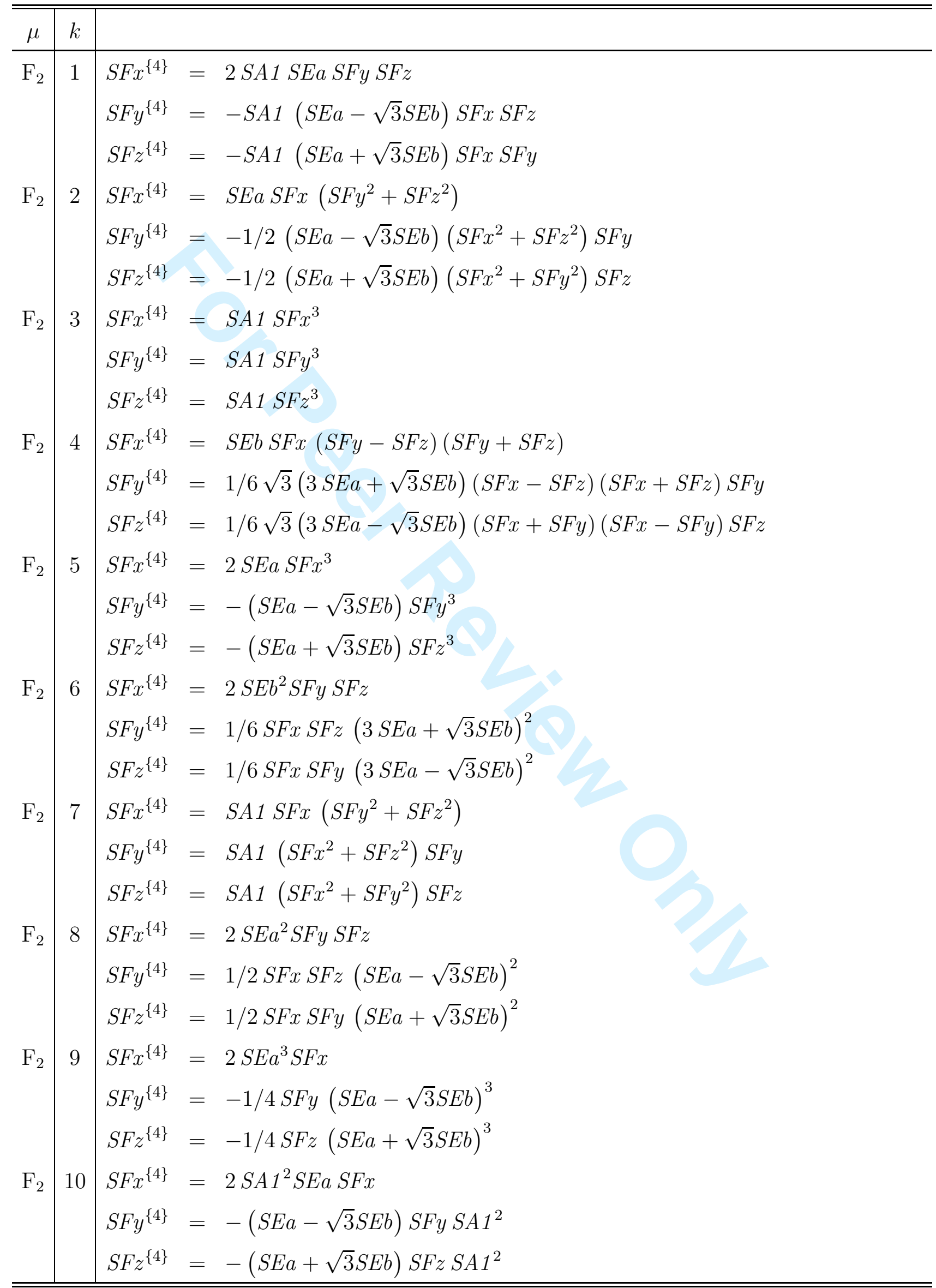


(cont.)

\begin{tabular}{|c|c|c|c|}
\hline$\mu$ & $k$ & & \\
\hline \multirow[t]{3}{*}{$\mathrm{F}_{2}$} & 11 & $S F x^{\{4\}}$ & $=2 S A 1 S E a^{2} S F x$ \\
\hline & & $S F y^{\{4\}}$ & $=1 / 2 S A 1 S F y(S E a-\sqrt{3} S E b)^{2}$ \\
\hline & & $S F z^{\{4\}}$ & $=1 / 2 S A 1 S F z(S E a+\sqrt{3} S E b)^{2}$ \\
\hline \multirow[t]{3}{*}{$\mathrm{F}_{2}$} & 12 & $S F x^{\{4\}}$ & $=2 S E a S E b^{2} S F x$ \\
\hline & & $S F y^{\{4\}}$ & $=-1 / 12(S E a-\sqrt{3} S E b) S F y(3 S E a+\sqrt{3} S E b)^{2}$ \\
\hline & & $S F z^{\{4\}}$ & $=-1 / 12(S E a+\sqrt{3} S E b) S F z(3 S E a-\sqrt{3} S E b)^{2}$ \\
\hline \multirow[t]{3}{*}{$\mathrm{F}_{2}$} & 13 & $S F x^{\{4\}}$ & $=\left(S F y^{2}+S F z^{2}\right) S F y S F z$ \\
\hline & & $S F y^{\{4\}}$ & $=\left(S F x^{2}+S F z^{2}\right) S F x S F z$ \\
\hline & & $S F z^{\{4\}}$ & $=\left(S F x^{2}+S F y^{2}\right) S F x S F y$ \\
\hline \multirow[t]{3}{*}{$\mathrm{F}_{2}$} & 14 & $S F x^{\{4\}}$ & $=S A 1^{2} S F y S F z$ \\
\hline & & $S F y^{\{4\}}$ & $=S A 1^{2} S F x S F z$ \\
\hline & & $S F z^{\{4\}}$ & $=S A 1^{2} S F x S F y$ \\
\hline \multirow[t]{3}{*}{$\mathrm{F}_{2}$} & 15 & $S F x^{\{4\}}$ & $=S F x^{2} S F y S F z$ \\
\hline & & $S F y^{\{4\}}$ & $=S F x S F y^{2} S F z$ \\
\hline & & $S F z^{\{4\}}$ & $=S F x S F y S F z^{2}$ \\
\hline \multirow[t]{3}{*}{$\mathrm{F}_{2}$} & 16 & $S F x^{\{4\}}$ & $=S A 1^{3} S F x$ \\
\hline & & $S F y^{\{4\}}$ & $=S A 1^{3} S F y$ \\
\hline & & $S F z^{\{4\}}$ & $=S A 1^{3} S F z$ \\
\hline \multirow[t]{3}{*}{$\mathrm{F}_{2}$} & 17 & $S F x^{\{4\}}$ & $=2 S A 1 S E b^{2} S F x$ \\
\hline & & $S F y^{\{4\}}$ & $=1 / 6 S A 1 S F y(3 S E a+\sqrt{3} S E b)^{2}$ \\
\hline & & $S F z^{\{4\}}$ & $=1 / 6 S A 1 S F z(3 S E a-\sqrt{3} S E b)^{2}$ \\
\hline
\end{tabular}

URL: http://mc.manušçiptcentral.com/tandf/tmph 


\section{Table captions}

Table 1. Reduction of product representations $\Gamma^{(m)}=\left(\mathcal{D}^{\left(\mathrm{A}_{1}\right)} \oplus \mathcal{D}^{(\mathrm{E})}\right)^{\otimes m}$ of the three-dimensional representation $\mathcal{D}^{\left(\mathrm{A}_{1}\right)} \oplus \mathcal{D}^{(\mathrm{E})}$ of $\mathbf{C}_{3 \mathrm{v}}$ (Eq. (12) to Eq. (13)) (space of bending coordinates in ammonia). The reduced dimension at order $m$ is the dimension of the $\mathrm{A}_{1}$-subspace in $\mathbf{S}_{\mathrm{m}}$.

Table 2. Reduction of product representations $\Gamma^{(m)}=\left(\mathcal{D}^{\left(\mathrm{A}_{1}\right)} \oplus \mathcal{D}^{(\mathrm{E})} \oplus \mathcal{D}^{\left(\mathrm{F}_{2}\right)}\right)^{\otimes m}$ of the sixdimensional representation $\left(\mathcal{D}^{\left(\mathrm{A}_{1}\right)} \oplus \mathcal{D}^{(\mathrm{E})} \oplus \mathcal{D}^{\left(\mathrm{F}_{2}\right)}\right)$ of $\mathbf{T}_{\mathrm{d}}$ (Eq. (15) to Eq. (17)) (space of bending coordinates in methane). The reduced dimension at order $m$ is the dimension of the $A_{1}$-subspace of $\mathbf{S}_{\mathrm{m}}$. 
Table 1

Marquardt and Sagui

\begin{tabular}{c|c|c|c}
\hline \hline order $(m)$ & dimension $\left(n^{m}\right)$ & reduced dimension & reduction in $\mathbf{C}_{3 \mathrm{v}}$ \\
\hline 2 & 9 & 6 & $2 \mathcal{D}^{\left(\mathrm{A}_{1}\right)} \oplus 2 \mathcal{D}^{(\mathrm{E})}$ \\
3 & 27 & 10 & $3 \mathcal{D}^{\left(\mathrm{A}_{1}\right)} \oplus \mathcal{D}^{\left(\mathrm{A}_{2}\right)} \oplus 3 \mathcal{D}^{(\mathrm{E})}$ \\
4 & 81 & 15 & $4 \mathcal{D}^{\left(\mathrm{A}_{1}\right)} \oplus 1 \mathcal{D}^{\left(\mathrm{A}_{2}\right)} \oplus 5 \mathcal{D}^{(\mathrm{E})}$ \\
\hline \hline
\end{tabular}


Table 2

Marquardt and Sagui

\begin{tabular}{c|c|c|c}
\hline \hline order $(m)$ & dimension $\left(n^{m}\right)$ & reduced dimension & reduction in $\mathbf{T}_{\mathrm{d}}$ \\
\hline 2 & 36 & 21 & $3 \mathcal{D}^{\left(\mathrm{A}_{1}\right)} \oplus 3 \mathcal{D}^{(\mathrm{E})} \oplus 1 \mathcal{D}^{\left(\mathrm{F}_{1}\right)} \oplus 3 \mathcal{D}^{\left(\mathrm{F}_{2}\right)}$ \\
3 & 216 & 56 & $6 \mathcal{D}^{\left(\mathrm{A}_{1}\right)} \oplus 2 \mathcal{D}^{\left(\mathrm{A}_{2}\right)} \oplus 6 \mathcal{D}^{(\mathrm{E})} \oplus 4 \mathcal{D}^{\left(\mathrm{F}_{1}\right)} \oplus 8 \mathcal{D}^{\left(\mathrm{F}_{2}\right)}$ \\
4 & 1296 & 126 & $11 \mathcal{D}^{\left(\mathrm{A}_{1}\right)} \oplus 3 \mathcal{D}^{\left(\mathrm{A}_{2}\right)} \oplus 14 \mathcal{D}^{(\mathrm{E})} \oplus 11 \mathcal{D}^{\left(\mathrm{F}_{1}\right)} \oplus 17 \mathcal{D}^{\left(\mathrm{F}_{2}\right)}$ \\
\hline \hline
\end{tabular}




\section{REDTEN: A symbolic algebra program for the automatic reduction of powers of representations of finite groups}

\author{
Roberto Marquardt* and Kenneth Sagui \\ Laboratoire de Chimie Theorique, Université de Marne-la-Vallée \\ 5 Bd Descartes (Champs-sur-Marne), F-77454 Marne-la-Vallée CEDEX 2, France
}

\section{USER GUIDE and MAPLE code REDTEN}

prepared as Supplementary Publication

to be deposited with the British Library Document Supply Centre

\footnotetext{
* present address (21/01/2007) and email: roberto.marquardt@chimie.u-strasbg.fr correspondence and proofs to Prof. Roberto Marquardt

Laboratoire de Chimie Quantique - Institut de Chimie - LC3 UMR 7177 CNRS/ULP

Université Louis Pasteur - 4, rue Blaise Pascal - 67000 STRASBOURG - France

Fax 0(033)390241589
} 


\section{Contents}

1 Introduction $\quad 1$

2 Name $\quad 1$

3 Distribution list (version 060527) 1

4 Installation 2

5 Input 2

$6 \quad$ Running the calculation $\quad 4$

7 Output files $\quad 5$

8 Brief description of different subroutines used in the algorithm $\quad 6$

$9 \begin{array}{ll}\text { Character tables } & 7\end{array}$

$\begin{array}{ll}\text { A main.cmd } & 9\end{array}$

$\begin{array}{lr}\text { B turm.cmd } & 21\end{array}$

$\begin{array}{ll}\mathrm{C} \text { redtenlib.cmd } & 25\end{array}$

$\begin{array}{ll}\text { D sample input } & 69\end{array}$

$\begin{array}{ll}\text { E sample output } & 70\end{array}$

$\begin{array}{ll}\text { F unix script } & 75\end{array}$ 


\section{Introduction}

This code implements an algorithm to perform the reduction of representations in general, and the symmetry adaption for power representations of some finite groups in particular, according to the procedure described in the article "A complete list of symmetry adapted expressions to the fourth power for compact bending potentials in molecules with $\mathbf{C}_{3 \mathrm{v}}$ and $\mathbf{T}_{\mathrm{d}}$ symmetry from a general symbolic algebra program" [1] that is related with it.

The implementation of the code is based on the MAPLE program package for symbolic algebra. The present version (060527) runs under MAPLE 10 release [2].

This document contains information on the distribution of the code and instructions for installation and use. A hard copy of the code related to the distribution list described below is given in the appendices.

\section{Name}

The code has the name REDTEN.

\section{$3 \quad$ Distribution list (version 060527)}

A distribution package is available from the corresponding author at the address given in the cover page. This package contains the following files:

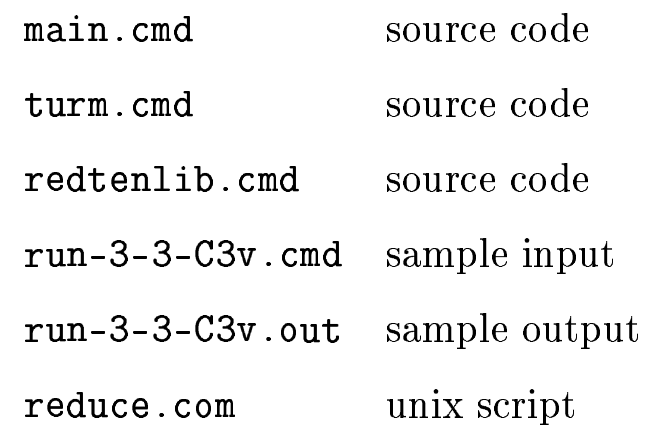

All files are open source. The code may be changed or appended by interested users when they make a reference to the original article [1]. 


\section{Installation}

The package is delivered as a zip file. Unpacking yields a directory, named package, and a pdf file containing the present document. To run the code, enter the directory package, or any other working directory, into which the contents of package have been copied.

Give the unix script reduce.com "execute permission", then run it from the working directory (see section 6 below).

\section{Input}

Input files must be named according to the following nomenclature:

run-M-NDIM1-GROUP.cmd

where $M$ is a positive integer that gives the order of the tensor space, NDIM1 is a positive integer that gives the dimension of the first order linear space $V$ and $G R O U P$ is a code that defines the finite symmetry group to be considered.

Currently, accepted codes are: $G R O U P \in\{\mathrm{Cs}, \mathrm{C} 2 \mathrm{v}, \mathrm{C} 3 \mathrm{v}, \mathrm{Td}\}$ (with size sensitive letters). The input file must contain the following cards:

$\mathbf{M}:=$ the order of the tensor space $V^{\otimes m}$

GROUP:= the code for the finite symmetry group

NDIM1:= the dimension of the first order vector space $V$

MUE1:= $\left[\mu_{1}, \ldots, \mu_{N}\right]$, MAPLE-table of $N$ irreducible representations of group GROUP which are used to build up the $V$ space; $\mu_{j}$ is the number of the $j$-th irreducible representation in the corresponding character table (see section 9 below for a list of character tables used in the code); of course, $\sum_{n=1}^{N} d_{\mu_{n}} \cdot \gamma_{\mu_{n}}=N D I M 1$ must 
hold, where $d_{\mu_{n}}$ is the dimension and $\gamma_{\mu_{n}}$ is a possible multiplicity of the irreducible representation species $\mu_{n}$ in $V$ (see next card)

MMU1:= $\left[\gamma_{1}, \ldots, \gamma_{N}\right]$, MAPLE-table of multiplicities of each irreducible representation species used to build up the first order vector space $V$

V1I[1][1]:= MAPLE-table of symbols to represent the components of the first irreducible vector of species 1 (the table has $d_{1}$ entries)

$\vdots$

V1I[1] $\left.\gamma_{1}\right]:=$ MAPLE-table of symbols to represent the components of the irreducible vector number $\gamma_{1}$ of species 1 (the table has $d_{1}$ entries)

$\vdots$

$\vdots$

$\mathrm{V} 1 \mathrm{I}[\mu][1]:=$ MAPLE-table of symbols to represent the components of the first irreducible vector of species $\mu$ (the table has $d_{\mu}$ entries)

$\vdots$

$\operatorname{V1I}[\mu]\left[\gamma_{\mu}\right]:=$ MAPLE-table of symbols to represent the components of the irreducible vector number $\gamma_{\mu}$ of species $\mu$ (the table has $d_{\mu}$ entries)

read "turm.cmd": Call of routine turm.cmd which is designed to get the representation of the symmetric Group $\mathbf{S}_{\mathrm{m}}$ in tensor space $V^{\otimes m}$ and the reduction thereof in $\mathbf{S}_{\mathrm{m}}$; this routine essentially calculates the rectangular transformation matrix $\boldsymbol{T}^{\left(N D I M 1^{M} ; \gamma\right)}$ from equation (13) of the article, where $\gamma$ is the multiplicity of the $A_{1}\left(\mathbf{S}_{M}\right)$-species; when this routine is run for the first time, an intermediate output file is generated that is read in the subsequent calls of the program, for the same value of $M$; this card can therefore be commented in subsequent calls by inserting the symbol $\sharp$ in the first column of the card 
read "main.cmd": Call of routine main. cmd which concentrates all the stages of the algorithm, as indicated by its name.

To illustrate this, we give the example of the input file designed to run a calculation of the reduction of direct product representations of $\mathbf{C}_{3 \mathrm{v}}$ of order 3 for a three-dimensional first-order vector, as showed in the article that goes with this program [1].

First, the input file in question must be named run-3-3-C3v .cmd.

Secondly, it must be presented in the form given in Figure 1.

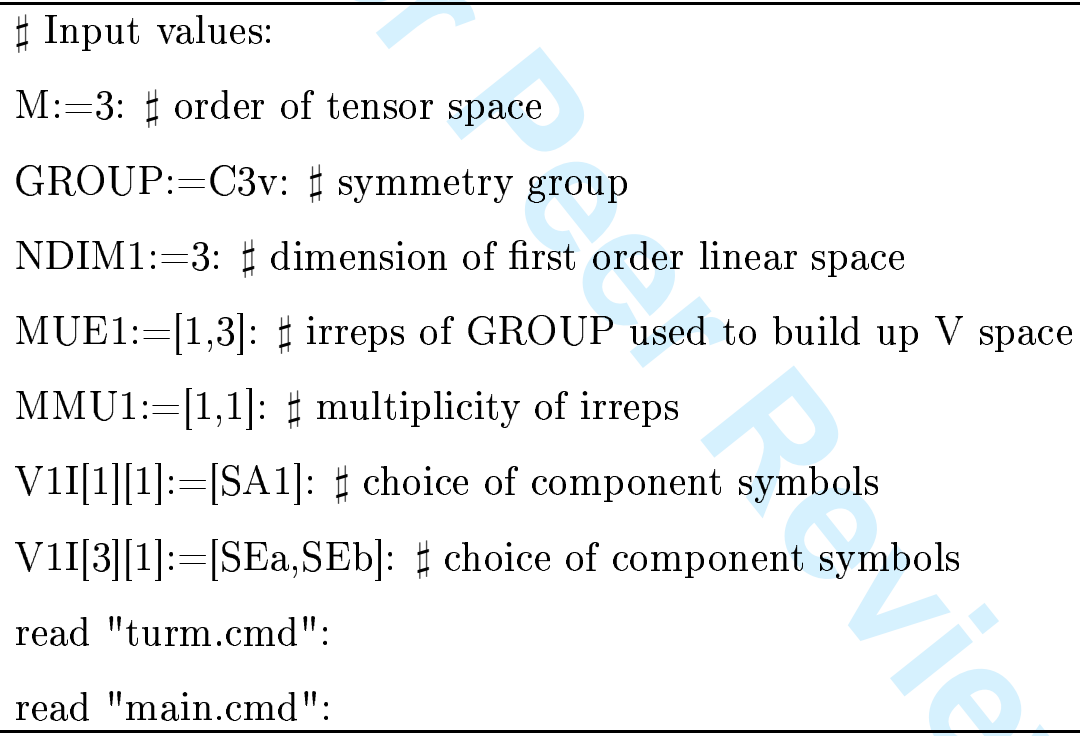

Figure 1: Example of an input file : run-3-3-C3v.cmd; the symbol $\sharp$ is followed of a comment in the maple language.

\section{Running the calculation}

The program can be launched by using the unix script reduce.com as follows:

\section{reduce.com $M \quad N D I M 1 \quad G R O U P$}

The three arguments have the same meaning as for the naming of the files. The script opens MAPLE, runs the code, writes output files and closes MAPLE automatically. The 
program may be run as a background job. For the example given above, type for instance reduce.com 33 C3v\&.

\section{Output files}

Several output files are generated:

turmdata-M-NDIM1.res

Data file in MAPLE language to be used in routine main.cmd, containing the rectangular transformation matrix $\boldsymbol{T}^{\left(N D I M 1^{M} ; \gamma\right)}$.

turm-M-NDIM1.out

Logfile for routine turm. cmd.

REPMDATA-M-NDIM1-GROUP.res

Data file in MAPLE language containing the representation matrices $\boldsymbol{\Gamma}^{\left(N D I M 1^{M}\right)}$ obtained from equation (11) of the article.

\section{GAMMAMr $-M-N D I M 1-G R O U P$.res}

Data file in MAPLE language containing the reduced representations $\Gamma^{(\gamma)}$ obtained from equation (14).

\section{run-M-NDIM1-GROUP.res}

File in MAPLE language containing the following data, in the given order:

NIRRP, the number of irreducible representions;

REDF, MAPLE-table with NIRRP entries containing the reduction factors (multiplicities) $\gamma_{\mu}$ into the irreductible representations $\mu$;

DIRRP, MAPLE-table with NIRRP entries containing the dimensions $d_{\mu}$ of the irreducible representations;

GLABE, MAPLE-table with NIRRP entries containing labels for the irreducible representations;

Vms, MAPLE-table containing entries $\operatorname{Vms}[\mu]$; only irreducible representations $\mu$ for which $\gamma_{\mu}>0$ are given; each entry is a vector of length $\gamma_{\mu} \times d_{\mu}$ and contains symmetry adapted expressions; 
run-M-NDIM1-GROUP . out

Logfile of routine main. cmd containing self-explanatory output.

\section{Brief description of different subroutines used in the algorithm}

In main.cmd the program library redtenlib.cmd is loaded which gathers all the subroutines used in the algorithm with the exception of turm.cmd. In what follows, the function of each routine will be briefly described.

prtime displays the duration of each stage of the calculation

irrep definition of irreducible representations

defk definition of tensor space key

repm calculation of group representation matrices in power $M$ tensor space

simtra calculation of similarity transformation

Sm calculation of permutation matrices (regular representation of the symmetry group $\left.\mathbf{S}_{\mathrm{M}}\right)$

SmInVm calculation of representation matrices $\boldsymbol{\Sigma}^{\left(N D I M 1^{M}\right)}$

redA1 calculation of symmetry reduction by projection on $A_{1}$ irreducible representation vector subspace using Wigner's projection operator [3]

redfac calculation of symmetry reduction factors

reduce calculation of symmetry reduction using generalized projection operators as given by Hamermesh $[4,5]$

orthonorm calculates a set of ortho-normalized vectors following the Gram-Schmidt procedure [6]

vecnorm calculation of a vector norm. 


\section{Character tables}

We used the following conventions for irreducible representations:

\begin{tabular}{l|l|l|rr}
\hline \hline $\mathrm{C}_{\mathrm{s}}$ & $\mu$ & label & $E$ & $\sigma$ \\
\hline & 1 & $\mathrm{~A}$ & 1 & 1 \\
& 2 & $\mathrm{~B}$ & 1 & -1 \\
\hline \hline
\end{tabular}

\begin{tabular}{r|l|l|rrrr}
\hline \hline $\mathrm{C}_{2 \mathrm{v}}$ & $\mu$ & label & $E$ & $C_{2}$ & $\sigma_{\mathrm{v}}$ & $\sigma_{\mathrm{h}}$ \\
\hline & 1 & $\mathrm{~A}_{1}$ & 1 & 1 & 1 & 1 \\
& 2 & $\mathrm{~A}_{2}$ & 1 & 1 & -1 & -1 \\
& 3 & $\mathrm{~B}_{1}$ & 1 & -1 & -1 & 1 \\
& 4 & $\mathrm{~B}_{2}$ & 1 & -1 & 1 & -1 \\
\hline \hline
\end{tabular}

\begin{tabular}{l|l|l|rrr}
\hline \hline $\mathbf{C}_{3 \mathrm{v}}$ & $\mu$ & label & $E$ & $2 C_{3}$ & $3 \sigma_{\mathrm{v}}$ \\
\hline & 1 & $\mathrm{~A}_{1}$ & 1 & 1 & 1 \\
& 2 & $\mathrm{~A}_{2}$ & 1 & 1 & -1 \\
& 3 & $\mathrm{E}$ & 2 & -1 & 0 \\
\hline \hline
\end{tabular}

\begin{tabular}{r|l|l|rrrrr}
\hline \hline $\mathbf{T}_{\mathrm{d}}$ & $\mu$ & label & $E$ & $6 C_{2}^{\prime}$ & $8 C_{3}$ & $6 C_{4}$ & $3 C_{2}$ \\
\hline & 1 & $\mathrm{~A}_{1}$ & 1 & 1 & 1 & 1 & 1 \\
& 2 & $\mathrm{~A}_{2}$ & 1 & -1 & 1 & -1 & 1 \\
& 3 & $\mathrm{E}$ & 2 & 0 & -1 & 0 & 2 \\
& 4 & $\mathrm{~F}_{1}$ & 3 & -1 & 0 & 1 & -1 \\
& 5 & $\mathrm{~F}_{2}$ & 3 & 1 & 0 & -1 & -1 \\
\hline \hline
\end{tabular}




\section{References}

[1] Roberto Marquardt and Kenneth Sagui. A complete list of symmetry adapted expressions to the fourth power for compact bending potentials in molecules with $\mathbf{C}_{3 \mathrm{v}}$ and $\mathbf{T}_{\mathrm{d}}$ symmetry from a general symbolic algebra program, 2007. Submitted to Molecular Physics.

[2] Maple V Release 10. Copyright (c) 2004-2005 by Maplesoft, a division of Waterloo Maple Software Inc.

[3] E. Wigner. Group Theory. Academic Press, New York, 1959.

[4] M. Hamermesh. Group Theory and Its Application to Physical Problems. AddisonWesley, Reading, Massachusetts, 1962.

[5] J. P. Elliot and P. G. Dawber. Symmetry in Physics. Oxford University Press, New York, 1979.

[6] H.-J. Kowalsky. Lineare Algebra. de Gruyter, Berlin, New York, 1979. 


\section{A main.cmd}

\# This file is the program REDTEN. It runs an automatic reduction of a tensor product

\# representation of order $\mathrm{m}$.

\#

\# Authors: R. Marquardt and K. Sagui

\# Université de Marne-la-Vallée

\# 5 Bd Descartes, 77454 Marne-la-Vallée CEDEX 2

\# email for contact: roberto.marquardt@univ-mlv.fr

\#

— \# Version REDTEN-060527

$\#$

\# Needed programs: MAPLE 9.5 or MAPLE 10

\#

\# Input parameters M (order of tensor space),

\# GROUP (symmetry group label),

\# NDIM1 (dimension of first order linear space for check),

\# MUE1 (list of irreps of GROUP used to build up V1),

\# MMU1 (list of multiplicities of irreps),

\# V1I[MUE1[mu]][MMU1[mu]] (list of component symbols for irrep MUE1[mu])

\# must be set in calling program. 
\#

restart:

time $0:=$ time () :

\# 1) Load subroutines and libraries:

read 'redtenlib.cmd':

with (linalg):

with(LinearAlgebra):

outputfilename:=cat ("run-" , M, "-" , NDIM1, "-" , GROUP, " . out") :

outputfile:=fopen (outputfilename, WRITE) :

\# writeto(outputfile):

• \# interface (quiet=true):

\#

prtime(outputfile,1,"loading ", time0):

\# 2) Load information on irreductible representations of the symmetry groups. GDATA : =irrep (GROUP) :

\# Procedure irrep returns a list of 6 items:

NORDR:=GDATA [1]: \# the group order .

NIRRP:=GDATA[2]: \# the number of irreductible representations of the group.

GCHAR:=GDATA [3]: \# a NIRRPxNORDR matrix containing the character table.

GLABE:=GDATA [4]: \# a 1..NIRRP array containing usual labels of the irr. rep.

GIRRP:=GDATA [5]: \# a nested list of matrices of the form GIRRP $[i][n][k, j]$, 
\# where $i$ is the number of the irreductible representation,

\# $\mathrm{n}$ is the number of the operation,

\# $\quad \mathrm{k}$ and $\mathrm{j}$ are indices from 1..NDIM(i),

\# where $\operatorname{NDIM}(i)=\operatorname{GCHAR}[i, 1]$.

NBG:=GDATA [6]: \# number of any operation with nondiagonal

\# degenerate irreductible representation; set to 1 if none prtime (outputfile,2, "read irreps",time0):

\#

\# 3) Build up 1st order vector space and group representations:

for mu from 1 to NIRRP do

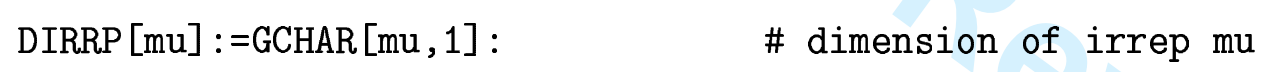


for id from 1 to DIRRP [MUE1 [mu]] do mdim:=mdim+1 :

od :

di : =nops (V1I [MUE1 [mu] ] [im]) :

if ( $\mathrm{di}<>\operatorname{DIRRP}[\mathrm{MUE} 1[\mathrm{mu}]])$ then

error "Mismatch with dimensions of V1I on input. Program interrupted."

end if:

V1I $[$ MUE1 $[\mathrm{mu}]][\mathrm{im}]:=\operatorname{convert}(\mathrm{V} 1 \mathrm{I}[\mathrm{MUE} 1[\mathrm{mu}]][\mathrm{im}]$, vector) :

od:

od:

$\stackrel{N}{N} \quad$ if (mdim $<>$ NDIM1) then

error "Mismatch with NDIM1 on input. Program interrupted."

end if:

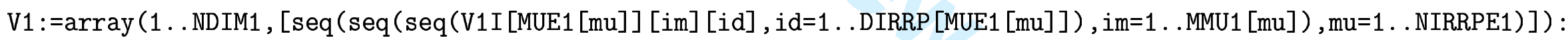
for $\mathrm{n}$ from 1 to NORDR do

GAMMA1 [n] :=matrix (NDIM1, NDIM1) :

GAMMA1 [n] : =diag ( $\operatorname{seq}(\operatorname{seq}(\operatorname{eval}(\operatorname{GIRRP}[M U E 1[\mathrm{mu}]][\mathrm{n}]), \mathrm{im}=1 \ldots$.MMU1 [mu] $), \mathrm{mu}=1 \ldots$ NIRRPE1) $)$ :

od:

prtime(outputfile, 3 , "setup GAMMA1",time0):

\# 4) Define tensor space and key: 
KDATA $:=\operatorname{defk}(M, N D I M 1):$

\# Procedure defk returns 2 items:

NDIMm:=KDATA[1]: \# tensor space dimension

KEY:=KDATA[2]: \# matrix MxNDIMm defining the tensor space.

$\mathrm{Vm}:=\operatorname{array}(1 \ldots \mathrm{NDIMm})$ :

for nd from 1 to NDIMm do \# definition of tensor space

$\operatorname{Vm}[$ nd $]:=1$ :

for $\mathrm{mm}$ from 1 to $M$ do

$\mathrm{Vm}[\mathrm{nd}]:=\mathrm{Vm}[\mathrm{nd}] * \mathrm{~V} 1[\mathrm{KEY}[\mathrm{mm}, \mathrm{nd}]]:$

od:

œu od:

prtime(outputfile,4,"define tensor space key",time0):

\# 5) Build up order $m$ vector space and group representations: GAMMAm : =array $(1$. NORDR) :

for $\mathrm{n}$ from 1 to NORDR do

GAMMAm $[\mathrm{n}]:=\operatorname{repm}($ M , NDIMm, KEY , GAMMA1 $[\mathrm{n}])$ :

nout : $=500+n$ :

prtime(outputfile,nout, "setup tensor space, operation ",time0):

\# Procedure repm returns 1 item: the representation matrix NDIMmxNDIMm od:

datfile:=cat ("REPMDATA -" , M, " - ", NDIM1, " - ", GROUP, ".res") : 
save GAMMAm, datfile:

\# read datfile:

prtime(outputfile,5, "setup tensor space",time0):

\#

\# 6) Reduce tensor space Vm into A1 space of Sm by similarity transformation. turmdatafile:=cat ("turmdata-", M, "-" , NDIM1, ".res") :

read turmdatafile:

\# turmdatafile returns REDSM, a list of 5 items:

MM:=REDSM[1]: \# the order, must match M.

MDIM1:=REDSM[2]: \# dimension of 1st order space, must match NDIM1.

- MIRRP:=REDSM[3]: \# number of irreductible representations (irreps) of Sm.

GAMMS:=REDSM [4]: \# list 1..MIRRP of reduction factors

\# of $\mathrm{Sm}$ representation in $\mathrm{Vm}$.

TS:=REDSM[5]: \# NDIMmXGAMMS[1] transformation matrix into A1 space.

if $(\mathrm{MM}<>\mathrm{M})$ then

error "Order mismatch with data on file",turmdatafile

end if :

if (MDIM1 $<>$ NDIM1) then

error "NDIM1 mismatch with data on file",turmdatafile

end if:

NDIMmr:=GAMMS[1]: \# Reduced dimension of $\mathrm{Vm}$; only $\mathrm{A} 1$ species of $\mathrm{Sm}$ is kept 
TSr : =matrix (NDIMm, NDIMmr) :

TSr:=submatrix (TS, 1..NDIMm,1..NDIMmr): \# get reduction trafo

TSrT : =transpose (TSr) :

GAMMAmr : =array (1. NORDR) :

for $\mathrm{n}$ from 1 to NORDR do

GAMMAmr $[\mathrm{n}]:=\operatorname{simtra}(\mathrm{TSr}, \mathrm{TSrT}, \mathrm{GAMMAm}[\mathrm{n}])$ : \# Procedure simtra returns TT*G*T

nout : $=600+\mathrm{n}$ :

prtime(outputfile, nout, "reduction in Sm, operation ",time0):

od:

\# GAMMAmr : =eval (GAMMAmr, 4$)$ :

datfile:=cat ("GAMMAmr-" , M , " - ", NDIM1 , " - ", GROUP, " .res"):

save GAMMAmr, datfile:

\# read datfile:

Vmr : =array (1 . NDIMmr) :

Vmr:=multiply (TSrT, Vm): \# Vector components in A1 space of $\mathrm{Sm}$.

TT : =matrix (NDIMmr, NDIMm) :

TT:=evalm(TSrT): \# backup final transformation of $\mathrm{Vm}$

prtime(outputfile, 6, "reduction in Sm", time0):

\# 7) Get reduction factors of GAMMAmr in GROUP

REDF : =redfac (NORDR , NIRRP , GCHAR, GLABE , NDIMmr , GAMMAmr) :

\# Procedure redfac returns 1 item: a list 1 .. NIRRP of reduction factors 
prtime (outputfile, 7,"reduction factors",time0):

\# 8) Reduce GAMMAmr irrep by irrep for mu from 1 to NIRRP do

gammu : $=\operatorname{REDF}[\mathrm{mu}]$ :

$\mathrm{dmu}:=\operatorname{DIRRP}[\mathrm{mu}]$ :

$\mathrm{nmu}:=$ gammu $* \mathrm{dmu}$ :

if (gammu > 0) then

$\operatorname{Ti}[\mathrm{mu}]:=\operatorname{matrix}(\mathrm{NDIMmr}, \mathrm{nmu})$ :

Ti [mu] : =reduce (mu , gammu , NORDR , GIRRP , GCHAR , NDIMmr , GAMMAmr , time0):

\# Procedure reduce returns a NDIMmrx (gammu*nmu) matrix of orthogonal vectors

Б \# $\quad$ print $(\mathrm{Ti}[\mathrm{mu}])$ :

$\operatorname{TiT}[\mathrm{mu}]:=\operatorname{transpose}(\mathrm{Ti}[\mathrm{mu}])$ :

end if:

od :

prtime(outputfile, 8, "reduce GAMMAmr",time0):

\# 9) Tensor transformation

for $\mathrm{mu}$ from 1 to NIRRP do

gammu : $=$ REDF $[\mathrm{mu}]$ :

$\mathrm{dmu}:=\operatorname{DIRRP}[\mathrm{mu}]$ :

if (gammu > 0) then

Vms $[\mathrm{mu}]:=\operatorname{evalm}(\mathrm{TiT}[\mathrm{mu}] \& * \mathrm{Vmr})$ : 


\section{Page 49 of 107}




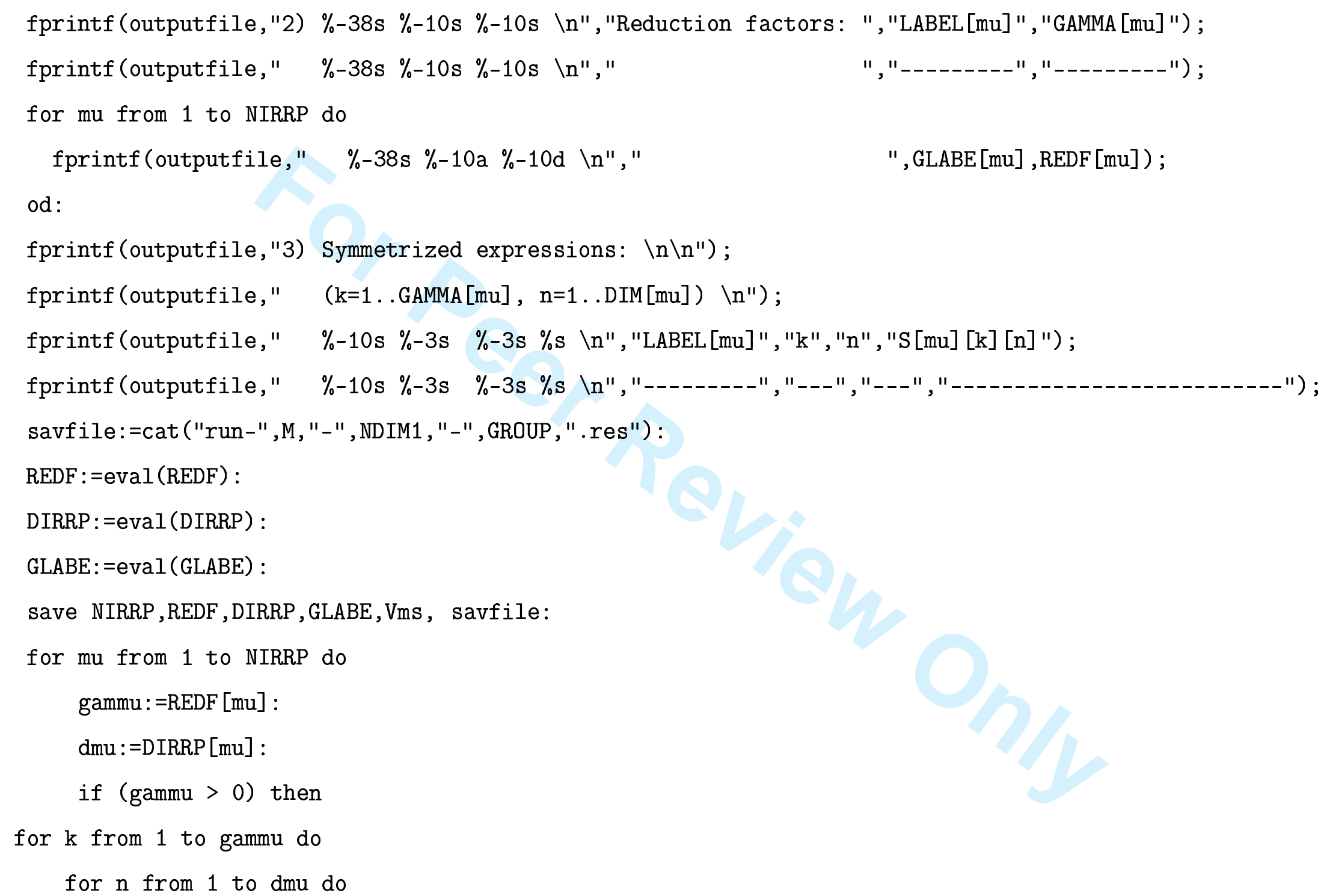


od:

od:

end if:

od :

prtime (outputfile, 11, "**************",time0) :

$\operatorname{gc}()$ :

fclose (outputfilename):

quit 


\section{B turm.cmd}

\# This file is the routine turm.cmd of program REDTEN. This routine gets

\# the representation of the symmetric Group $\mathrm{Sm}$ in tensor space $\mathrm{Vm}=\mathrm{V} * * \mathrm{~m}$,

\# and the reduction thereof in $\mathrm{Sm}$.

\#

\# Authors: R. Marquardt and K. Sagui

\# Université de Marne-la-Vallée

\# 5 Bd Descartes, 77454 Marne-la-Vallée CEDEX 2

\# email for contact: roberto.marquardt@univ-mlv.fr

$\stackrel{\Perp}{\mapsto} \quad \#$

\# Version REDTEN-060527

\#

\# Needed programs: MAPLE 9.5 or MAPLE 10

\#

\# Input parameters $M$ and NDIM1 must be set in calling program.

\#

\# 1) Load subroutines and libraries:

time $0:=\operatorname{time}()$ :

read 'redtenlib.cmd' :

with (linalg) : 
with (LinearAlgebra):

outputfilename:=cat ("turm-", M, "-", NDIM1, ". out") :

outputfile:=fopen (outputfilename, WRITE):

\#

\# 2) Define tensor space and key:

KDATA : $=\operatorname{defk}(M, N D I M 1)$ :

\# Procedure defk returns 2 items:

NDIMm:=KDATA[1]: \# tensor space dimension

KEY:=eval (KDATA[2]): \# matrix MxNDIMm defining the tensor space.

prtime(outputfile,2, "TURM: key definition",time0):

Nㅗㅇ \# 3) Define permutation matrices of order M, the "turmdarstellung":

SDATA : $=\operatorname{Sm}(M)$ :

\# Procedure Sm returns a list of 5 items:

MORDR:=SDATA[1]: \# the group order .

MIRRP:=SDATA [2]: \# the number of irreductible representations of the group.

SCHAR:=SDATA [3] : \# a MIRRPXMORDR matrix containing the character table.

$\operatorname{SLABE}:=\operatorname{array}(1 \ldots$ MIRRP) :

SLABE:=evalm(SDATA[4]): \# a 1..NIRRP array containing usual labels of the irr. rep.

PMAT:=SDATA[5]: \# a list of matrices of the form PMAT $[n][k, j]$,

\# where $\mathrm{n}$ is the number of the operation,

\# $\quad \mathrm{k}$ and $\mathrm{j}$ are indices from 1..M, the "turmdarstellung". 
prtime (outputfile,3, "TURM: setup turmdarstellung",time0):

\# 4) Get representation of $\mathrm{Sm}$ in $\mathrm{Vm}$ TREP : =evalm (SmInVm(M , NDIMm, MORDR , KEY , PMAT) ) :

\# Procedure SmInVm returns 1 item: a list of M! matrices NDIMmxNDIMm \# defining the permutation matrices of $M$ elements in \# tensor space $\mathrm{V} * * \mathrm{M}$.

\# prtime(outputfile,4,"TURM: setup permutation matrices",time0):

\# 5) Reduce permutation representation in Sm, get only A1 REDTS : =redA1 (MORDR, MIRRP , SCHAR, NDIMm, TREP, ti me0) :

이 \# Procedure redA1 returns a list of 2 items: REDF:=REDTS[1]: \# the list 1 ..MIRRP of reduction factors TS : =matrix (NDIMm, NDIMm) :

TS:=REDTS[2]: \# the NDIMmxREDF[1] transformation matrix into the \# irreductible space A1 prtime(outputfile,5,"TURM: reduction of perm. matrices",time0):

\# 6) Saving data: REDSM : = [M, NDIM1, MIRRP, eval (REDF), evalm(TS)] : datafile:=cat ("turmdata-" , M, "-" , NDIM1, ".res") : save REDSM, datafile: prtime(outputfile,6, "TURM: saving data",time0): 
fclose (outputfilename): 


\section{C redtenlib.cmd}

\# This file is a library of routines to be used within program REDTEN.

\#

\# Authors: R. Marquardt and K. Sagui

\# Université de Marne-la-Vallée

\# 5 Bd Descartes, 77454 Marne-la-Vallée CEDEX 2

\# email for contact: roberto.marquardt@univ-mlv.fr

\#

\# Version REDTEN-060527

लै \#

\# Needed programs: MAPLE 9.5 or MAPLE 10

\#

prtime: $=\operatorname{proc}(f i l e, i$, txt: : string, time $)$

local n, rtime, H,M, S :

rtime: =time()-time0:

$\mathrm{H}:=\operatorname{trunc}($ rtime $/ 3600$.) :

$M:=\operatorname{trunc}(($ rtime $-H * 3600$. $) / 60$. ) :

$\mathrm{S}:=$ rtime- $(\mathrm{H} * 3600 .+\mathrm{M} * 60$.$) :$

fprintf(file, "Step \%-5.0f ( $\%-34 \mathrm{~s})$ done; \%3.0f h \%2.0f m \%2.3f s $\backslash \mathrm{n}$ ",i,txt,H,M,S);

printf("Step \%-5.0f ( $\%-34 \mathrm{~s})$ done; \%3.0f h \%2.0f m \%2.3f $\mathrm{s} \backslash \mathrm{n}$ ", i,txt,H,M,S); 
end proc:

irrep: $=\operatorname{proc}(G)$

local i, n, ng, nirrep, chartab, label, Girr, nnb, RESULT , a , b , c , d, e, f, g, h:

"Definition of irreductible representations.":

"Currently available: CS, C2v, C3v, Td":

$\mathrm{a}:=1 / 2$

$\mathrm{b}:=\operatorname{sqrt}(3) / 2$ :

$c:=1 / 3$ :

$\mathrm{d}:=\operatorname{sqrt}(2) / 3$ :

e: $=\operatorname{sqrt}(6) / 3$ :

$f:=5 / 6$ :

$\mathrm{g}:=\operatorname{sqrt}(3) / 6$ :

$\mathrm{h}:=\operatorname{sqrt}(8) / 3$ :

if $\left(\mathrm{G}={ }^{\prime} \mathrm{CS}{ }^{\prime}\right)$ then

$\mathrm{nnb}:=1$ : \# number of operation with non-diagonal representation

$\mathrm{ng}:=2: \quad$ \# group order

nirrep:=2: number of irreps (number of classes)

chartab: =matrix (nirrep, $\mathrm{ng},[[1,1]$,

$$
[1,-1]]):
$$

label :=array (1 . nirrep) :

\#

\#1st irrep 


\section{Page 59 of 107}

\section{Molecular Physics}

label $[1]:=A$ :

$\operatorname{Girr}[1][1]:=\operatorname{matrix}(1,1,[[1]])$ :

Girr [1] [2] :=matrix $(1,1,[[1]])$ :

\#

\#2nd irrep

label [2] :=B :

$\operatorname{Girr}[2][1]:=\operatorname{matrix}(1,1,[[1]])$ :

$\operatorname{Girr}[2][2]:=\operatorname{matrix}(1,1,[[-1]])$ :

elif $\left(\left(G={ }^{\prime} \mathrm{C} 2 \mathrm{v}^{\prime}\right)\right.$ or $\left.\left(\mathrm{G}={ }^{\prime} \mathrm{S} 2{ }^{\prime}\right)\right)$ then

nnb:=1: \# number of operation with non-diagonal representation

$\mathrm{ng}:=4: \quad$ \# group order

$\stackrel{N}{ }$ nirrep:=4:\# number of irreps (number of classes)

chartab:=matrix (nirrep, $n g,[[1,1,1,1]$,

$$
\begin{aligned}
& {[1,1,-1,-1],} \\
& {[1,-1,-1,1],} \\
& [1,-1,1,-1]]):
\end{aligned}
$$

label:=array $(1 \ldots$ nirrep $)$ :

\#

\#1st irrep

label [1] :=A1:

$\operatorname{Girr}[1][1]:=\operatorname{matrix}(1,1,[[1]])$ :

$\operatorname{Girr}[1][2]:=\operatorname{matrix}(1,1,[[1]])$ :

$\operatorname{Girr}[1][3]:=\operatorname{matrix}(1,1,[[1]])$ : 
$\operatorname{Girr}[1][4]:=\operatorname{matrix}(1,1,[[1]]):$

\#

\# \#2nd irrep

label [2]:=A2:

$\operatorname{Girr}[2][1]:=\operatorname{matrix}(1,1,[[1]])$ :

$\operatorname{Girr}[2][2]:=\operatorname{matrix}(1,1,[[1]])$ :

$\operatorname{Girr}[2][3]:=\operatorname{matrix}(1,1,[[-1]])$ :

$\operatorname{Girr}[2][4]:=\operatorname{matrix}(1,1,[[-1]])$ :

\#3rd irrep

label [3] : =B1 :

$\operatorname{Girr}[3][1]:=\operatorname{matrix}(1,1,[[1]])$ :

Girr [3] [2] :=matrix $(1,1,[[-1]])$ :

$\operatorname{Girr}[3][3]:=\operatorname{matrix}(1,1,[[-1]])$ :

$\operatorname{Girr}[3][4]:=\operatorname{matrix}(1,1,[[1]])$ :

\#4th irrep

label [4] : =B2 :

$\operatorname{Girr}[4][1]:=\operatorname{matrix}(1,1,[[1]])$ :

$\operatorname{Girr}[4][2]:=\operatorname{matrix}(1,1,[[-1]])$ :

$\operatorname{Girr}[4][3]:=\operatorname{matrix}(1,1,[[1]])$ :

$\operatorname{Girr}[4][4]:=\operatorname{matrix}(1,1,[[-1]])$ :

elif $\left(\left(G={ }^{\prime} C 3 v^{\prime}\right)\right.$ or $\left.\left(G={ }^{\prime} S 3^{\prime}\right)\right)$ then

$\mathrm{nnb}:=2$ : \# number of operation with non-diagonal representation 
$\mathrm{ng}:=6: \quad$ \# group order

nirrep:=3: number of irreps (number of classes)

chartab:=matrix (nirrep, ng, $[[1,1,1,1,1,1]$,

$[1,1,1,-1,-1,-1]$,

$[2,-1,-1,0,0,0]])$ :

\#

label:=array (1...nirrep) :

\#1st irrep

label [1] :=A1:

$\operatorname{Girr}[1][1]:=\operatorname{matrix}(1,1,[[1]])$ :

$\operatorname{Girr}[1][2]:=\operatorname{matrix}(1,1,[[1]])$ :

$\operatorname{Girr}[1][3]:=\operatorname{matrix}(1,1,[[1]])$ :

$\operatorname{Girr}[1][4]:=\operatorname{matrix}(1,1,[[1]])$ :

$\operatorname{Girr}[1][5]:=\operatorname{matrix}(1,1,[[1]])$ :

$\operatorname{Girr}[1][6]:=\operatorname{matrix}(1,1,[[1]])$ :

\#

\#2nd irrep

label [2] :=A2 :

Girr [2] [1] :=matrix $(1,1,[[1]])$ :

Girr [2] [2] :=matrix $(1,1,[[1]])$ :

$\operatorname{Girr}[2][3]:=\operatorname{matrix}(1,1,[[1]])$ :

$\operatorname{Girr}[2][4]:=\operatorname{matrix}(1,1,[[-1]])$ :

$\operatorname{Girr}[2][5]:=\operatorname{matrix}(1,1,[[-1]])$ : 
$\operatorname{Girr}[2][6]:=\operatorname{matrix}(1,1,[[-1]])$ :

\# \#3rd irrep

label [3] : =E :

$\operatorname{Girr}[3][1]:=\operatorname{matrix}(2,2,[[\quad 1, \quad 0]$,

[ $0, \quad 1]])$ :

Girr [3] [2] :=matrix $(2,2,[[\quad-1 / 2,-\operatorname{sqrt}(3) / 2]$,

$$
[\operatorname{sqrt}(3) / 2, \quad-1 / 2]]) \text { : }
$$

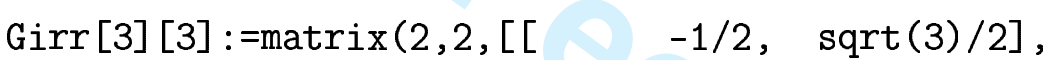

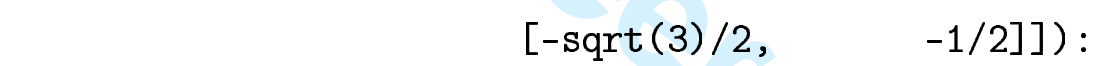




$$
[1,-1,-1,-1,-1,-1,-1,1,1,1,1,1 \text {, }
$$

$1,1,1,-1,-1,-1,-1,-1,-1,1,1,1]$,

$[2,0,0,0,0,0,0,-1,-1,-1,-1,-1$,

$-1,-1,-1,0,0,0,0,0,0,2,2,2]$,

$[3,-1,-1,-1,-1,-1,-1,0,0,0,0,0$,

$0,0,0,1,1,1,1,1,1,-1,-1,-1]$,

$[3,1,1,1,1,1,1,0,0,0,0,0$,

$0,0,0,-1,-1,-1,-1,-1,-1,-1,-1,-1]])$ :

label: =array (1..nirrep) :

\#

\#1st irrep

label [1] :=A1:

for $\mathrm{n}$ from 1 to $\mathrm{ng}$ do

$\operatorname{Girr}[1][n]:=\operatorname{matrix}(1,1,[[1]])$ :

od:

\#2nd irrep

label [2] :=A2:

Girr [2] [1] :=matrix $(1,1,[[1]])$ :

Girr[2] [2] :=matrix $(1,1,[[-1]])$ :

Girr [2] [3] :=matrix $(1,1,[[-1]])$ :

Girr[2] [4] :=matrix $(1,1,[[-1]])$ :

Girr [2] [5] :=matrix $(1,1,[[-1]])$ : 


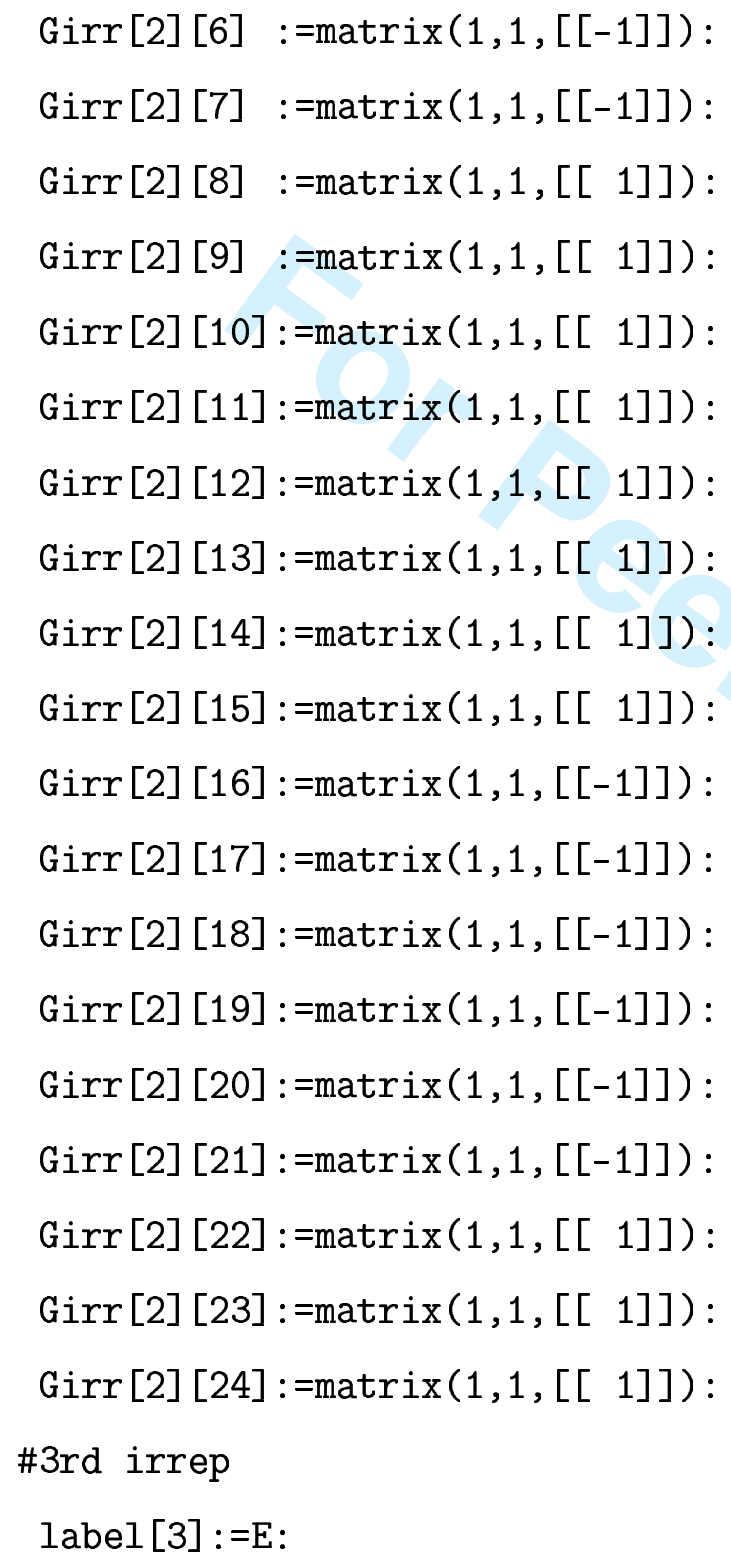




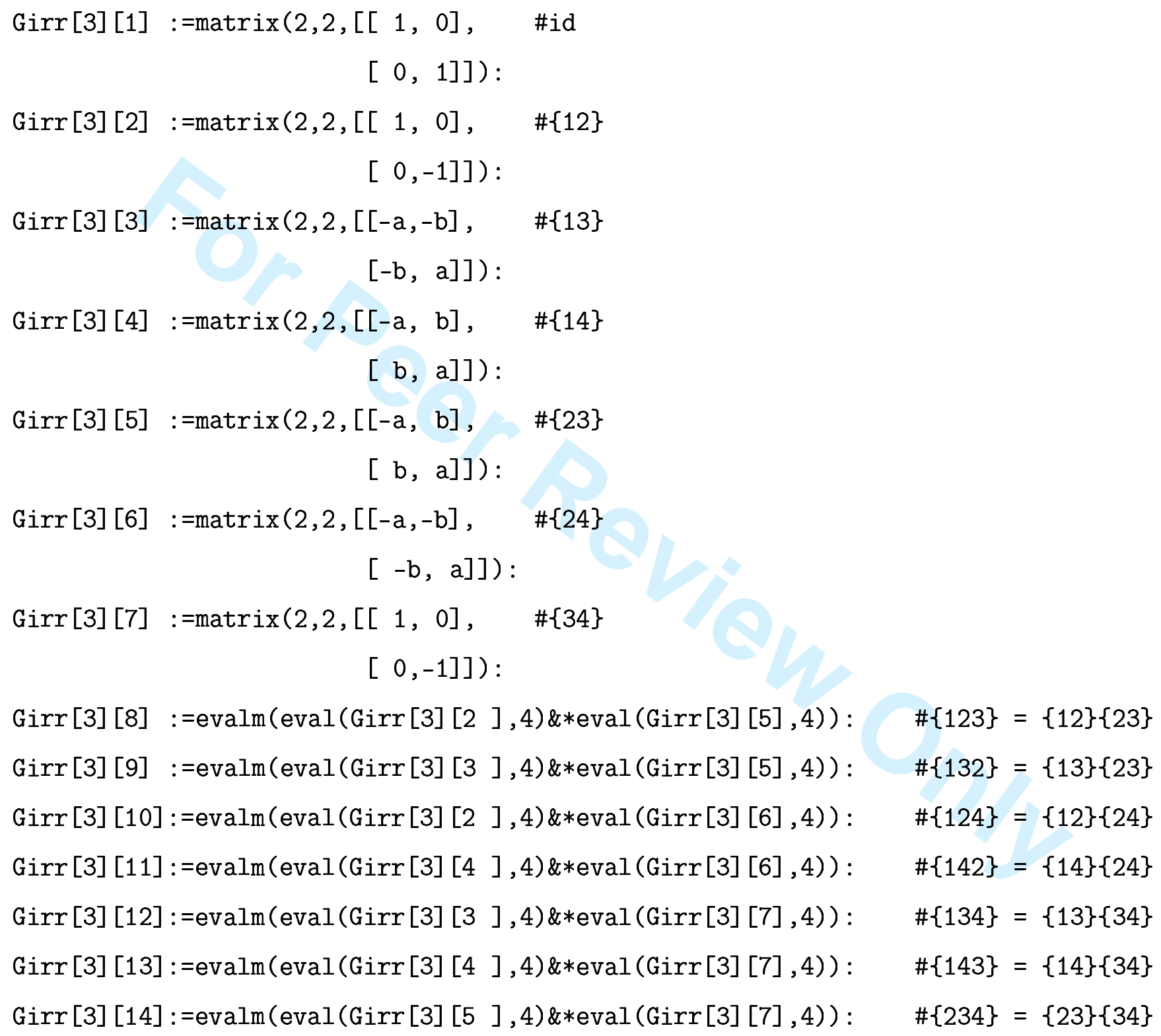




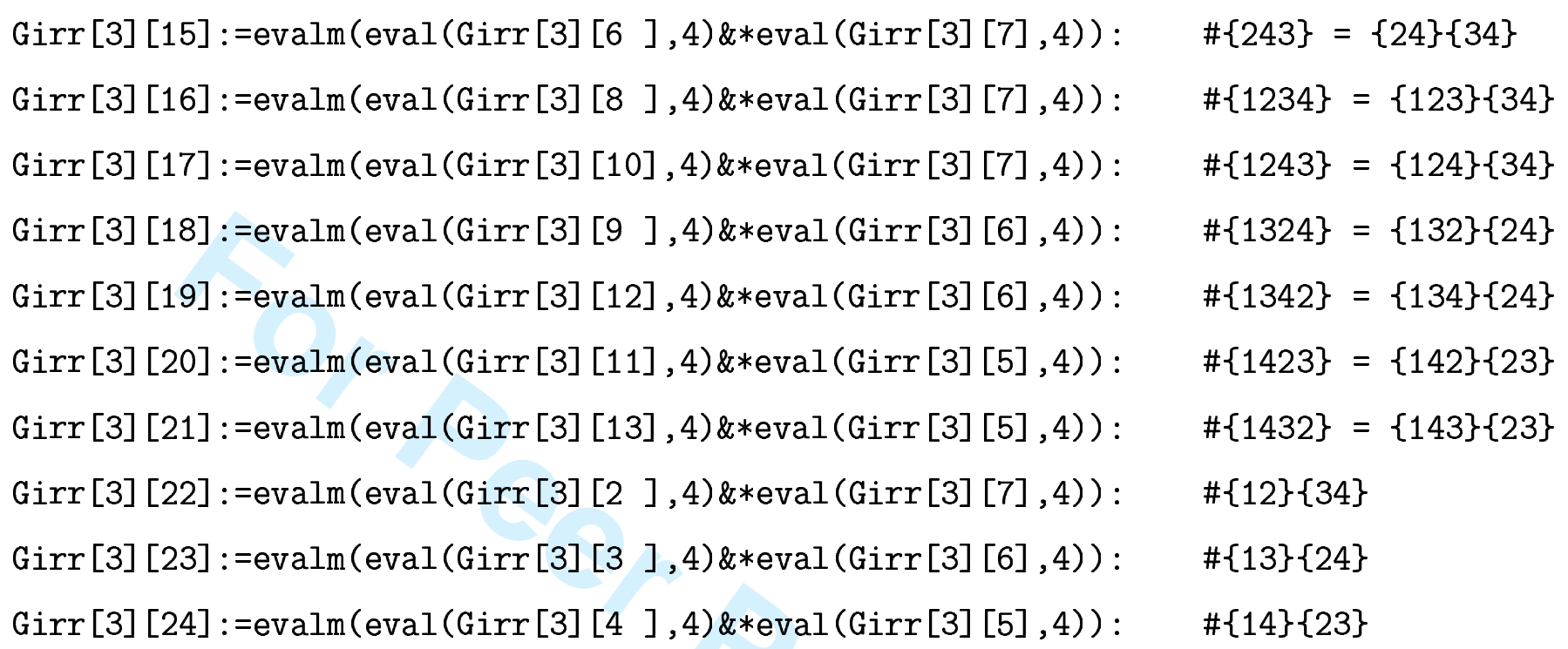




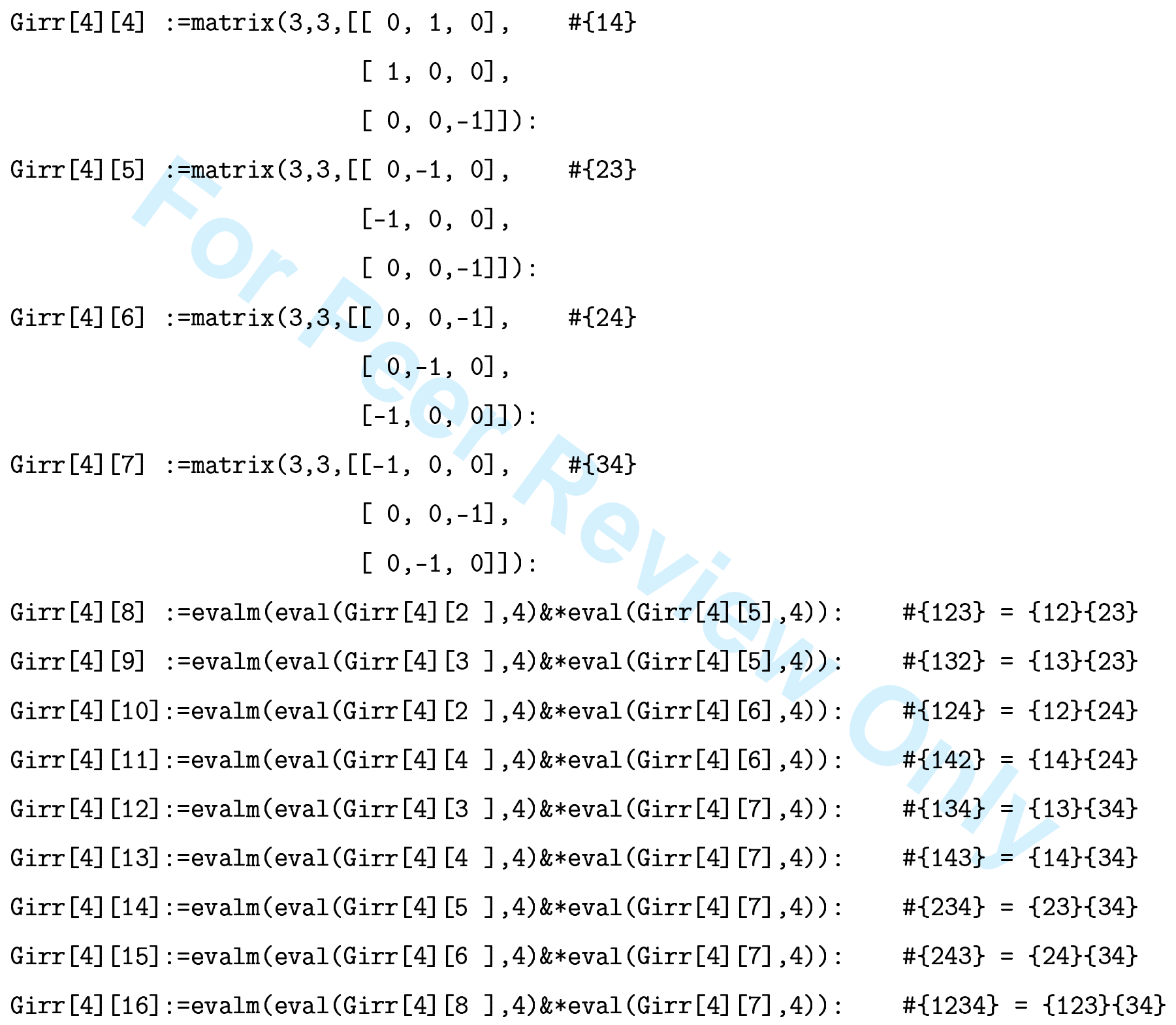




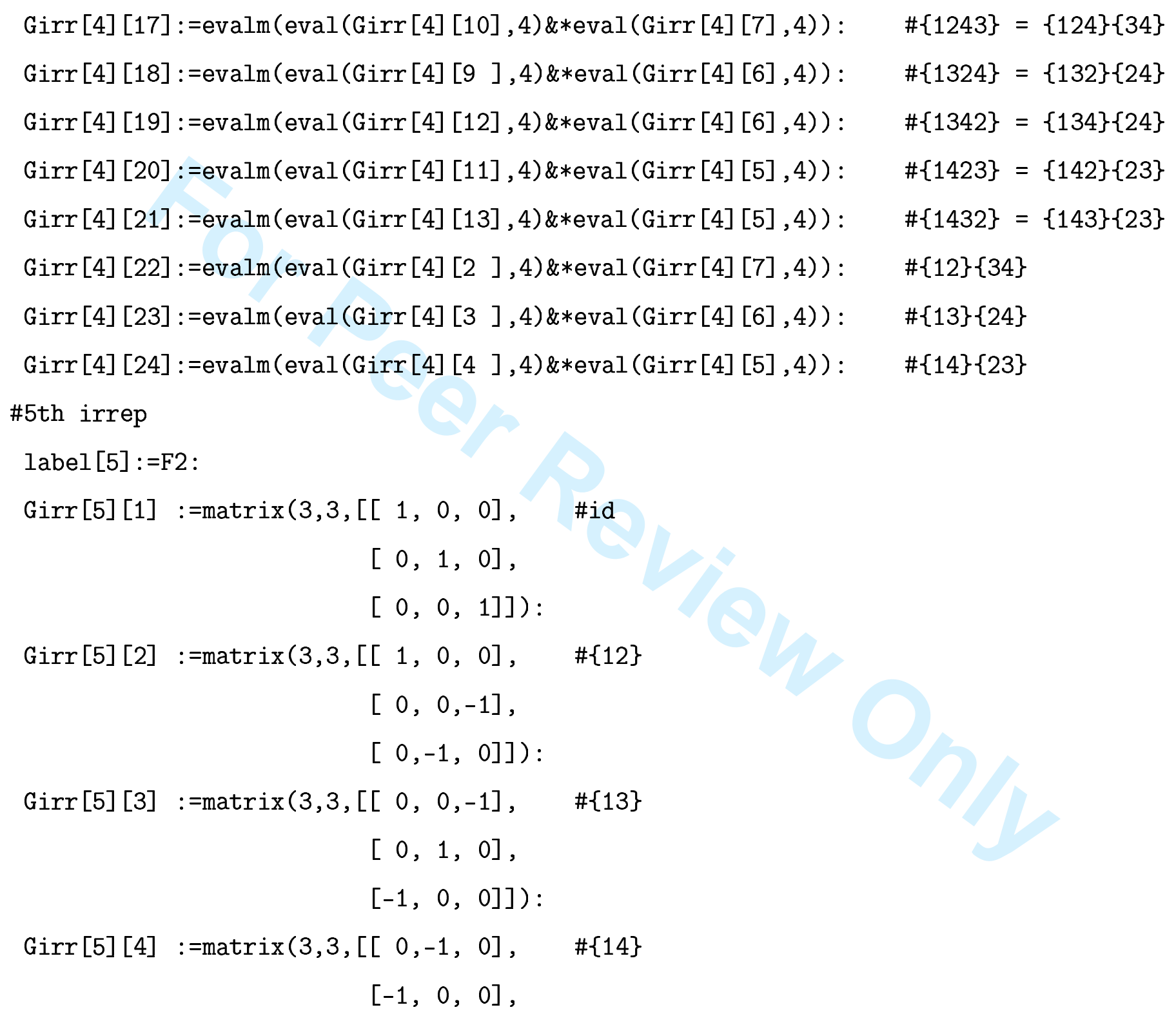




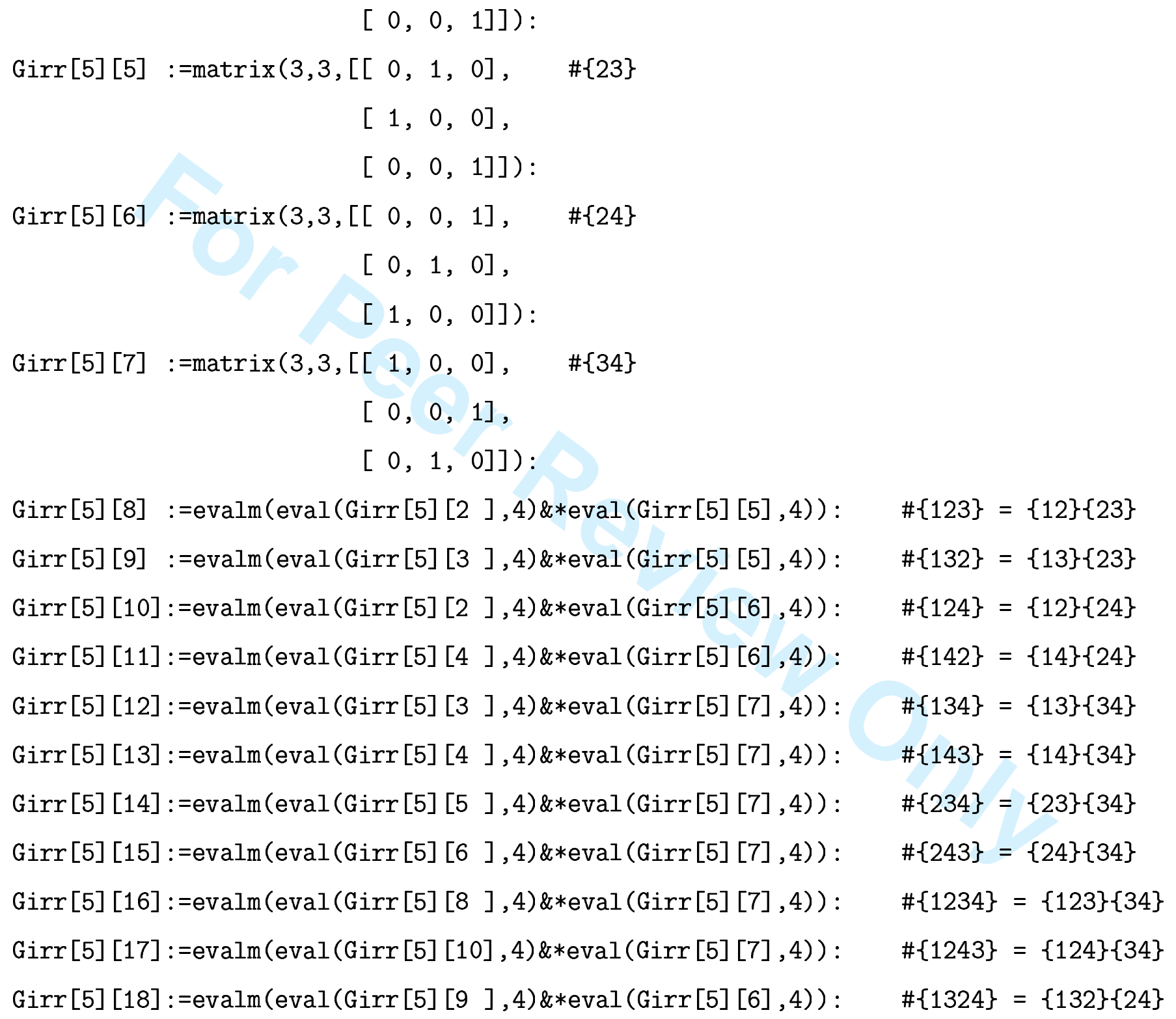




$$
\begin{array}{ll}
\operatorname{Girr}[5][19]:=\operatorname{evalm}(\operatorname{eval}(\operatorname{Girr}[5][12], 4) \& * \operatorname{eval}(\operatorname{Girr}[5][6], 4)): & \#\{1342\}=\{134\}\{24\} \\
\operatorname{Girr}[5][20]:=\operatorname{evalm}(\operatorname{eval}(\operatorname{Girr}[5][11], 4) \& * \operatorname{eval}(\operatorname{Girr}[5][5], 4)): & \#\{1423\}=\{142\}\{23\} \\
\operatorname{Girr}[5][21]:=\operatorname{evalm}(\operatorname{eval}(\operatorname{Girr}[5][13], 4) \& * \operatorname{eval}(\operatorname{Girr}[5][5], 4)): & \#\{1432\}=\{143\}\{23\} \\
\operatorname{Girr}[5][22]:=\operatorname{evalm}(\operatorname{eval}(\operatorname{Girr}[5][2], 4) \& * \operatorname{eval}(\operatorname{Girr}[5][7], 4)): & \#\{12\}\{34\} \\
\operatorname{Girr}[5][23]:=\operatorname{evalm}(\operatorname{eval}(\operatorname{Girr}[5][3], 4) \& * \operatorname{eval}(\operatorname{Girr}[5][6], 4)): & \#\{13\}\{24\} \\
\operatorname{Girr}[5][24]:=\operatorname{evalm}(\operatorname{eval}(\operatorname{Girr}[5][4], 4) \& * \operatorname{eval}(\operatorname{Girr}[5][5], 4)): & \#\{14\}\{23\}
\end{array}
$$

for $i$ from 3 to 5 do

for $\mathrm{n}$ from 1 to $\mathrm{ng}$ do

$\operatorname{Girr}[\mathrm{i}][\mathrm{n}]:=\operatorname{map}($ combine, Girr [i] [n], radical, symbolic) :

od:

$\infty_{\infty}^{\infty}$

od:

else

print("Group ",G," not identified; program stopped.");

stop

end if :

[ng, nirrep, chartab, label, Girr, nnb] :

end proc:

defk : $=\operatorname{proc}(m, n d 1)$

local ndm, nd, mm, $k, j, j 1, j 2, j 3, j 4, k e y$ :

"Definition of order m tensor space key.":

$\mathrm{ndm}:=\mathrm{nd} 1 * * \mathrm{~m}$ : 


\section{Page 71 of 107}

\section{Molecular Physics}

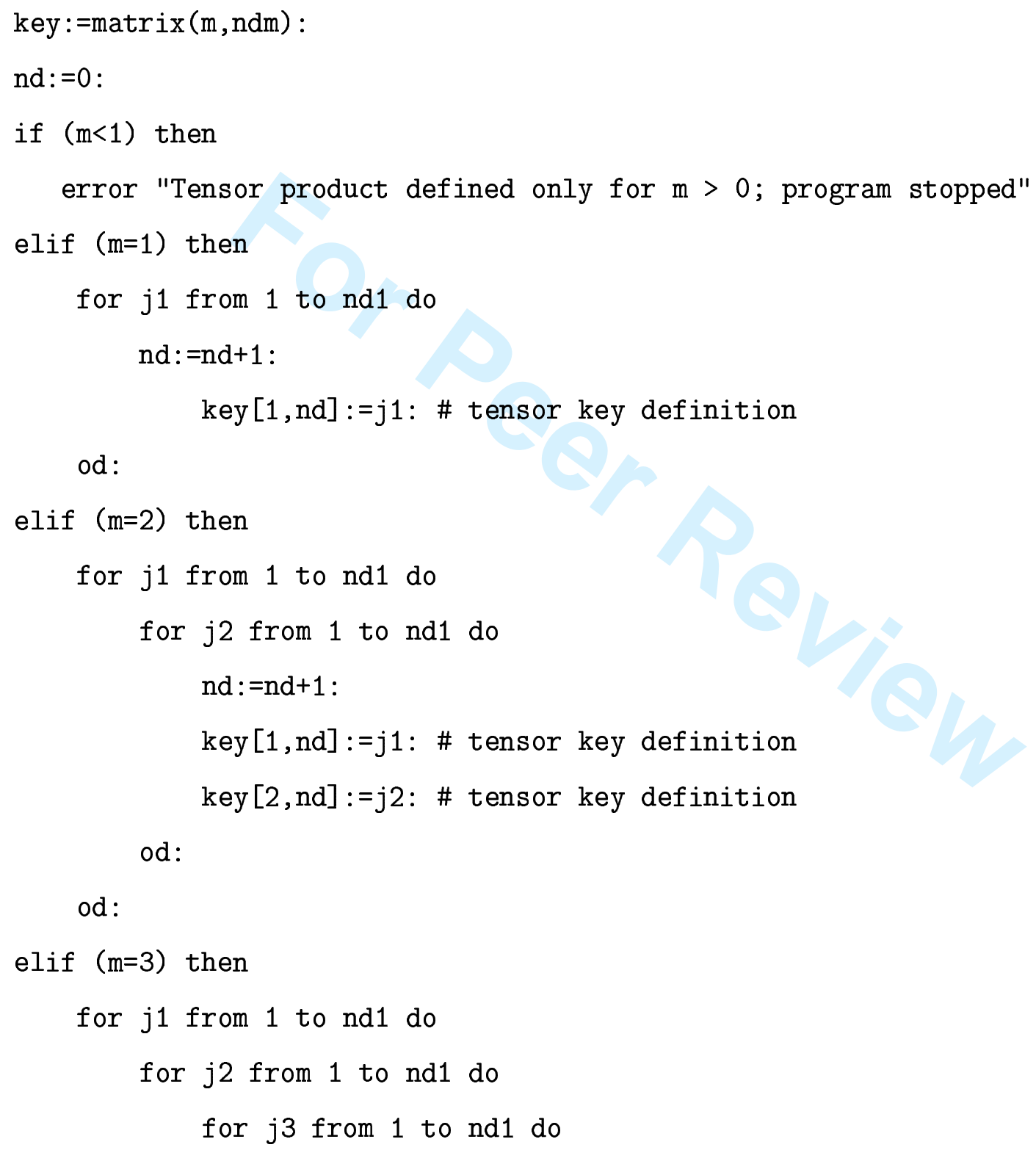


nd : $=\mathrm{nd}+1$ :

key $[1, \mathrm{nd}]:=\mathrm{j} 1$ : \# tensor key definition

key $[2, \mathrm{nd}]:=\mathrm{j} 2$ : \# tensor key definition

$\operatorname{key}[3, n d]:=j 3$ : \# tensor key definition

od:

od:

od:

elif $(m=4)$ then

for $j 1$ from 1 to nd1 do

for $j 2$ from 1 to nd1 do

for $j 3$ from 1 to nd1 do

for $j 4$ from 1 to nd1 do

nd: $=$ nd +1 :

key[1,nd]:=j1: \# tensor key definition

key[2,nd]:=j2: \# tensor key definition

key[3,nd]:=j3: \# tensor key definition

key $[4, n d]:=j 4$ : \# tensor key definition

od:

od:

od:

od : 


\section{Page 73 of 107}

\section{Molecular Physics}

else

error "Tensor product definition limited to $\mathrm{m}=4$; program stopped"

end if:

[ndm, key] :

end proc:

repm : $=\operatorname{proc}(m, n d m$, key, $\mathrm{G1})$

local mm,k, j, Gm:

"Definition of group representations in order $m$ tensor space.":

$\mathrm{Gm}:=\operatorname{matrix}(\mathrm{ndm}, \mathrm{ndm})$ :

for $\mathrm{k}$ from 1 to $\mathrm{ndm}$ do

for $j$ from 1 to $\mathrm{ndm}$ do

$\operatorname{Gm}[k, j]:=1$ :

for $\mathrm{mm}$ from 1 to $\mathrm{m}$ do

$\operatorname{Gm}[\mathrm{k}, \mathrm{j}]:=\mathrm{Gm}[\mathrm{k}, \mathrm{j}] * \mathrm{G} 1[\mathrm{key}[\mathrm{mm}, \mathrm{k}], \operatorname{key}[\mathrm{mm}, j]]$ :

$\operatorname{Gm}[k, j]:=\operatorname{simplify}(\mathrm{Gm}[k, j])$ :

od :

od :

od:

\# Gm:=map (combine, $\mathrm{Gm}$, radical, symbolic):

\# combine needs 3 times more memory!

$\mathrm{Gm}$ : 
end proc:

simtra $:=\operatorname{proc}(\mathrm{T}, \mathrm{TT}, \mathrm{G})$

local Gs:

description "similarity transformation":

Gs : $=$ evalm $(\& *(T T, G, T))$ :

\# Gs:=linalg[multiply] (TT, G, T) :

Gs : $=\operatorname{map}($ simplify, Gs) :

\# Gs : $=\operatorname{map}($ combine, Gs, radical, symbolic) :

end proc:

$\mathrm{Sm}:=\operatorname{proc}(\mathrm{m})$

local MP, chartab,ng, nirrep, label:

description " Turmdarstellung of symmetric group Sm":

if $(m<1)$ then

print("Permutations defined only for $\mathrm{m}>0$; program stopped");

stop

elif ( $m>4$ ) then \#work in progress below for $m=5$

print("Permutation matrices limited to $\mathrm{m}=4$; program stopped");

stop

end if :

$\mathrm{ng}:=$ factorial $(\mathrm{m})$ :

if $(m=1)$ then 


\section{Page 75 of 107}

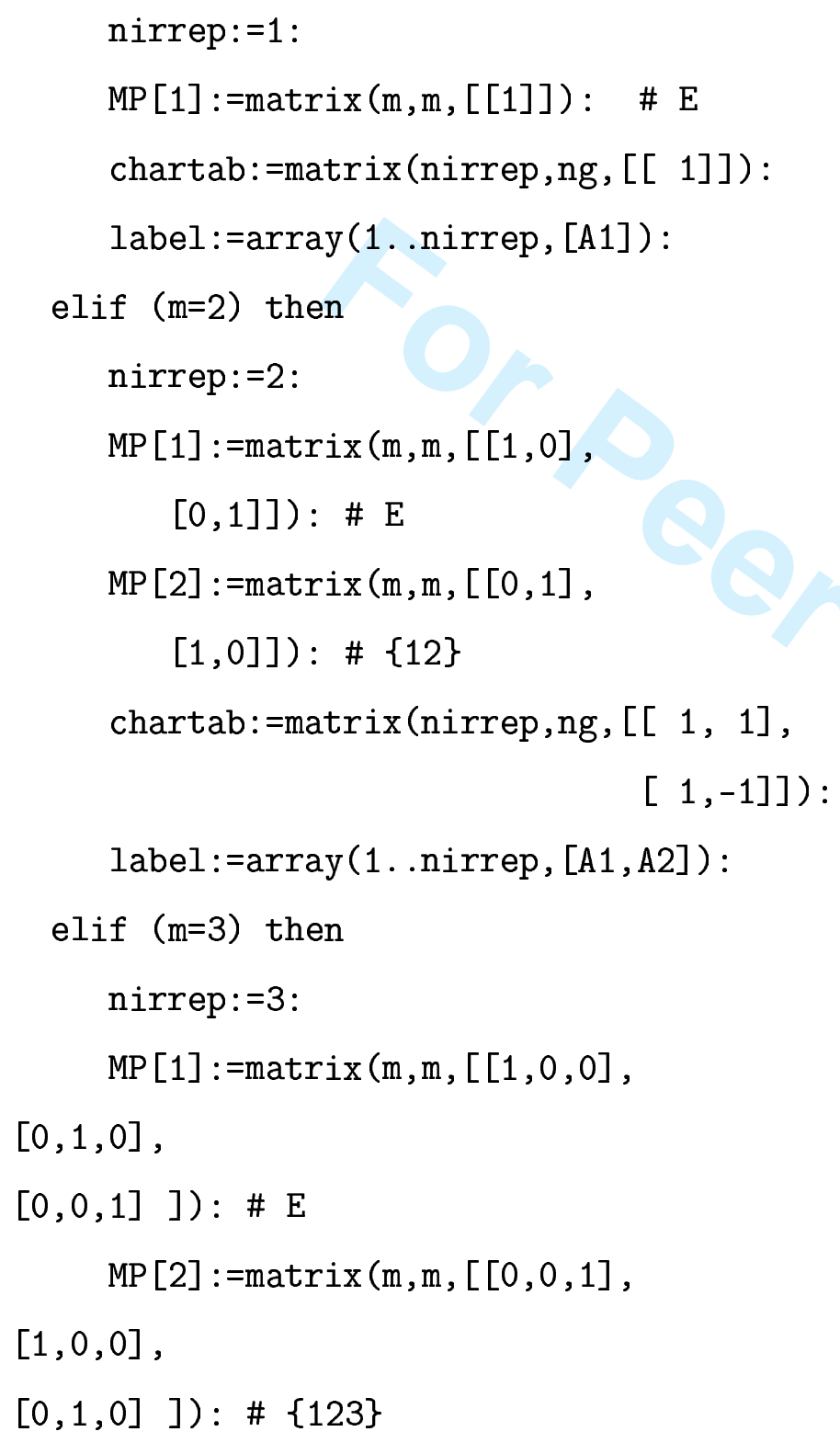




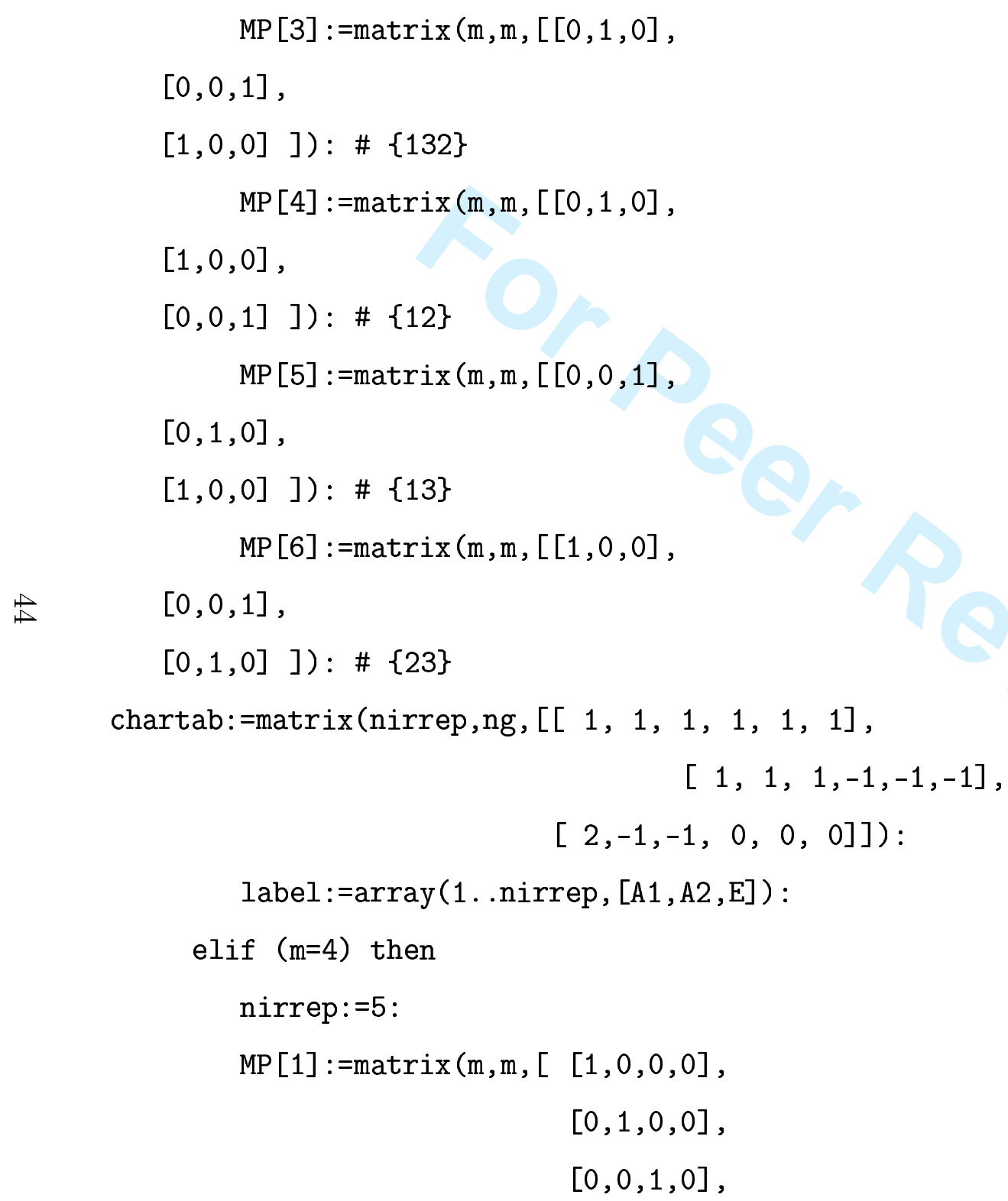




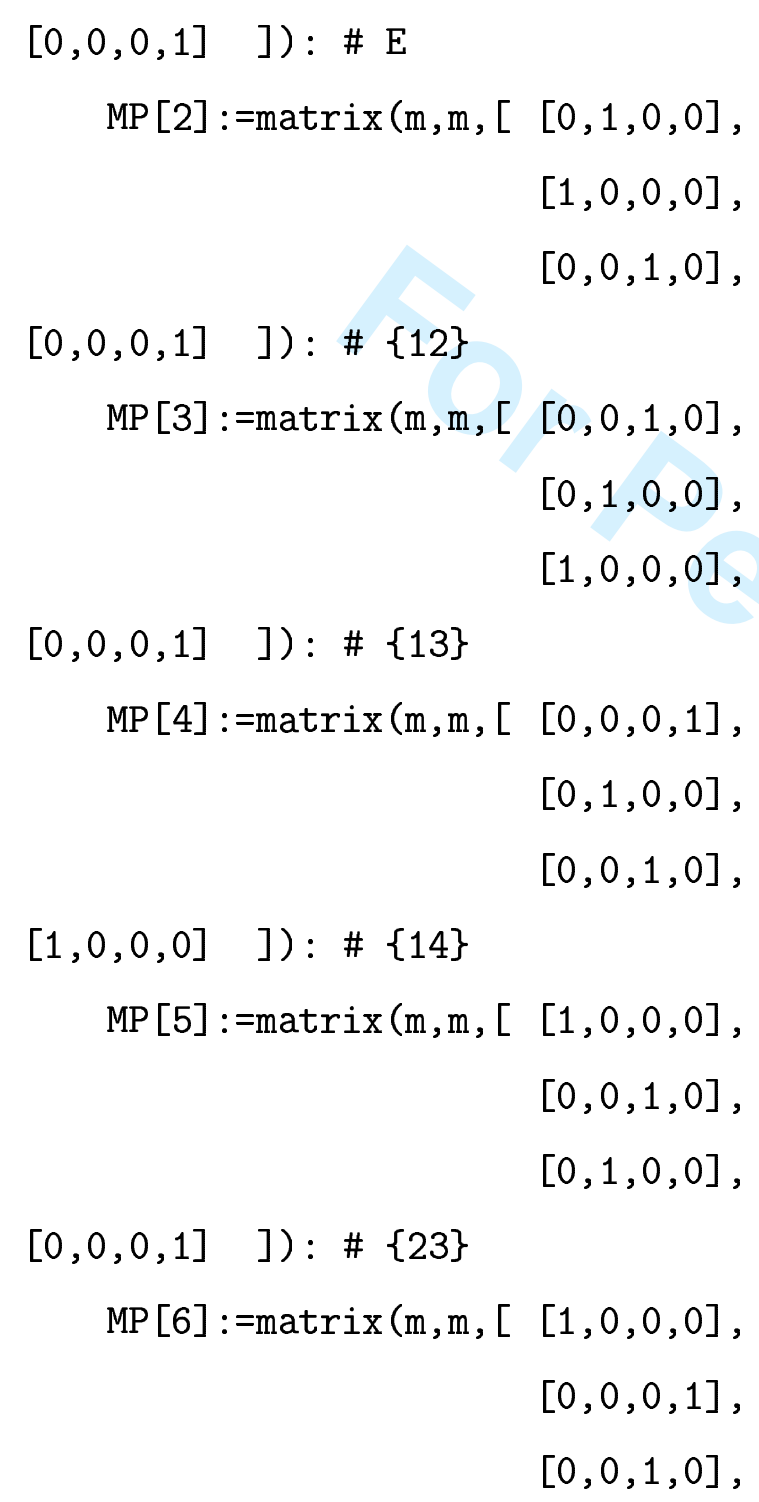


MP $[7]:=\operatorname{matrix}(\mathrm{m}, \mathrm{m},[[1,0,0,0]$,

$[0,1,0,0]$,

$[0,0,0,1]$,

$[0,0,1,0] \quad]): \#\{34\}$

MP [8] : =matrix $(m, m,[[0,0,1,0]$,

$[1,0,0,0]$,

$[0,1,0,0]$,

$[0,0,0,1]]): \#\{123\}$

$\operatorname{MP}[9]:=\operatorname{matrix}(\mathrm{m}, \mathrm{m},[[0,1,0,0]$,

$[0,0,1,0]$,

$[1,0,0,0]$,

$[0,0,0,1]]): \#\{132\}$

$\operatorname{MP}[10]:=\operatorname{matrix}(\mathrm{m}, \mathrm{m},[[0,0,0,1]$,

$[1,0,0,0]$,

$[0,0,1,0]$,

$[0,1,0,0] \quad]): \#\{124\}$

MP $[11]:=\operatorname{matrix}(m, m,[[0,1,0,0]$,

$[0,0,0,1]$,

$[0,0,1,0]$,

$[1,0,0,0] \quad]): \#\{142\}$

$\operatorname{MP}[12]:=\operatorname{matrix}(\mathrm{m}, \mathrm{m},[[0,0,0,1]$, 


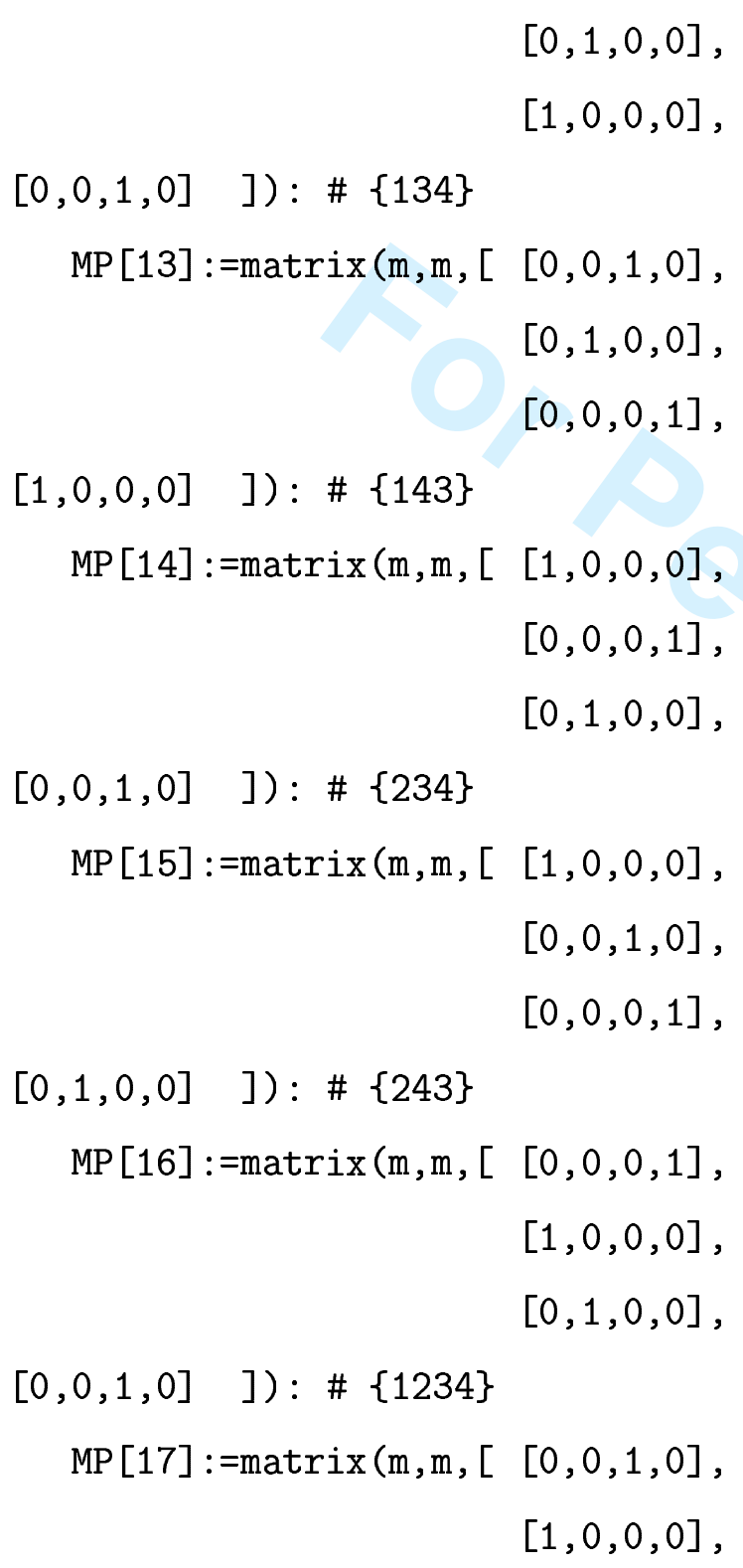




$$
[0,0,0,1]
$$

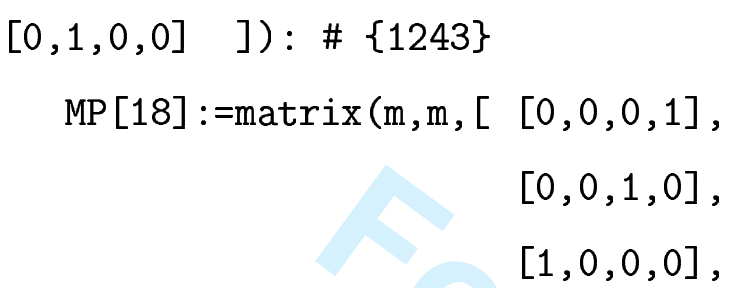




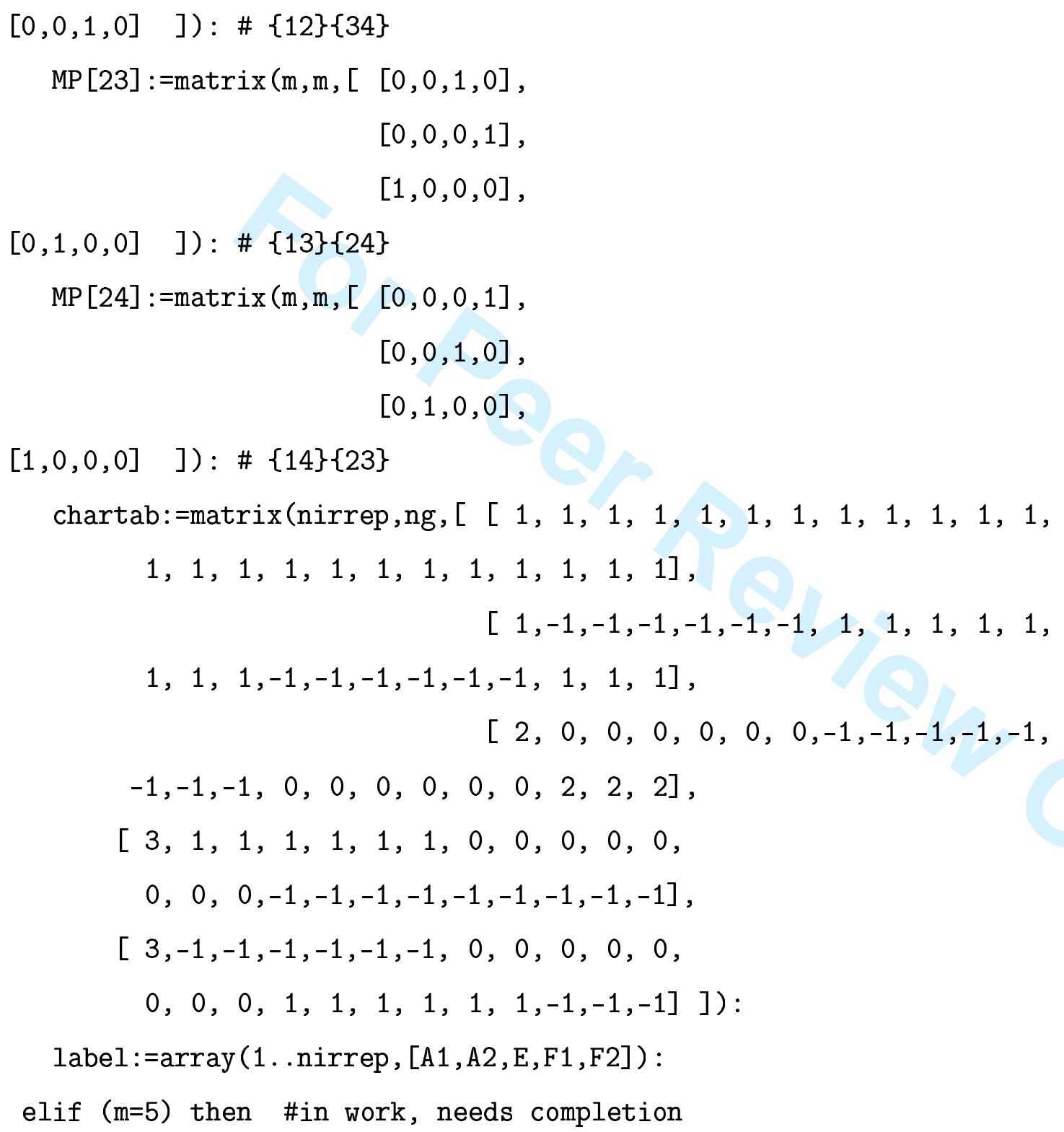




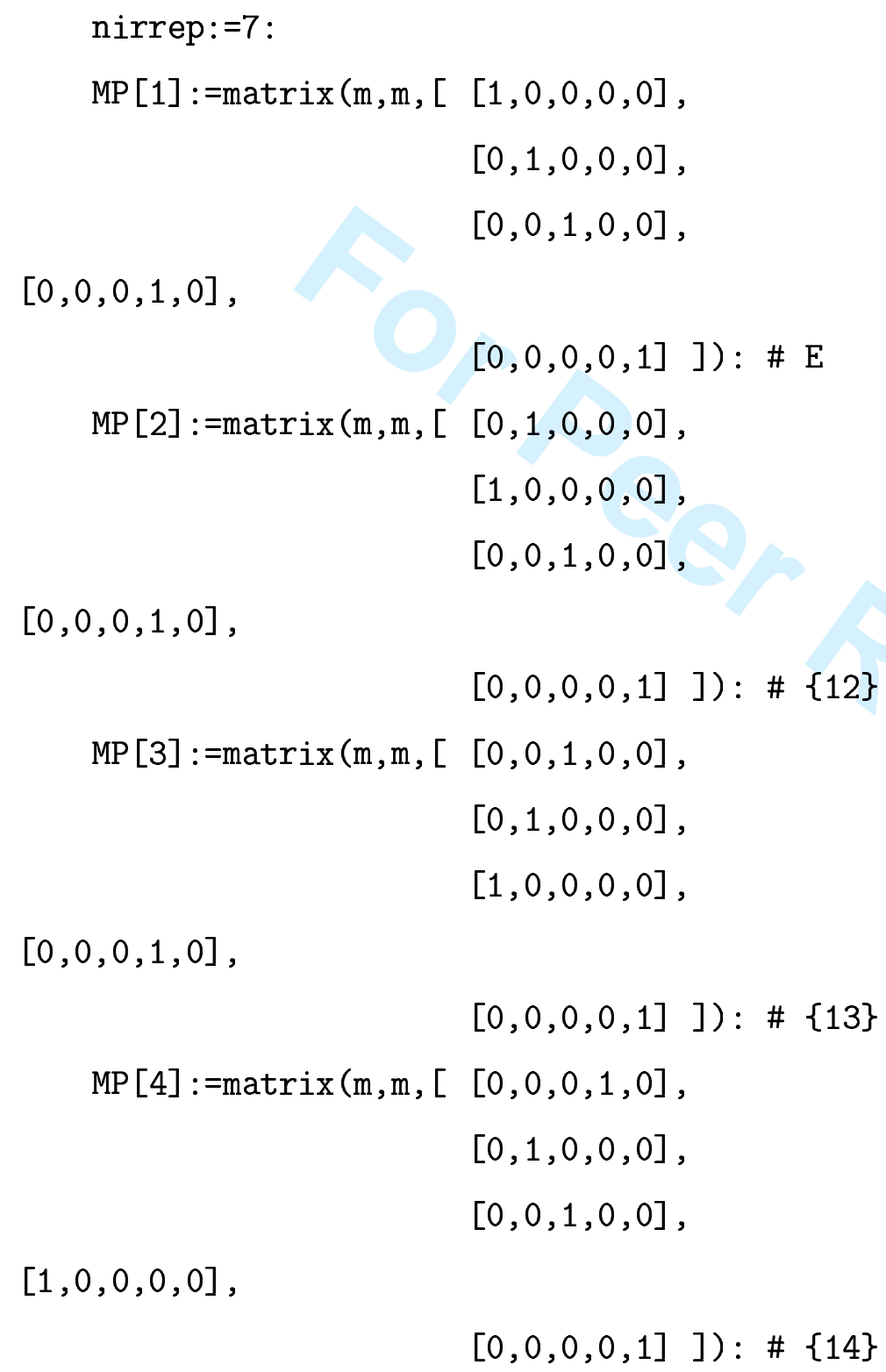


MP [5] : =matrix $(\mathrm{m}, \mathrm{m},[[0,0,0,0,1]$,

$[0,1,0,0,0]$,

$[0,0,1,0,0]$,

$[0,0,0,1,0]$,

$[1,0,0,0,0]]): \#\{15\}$

$\operatorname{MP}[6]:=\operatorname{matrix}(\mathrm{m}, \mathrm{m},[[1,0,0,0,0]$,

$[0,0,1,0,0]$,

$[0,1,0,0,0]$,

$[0,0,0,1,0]$,

$[0,0,0,0,1]]): \#\{23\}$

$\stackrel{\vartheta}{\bullet}$

MP $[7]:=\operatorname{matrix}(m, m,[[1,0,0,0,0]$,

$[0,0,0,1,0]$,

$[0,0,1,0,0]$,

$[0,1,0,0,0]$,

$[0,0,0,0,1]]): \#\{24\}$

MP [8] :=matrix (m, m, $[[1,0,0,0,0]$,

$[0,0,0,0,1]$,

$[0,0,1,0,0]$,

$[0,0,0,1,0]$,

$[0,1,0,0,0]]): \#\{25\}$

MP $[9]:=\operatorname{matrix}(m, m,[[1,0,0,0,0]$, 


$$
\begin{aligned}
& {[0,1,0,0,0],} \\
& {[0,0,0,1,0],}
\end{aligned}
$$

$[0,0,1,0,0]$

$$
[0,0,0,0,1]]): \#\{34\}
$$

MP $[10]:=\operatorname{matrix}(\mathrm{m}, \mathrm{m},[[1,0,0,0,0]$,

$$
\begin{aligned}
& {[0,1,0,0,0] \text {, }} \\
& {[0,0,0,0,1],}
\end{aligned}
$$

$[0,0,0,1,0]$,

$$
[0,0,1,0,0]]): \#\{35\}
$$

$\operatorname{MP}[11]:=\operatorname{matrix}(\mathrm{m}, \mathrm{m},[[1,0,0,0,0]$,

$$
[0,1,0,0,0] \text {, }
$$$$
[0,0,1,0,0] \text {, }
$$

$[0,0,0,0,1]$

$$
[0,0,0,1,0]]): \#\{45\}
$$

MP [12] : =evalm $(\operatorname{eval}(\operatorname{MP}[2], 4) \& * \operatorname{eval}(\operatorname{MP}[9], 4)): \quad \#\{12\}\{34\}$

\#\#\# needs to be completed, 120 operations to be defined as products

\# of the 10 foregoing primitive permutations

chartab : =matrix (nirrep, ng,

$[[1$,

$1,1,1,1,1,1,1,1,1,1$

$1,1,1,1,1,1,1,1,1,1,1,1,1,1,1$, 
$1,1,1,1,1,1,1,1,1,1,1,1,1,1,1,1,1,1,1,1$,

$1,1,1,1,1,1,1,1,1,1,1,1,1,1,1,1,1,1,1,1$,

$1,1,1,1,1,1,1,1,1,1,1,1,1,1,1,1,1,1,1,1,1,1,1,1,1,1,1,1,1,1$,

$1,1,1,1,1,1,1,1,1,1,1,1,1,1,1,1,1,1,1,1,1,1,1,1]$,

$[1$,

$-1,-1,-1,-1,-1,-1,-1,-1,-1,-1$,

$1,1,1,1,1,1,1,1,1,1,1,1,1,1,1$,

$1,1,1,1,1,1,1,1,1,1,1,1,1,1,1,1,1,1,1,1$,

$-1,-1,-1,-1,-1,-1,-1,-1,-1,-1,-1,-1,-1,-1,-1,-1,-1,-1,-1,-1$,

$-1,-1,-1,-1,-1,-1,-1,-1,-1,-1,-1,-1,-1,-1,-1,-1,-1,-1,-1,-1,-1,-1,-1,-1,-1,-1,-1,-1,-1,-1$,

$1,1,1,1,1,1,1,1,1,1,1,1,1,1,1,1,1,1,1,1,1,1,1,1]$,

[4,

$2,2,2,2,2,2,2,2,2,2$,

$0,0,0,0,0,0,0,0,0,0,0,0,0,0,0$,

$1,1,1,1,1,1,1,1,1,1,1,1,1,1,1,1,1,1,1,1$,

$-1,-1,-1,-1,-1,-1,-1,-1,-1,-1,-1,-1,-1,-1,-1,-1,-1,-1,-1,-1$,

$0,0,0,0,0,0,0,0,0,0,0,0,0,0,0,0,0,0,0,0,0,0,0,0,0,0,0,0,0,0$,

$-1,-1,-1,-1,-1,-1,-1,-1,-1,-1,-1,-1,-1,-1,-1,-1,-1,-1,-1,-1,-1,-1,-1,-1]$,

[ 4,

$-2,-2,-2,-2,-2,-2,-2,-2,-2,-2$,

$0,0,0,0,0,0,0,0,0,0,0,0,0,0,0$ 
$1,1,1,1,1,1,1,1,1,1,1,1,1,1,1,1,1,1,1,1$,

$1,1,1,1,1,1,1,1,1,1,1,1,1,1,1,1,1,1,1,1$,

$0,0,0,0,0,0,0,0,0,0,0,0,0,0,0,0,0,0,0,0,0,0,0,0,0,0,0,0,0,0$,

$-1,-1,-1,-1,-1,-1,-1,-1,-1,-1,-1,-1,-1,-1,-1,-1,-1,-1,-1,-1,-1,-1,-1,-1]$,

$[5$,

$1,1,1,1,1,1,1,1,1,1$,

$1,1,1,1,1,1,1,1,1,1,1,1,1,1,1$,

$-1,-1,-1,-1,-1,-1,-1,-1,-1,-1,-1,-1,-1,-1,-1,-1,-1,-1,-1,-1$,

$1,1,1,1,1,1,1,1,1,1,1,1,1,1,1,1,1,1,1,1$,

$-1,-1,-1,-1,-1,-1,-1,-1,-1,-1,-1,-1,-1,-1,-1,-1,-1,-1,-1,-1,-1,-1,-1,-1,-1,-1,-1,-1,-1,-1$,

$0,0,0,0,0,0,0,0,0,0,0,0,0,0,0,0,0,0,0,0,0,0,0,0]$,

$[5$,

$-1,-1,-1,-1,-1,-1,-1,-1,-1,-1$,

$1,1,1,1,1,1,1,1,1,1,1,1,1,1,1$,

$-1,-1,-1,-1,-1,-1,-1,-1,-1,-1,-1,-1,-1,-1,-1,-1,-1,-1,-1,-1$,

$-1,-1,-1,-1,-1,-1,-1,-1,-1,-1,-1,-1,-1,-1,-1,-1,-1,-1,-1,-1$

$1,1,1,1,1,1,1,1,1,1,1,1,1,1,1,1,1,1,1,1,1,1,1,1,1,1,1,1,1,1$,

$0,0,0,0,0,0,0,0,0,0,0,0,0,0,0,0,0,0,0,0,0,0,0,0]$,

$[6$,

$0,0,0,0,0,0,0,0,0,0$,

$-2,-2,-2,-2,-2,-2,-2,-2,-2,-2,-2,-2,-2,-2,-2$, 


\section{Page 87 of 107}

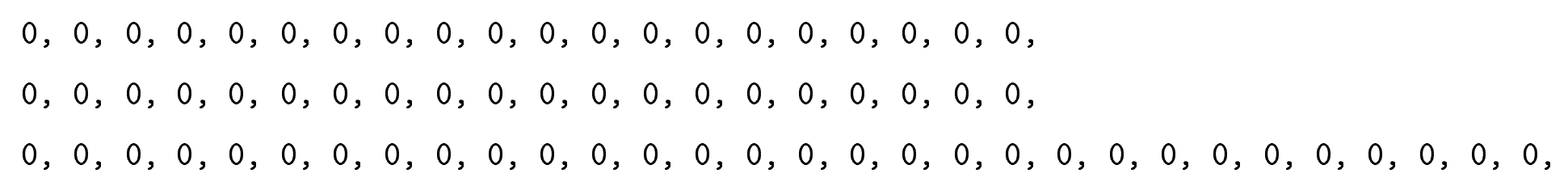




$$
\mathrm{v}[\mathrm{j}]:=\operatorname{key}[\mathrm{j}, \mathrm{k}]:
$$

od :

$$
\mathrm{u}:=\operatorname{linalg}[\mathrm{multiply}] \text { (pmat [nop] , v) : }
$$

for kk from 1 to kks do

SSS : $=0$ :

for $j$ from 1 to $m$ do sss : $=\operatorname{sss}+(\operatorname{key}[j, k k i[k k]]-u[j]) * * 2$ :

od:

if $(\mathrm{sss}=0)$ then TD [nop] [kki [kk], k] : =1 :

kks : $=k k s-1$ :

for kkk from kk to kks do

kki [kkk] : =kki [kkk+1] :

od :

break:

end if

od:

od :

od:

TD :

end proc: 


\section{Page 89 of 107}

\section{Molecular Physics}

redA1:= proc(ng, nirr, chartab, ndim, rep, runtime)

local nop, mu, k, l, km, chi, ss , redfac , T, NC, VC, GS , DT, dirrp, label, TT, ID , nD, repn, gammu:

description "calculation of symmetry reduction by projection":

chi : =array $(1 \ldots \mathrm{ng})$ :

redfac: $=\operatorname{array}(1$, nirr $)$ :

label : =array $(1 \ldots$ ndim) :

$\operatorname{dirrp}:=\operatorname{array}(1 \ldots \operatorname{nirr},[\operatorname{seq}(\operatorname{chartab}[\mathrm{k}, 1], \mathrm{k}=1 \ldots \operatorname{nirr})])$ : \#dimension of irrep

for nop from 1 to $\mathrm{ng}$ do \# get character of representation rep

chi $[$ nop $]:=0$ :

for $k$ from 1 to ndim do

chi [nop] :=chi [nop] +rep [nop] [k,k] :

od:

chi [nop] :=simplify (expand (chi[nop])) :

od :

for mu from 1 to nirr do \# calculation of reduction factors

Ss : $=0$ :

for nop from 1 to $\mathrm{ng}$ do

ss : $=$ sss + chi $[$ nop $] * \operatorname{chartab}[\mathrm{mu}, \mathrm{nop}]$ :

od:

sss : =simplify (expand (sss)) :

redfac $[\mathrm{mu}]:=\mathrm{sss} / \mathrm{ng}$ : 
if not type(redfac [mu], integer) then

print ("Reduction factor for irrep ",mu," is not integer."):

print ("Check representation. Program stopped."):

\#

stop

end if :

od:

for mu from 1 to 1 do \# calculate projection on A1 only

$\mathrm{T}:=$ Matrix (ndim, ndim, shape=zero):

for nop from 1 to ng do

repn:=matrix (ndim, ndim) :

repn :=eval (rep [nop]) :

$\mathrm{T}:=\operatorname{evalm}(\mathrm{T}+\operatorname{chartab}[\mathrm{mu}, \mathrm{nop}] *$ repn $)$ :

od :

gammu : $=\operatorname{redfac}[\mathrm{mu}] * \operatorname{dirrp}[\mathrm{mu}]$ :

GS:=orthonorm(ndim, ndim, $\mathrm{T}$, gammu, runtime): \# Procedure orthonorm returns 2 items:

NC $[\mathrm{mu}]:=\mathrm{GS}[1]: \quad$ \# number of non-zero vectors

VC [mu] : =evalm (GS [2] )

\# list of NC column vectors

\# of length ndim

$\# \operatorname{print}(\mathrm{NC}[\mathrm{mu}])$;

\#print (VC $[\mathrm{mu}]$ );

if $(\mathrm{NC}[\mathrm{mu}] \ll \operatorname{redfac}[\mathrm{mu}] * \operatorname{dirrp}[\mathrm{mu}])$ then 


\section{Page 91 of 107}

\section{Molecular Physics}

print("Mismatch in projection onto irrep space $\mathrm{mu}="$, $\mathrm{mu}$ ):

$\operatorname{print}(\mathrm{NC}[\mathrm{mu}], \operatorname{redfac}[\mathrm{mu}])$ :

error "Program stopped."

end if:

od:

$\mathrm{T}:=\operatorname{matrix}($ ndim, $\operatorname{redfac}[1])$ :

$\mathrm{k}:=0$ :

for mu from 1 to 1 do

for $\mathrm{km}$ from 1 to $\mathrm{NC}[\mathrm{mu}]$ do

$\mathrm{k}:=\mathrm{k}+1$ :

for 1 from 1 to ndim do

$\mathrm{T}[\mathrm{l}, \mathrm{k}]:=\mathrm{VC}[\mathrm{mu}][\mathrm{km}][\mathrm{l}]$ :

od:

od :

od :

redfac: $=\operatorname{eval}($ redfac, 4$)$ :

$\mathrm{T}:=\operatorname{eval}(\mathrm{T}, 4)$ :

[redfac, T] :

end proc:

redfac : $=$ proc (ng, nirr, chartab, labtab, ndim, rep)

local nop, mu, k, l, km, chi , ss , redf , T, NC, VC, GS , DT , dirrp, label, TT, ID ,nD, repn:

URL: http://mc.manuscriptcentral.com/tandf/tmph 
description "calculation of symmetry reduction by projection":

chi :=array $(1 \ldots \mathrm{ng})$ :

redf : =array (1..nirr) :

label :=array (1..ndim) :

$\operatorname{dirrp}:=\operatorname{array}(1 \ldots \operatorname{nirr},[\operatorname{seq}(\operatorname{chartab}[\mathrm{k}, 1], \mathrm{k}=1 \ldots \mathrm{nirr})])$ : \#dimension of irrep

for nop from 1 to $\mathrm{ng}$ do \# get character of representation rep

chi $[\mathrm{nop}]:=0$ :

for $\mathrm{k}$ from 1 to ndim do

chi [nop] :=chi [nop] + rep $[n o p][k, k]$ :

od :

$\operatorname{chi}[$ nop] :=simplify (expand ( $\operatorname{chi}[$ nop] $))$ :

od:

for mu from 1 to nirr do \# calculation of reduction factors SsS : $=0$ :

for nop from 1 to $\mathrm{ng}$ do

sss : $=$ sss $+\operatorname{chi}[$ nop] $* \operatorname{chartab}[\mathrm{mu}, \mathrm{nop}]$ :

od :

sss : =simplify (expand (sss)) :

redf $[\mathrm{mu}]:=\mathrm{sss} / \mathrm{ng}$ :

if not type(redf [mu], integer) then

print("Reduction factor for irrep ",mu," is not integer."); 
end if:

od:

redf :

end proc:

reduce: $=\operatorname{proc}(m u$, gammu , ng , Gi, chartab , ndim , G, runtime)

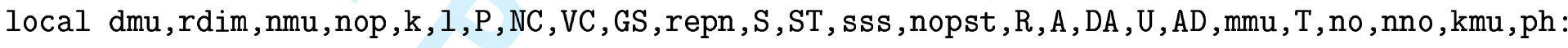

description "calculation of symmetry reduction in subspace mu by projection; hammermesh idea": $\mathrm{dmu}:=\operatorname{eval}(\operatorname{chartab}[\mathrm{mu}, 1])$ : \#dimension of irrep mu no: $=6000$ :

○ $\operatorname{rdim}:=\mathrm{dmu} *$ gammu :

$\mathrm{nmu}:=1$ :

nopst: $=1$ :

$P:=$ Matrix (ndim, ndim, shape=zero):

for nop from 1 to $\mathrm{ng}$ do

$\mathrm{P}:=\operatorname{evalm}(\mathrm{P}+\mathrm{dmu} / \mathrm{ng} * \operatorname{eval}(\mathrm{Gi}[\mathrm{mu}][\mathrm{nop}][\mathrm{nmu}, \mathrm{nmu}]) * \mathrm{G}[\mathrm{nop}])$ :

od:

$\# \operatorname{print}(\mathrm{P})$;

GS:=orthonorm(ndim, ndim, P, gammu, runtime): \# Procedure orthonorm returns 2 items:

$$
\text { NC:=GS[1]: \# number of non-zero vectors }
$$

VC:=evalm(GS[2]): \# list of NC column vectors 


\section{\# of length ndim}

unassign ('GS') :

\#print (NC) ;

\#print (VC);

if $(\mathrm{NC}<>$ gammu) then

print("Mismatch in projection onto irrep space $\mathrm{mu}="$ ", mu):

print (NC, gammu):

error "Program stopped."

end if :

$\mathrm{S}[\mathrm{nmu}]:=\operatorname{matrix}($ ndim, gammu):

If for $\mathrm{k}$ from 1 to $\mathrm{NC}$ do

for 1 from 1 to ndim do

$\mathrm{S}[\mathrm{nmu}][1, \mathrm{k}]:=\mathrm{VC}[\mathrm{k}][1]$

od :

od :

nno: $=$ no+1 :

prtime(outputfile,nno, "REDUCE: get S1",runtime):

\# $\quad \operatorname{print}(\mathrm{S}[\mathrm{nmu}])$;

for $\mathrm{mmu}$ from 2 to dmu do

P:=Matrix (ndim, ndim, shape=zero):

for nop from 1 to ng do 


\section{Page 95 of 107}

\section{Molecular Physics}

$\mathrm{P}:=\operatorname{evalm}(\mathrm{P}+\mathrm{dmu} / \mathrm{ng} * \operatorname{eval}(\mathrm{Gi}[\mathrm{mu}][\mathrm{nop}][\mathrm{mmu}, \mathrm{nmu}]) * \mathrm{G}[\mathrm{nop}]):$

od:

$\mathrm{S}[\mathrm{mmu}]:=\operatorname{evalm}(\mathrm{P} \& * \mathrm{~S}[\mathrm{nmu}])$ :

\# for $\mathrm{k}$ from 1 to gammu do

\#normalize S

\# VC:=array $(1 \ldots \operatorname{ndim},[\operatorname{seq}(\mathrm{S}[\mathrm{mmu}][1, \mathrm{k}], \mathrm{l}=1 \ldots \mathrm{ndim})])$ :

\# NC $[\mathrm{k}]:=\operatorname{vecnorm}(\mathrm{VC})$ :

$\#$ if $(\mathrm{NC}[\mathrm{k}]=0)$ then

\# error "trivial projection stop"

\# end if:

\# od:

for $k$ from 1 to gammu do

for 1 from 1 to ndim do

\# $\quad \mathrm{S}[\mathrm{mmu}][1, \mathrm{k}]:=\operatorname{combine}(\mathrm{S}[\mathrm{mmu}][1, \mathrm{k}] / \mathrm{NC}[\mathrm{k}]$, radical, symbolic $)$ : $\mathrm{S}[\mathrm{mmu}][1, \mathrm{k}]:=$ combine $(\mathrm{S}[\mathrm{mmu}][1, \mathrm{k}]$, radical, symbolic) :

od

od :

\# $\quad \operatorname{print}(\mathrm{S}[\mathrm{nmu}])$;

nno: $=$ no+nmu

prtime(outputfile,nno, "REDUCE: get $\mathrm{Si}, \mathrm{i}>1$ ", runtime):

od :

$\mathrm{T}:=\operatorname{matrix}($ ndim, $r d i m)$ : 
$\mathrm{k}:=0$ :

for $\mathrm{mmu}$ from 1 to gammu do

A : =matrix (ndim, dmu) :

for $\mathrm{nmu}$ from 1 to dmu do

for 1 from 1 to ndim do

$\mathrm{A}[1, \mathrm{nmu}]:=\mathrm{S}[\mathrm{nmu}][1, \mathrm{mmu}]$ :

od:

od:

ST : =linalg [transpose] (A) :

\# $\operatorname{print}(G[$ nopst]) :

GS : = simtra (A, ST, G [nopst $])$ :

GS : =eval (GS) :

\# $\quad \operatorname{print}(\mathrm{GS}, \mathrm{Gi}[\mathrm{mu}]$ [nopst]):

for $\mathrm{nmu}$ from 1 to $\mathrm{dmu}$ do

$\mathrm{ph}[\mathrm{nmu}]:=1$ :

od :

for $\mathrm{nmu}$ from 1 to $\mathrm{dmu}$ do

$\mathrm{k}:=\mathrm{k}+1$ :

for $\mathrm{kmu}$ from 1 to $\mathrm{dmu}$ do \#phase determination

if $(\mathrm{kmu} \longleftrightarrow>\mathrm{nmu})$ then \#just for testing

ss : =eval (Gi [mu] [nopst] [nmu, kmu] ,4) : 


\section{Page 97 of 107}

\section{Molecular Physics}

if $(\mathrm{sss}<>0)$ then

$\operatorname{ph}[\mathrm{nmu}]:=\mathrm{ph}[\mathrm{nmu}] * \mathrm{ph}[\mathrm{kmu}] * \operatorname{eval}(\mathrm{GS}[\mathrm{nmu}, \mathrm{kmu}], 4) / \mathrm{sss}:$

break:

end if:

end if:

od:

\# $\operatorname{print}(\mathrm{mmu}, \mathrm{nmu}, \mathrm{kmu}, \mathrm{sss}, \mathrm{ph}[\mathrm{nmu}])$;

if (evalf $(\mathrm{ph}[\mathrm{nmu}] * * 2)<>1)$ then

error "Phase error in reduce; program stopped"

end if :

of for 1 from 1 to ndim do

$\mathrm{T}[1, \mathrm{k}]:=\mathrm{A}[1, \mathrm{nmu}] * \mathrm{ph}[\mathrm{nmu}]:$

\# $\quad \mathrm{T}[1, \mathrm{k}]:=\mathrm{S}[\mathrm{nmu}][1, \mathrm{mmu}]$ :

od:

od :

od:

if $(\mathrm{k}<>$ rdim) then

error "Mismatch in reduce: nb of columns $<>$ rdim; program stopped."

end if:

$\mathrm{T}:=\operatorname{map}$ (combine, $\mathrm{T}$, radical, symbolic) :

end proc: 


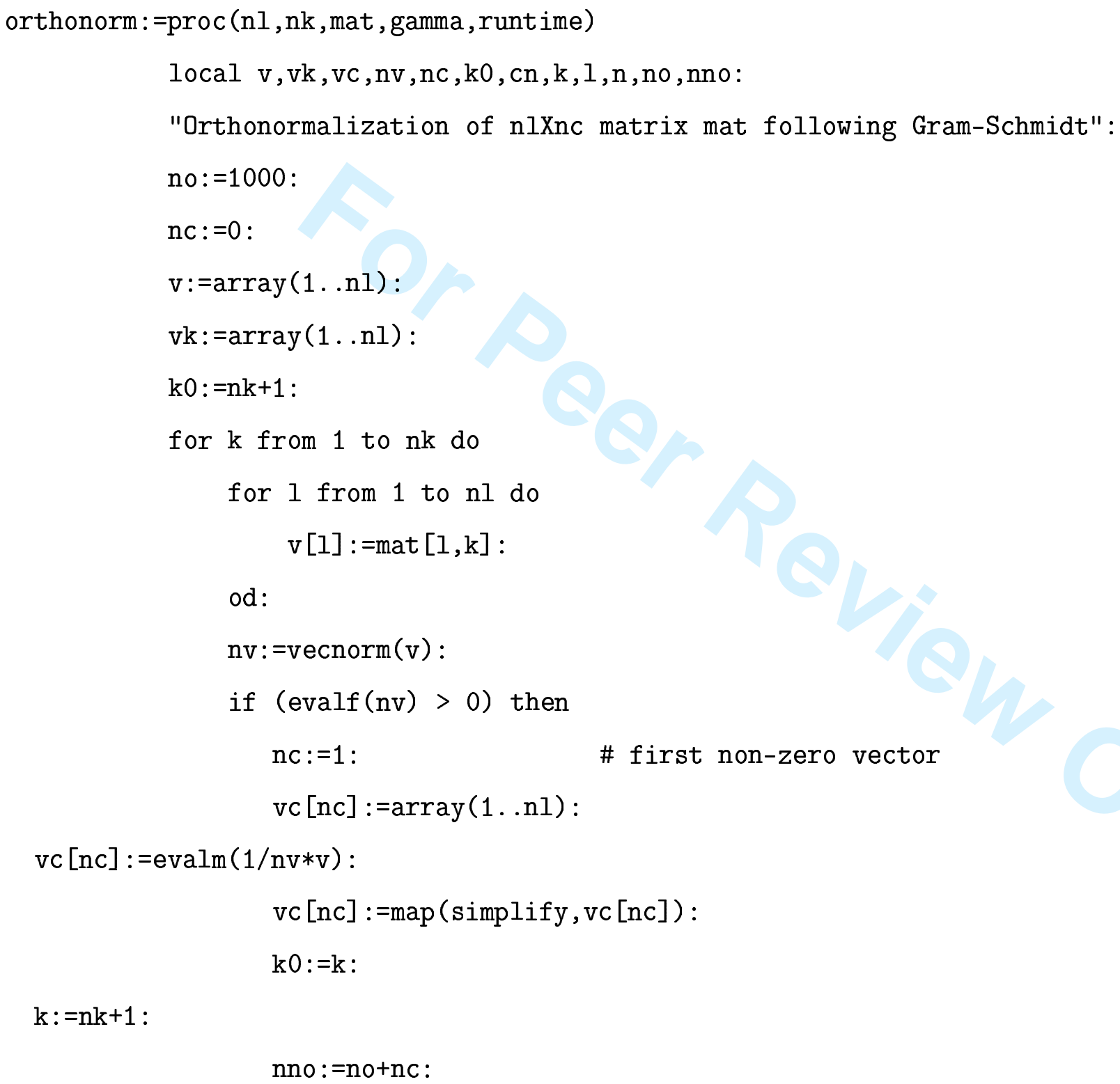




\section{Page 99 of 107}

\section{Molecular Physics}

prtime (outputfile, nno, "ORTHONORM ", runtime):

end if:

od:

for $k$ from $(k 0+1)$ to $n k$ do

if (nc=gamma) then

break:

end if:

for 1 from 1 to $\mathrm{nl}$ do

$\operatorname{vk}[1]:=\operatorname{mat}[\mathrm{l}, \mathrm{k}]$ :

$\mathrm{v}[\mathrm{l}]:=\mathrm{vk}[\mathrm{l}]$ :

9

for $\mathrm{n}$ from 1 to $\mathrm{nc}$ do

$\mathrm{cn}:=\operatorname{evalm}(\mathrm{vk} \& * \mathrm{vc}[\mathrm{n}]):$

$\mathrm{v}:=\operatorname{evalm}(\mathrm{v}-\mathrm{cn} * \mathrm{vc}[\mathrm{n}])$ :

$\mathrm{v}:=\operatorname{map}(\mathrm{simplify}, \mathrm{v})$ :

od:

$\mathrm{nv}:=\mathrm{vecnorm}(\mathrm{v}):$

if (evalf (nv) $<>0)$ then

$\mathrm{nc}:=\mathrm{nc}+1$

$\mathrm{vc}[\mathrm{nc}]:=\operatorname{array}(1 \ldots \mathrm{nl})$ :

$\mathrm{vc}[\mathrm{nc}]:=\operatorname{evalm}(1 / \mathrm{nv} * \mathrm{v})$ :

URL: http://mc.manuscriptcentral.com/tandf/tmph 


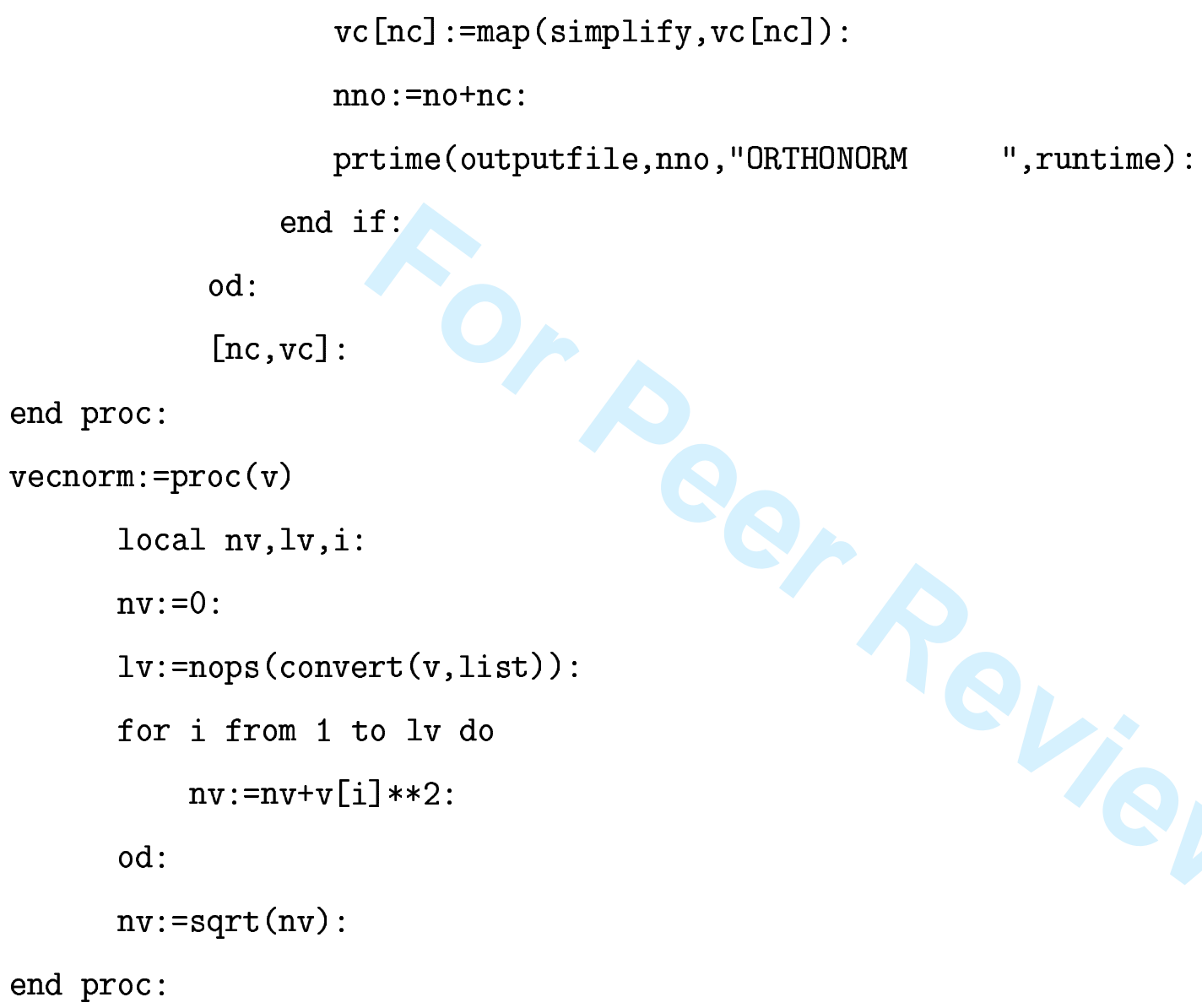




\section{D sample input}

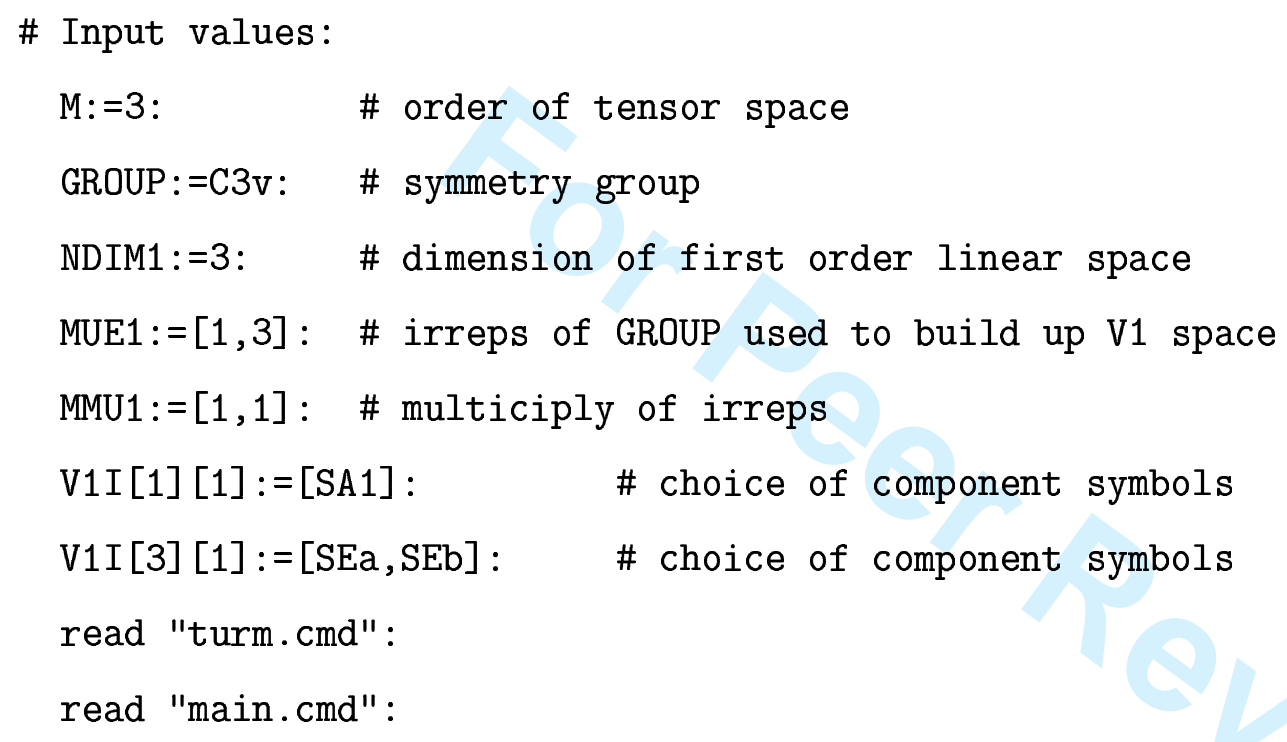




\section{E sample output}

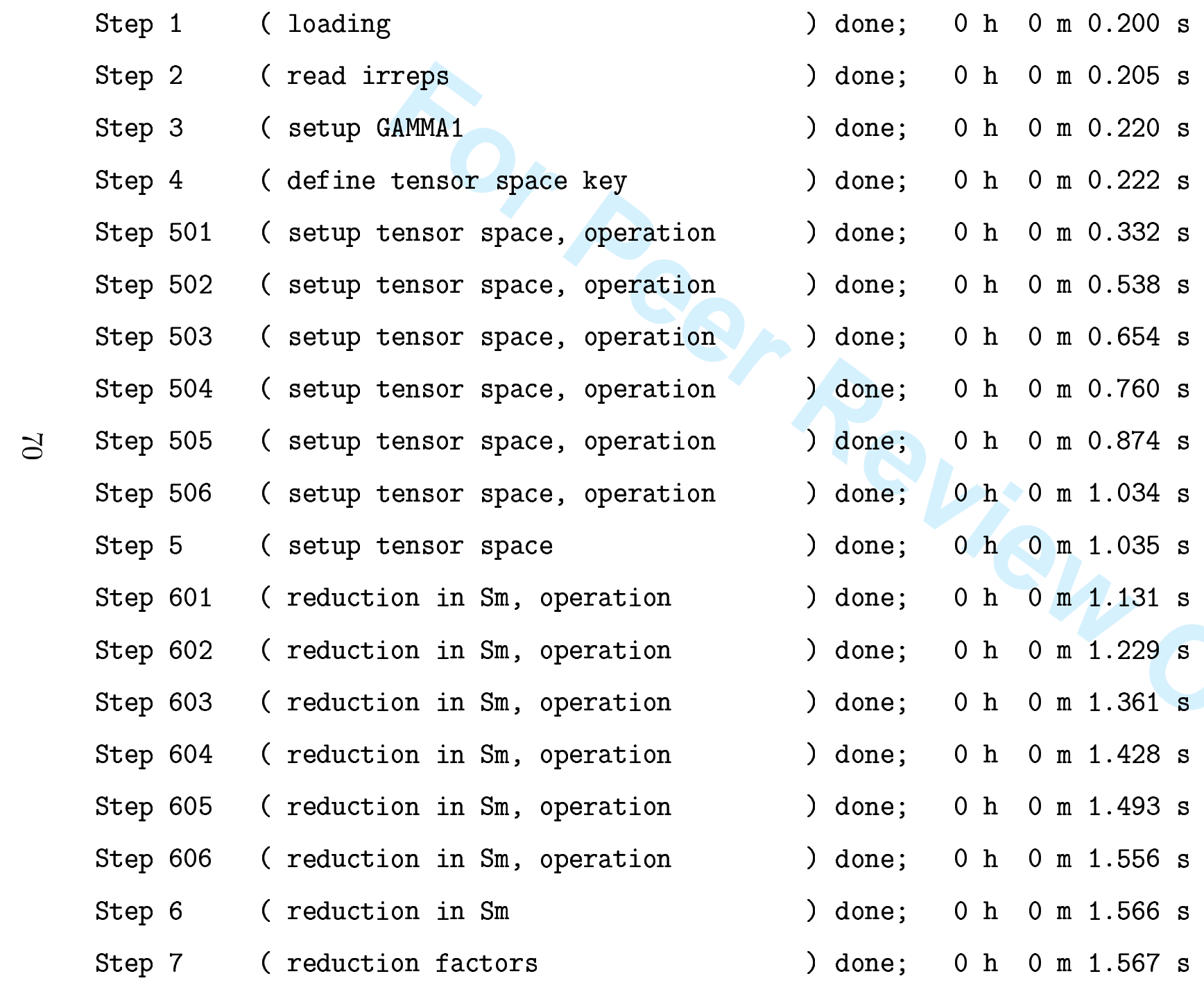




\begin{tabular}{|c|c|c|c|}
\hline Step 1001 & \multicolumn{3}{|l|}{ ( ORTHONORM } \\
\hline Step 1002 & \multicolumn{3}{|l|}{ ( ORTHONORM } \\
\hline Step 1003 & \multicolumn{3}{|l|}{ ( ORTHONORM } \\
\hline Step 6001 & \multicolumn{3}{|l|}{ ( REDUCE: get S1 } \\
\hline Step 1001 & \multicolumn{3}{|l|}{ ( ORTHONORM } \\
\hline Step 6001 & \multicolumn{3}{|l|}{ ( REDUCE: get $\mathrm{S} 1$} \\
\hline Step 1001 & \multicolumn{3}{|l|}{ ( ORTHONORM } \\
\hline Step 1002 & \multicolumn{3}{|l|}{ ( ORTHONORM } \\
\hline Step 1003 & \multicolumn{3}{|l|}{ ( ORTHONORM } \\
\hline Step 6001 & \multicolumn{3}{|l|}{ ( REDUCE: get $\mathrm{S} 1$} \\
\hline Step 6001 & \multicolumn{3}{|c|}{ ( REDUCE: get $\mathrm{Si}, i>1$} \\
\hline Step 8 & \multicolumn{3}{|l|}{ ( reduce GAMMAmr } \\
\hline Step 9 & \multicolumn{3}{|c|}{ ( tensor transformations } \\
\hline type $A 1$ & , operation 1 & , difference & 0.0 \\
\hline type $A 1$ & , operation 2 & , difference & 0.0 \\
\hline type $A 1$ & , operation 3 & , difference & 0.0 \\
\hline type A1 & , operation 4 & , difference & 0.0 \\
\hline type A1 & , operation 5 & , difference & 0.0 \\
\hline type A1 & , operation 6 & , difference & 0.0 \\
\hline type A2 & , operation 1 & , difference & 0.0 \\
\hline type $A 2$ & , operation 2 & , difference & \\
\hline
\end{tabular}

) done; $0 \mathrm{~h} 0 \mathrm{~m} 1.688 \mathrm{~s}$

) done; $0 \mathrm{~h} 0 \mathrm{~m} 1.713 \mathrm{~s}$

) done; $0 \mathrm{~h} 0 \mathrm{~m} 1.756 \mathrm{~s}$

) done; $0 \mathrm{~h} 0 \mathrm{~m} 1.757 \mathrm{~s}$

) done; $0 \mathrm{~h} 0 \mathrm{~m} 1.875 \mathrm{~s}$

) done; $0 \mathrm{~h} 0 \mathrm{~m} 1.875 \mathrm{~s}$

) done; $0 \mathrm{~h} 0 \mathrm{~m} 2.008 \mathrm{~s}$

) done; $0 \mathrm{~h} 0 \mathrm{~m} 2.027 \mathrm{~s}$

) done; $0 \mathrm{~h} 0 \mathrm{~m} 2.068 \mathrm{~s}$

) done; $0 \mathrm{~h} 0 \mathrm{~m} 2.069 \mathrm{~s}$

) done; $0 \mathrm{~h} 0 \mathrm{~m} \mathrm{2.152 \textrm {s }}$

) done; $0 \mathrm{~h} 0 \mathrm{~m} \mathrm{2.193 \textrm {s }}$

) done; $0 \mathrm{~h} 0 \mathrm{~m} 2.703 \mathrm{~s}$

URL: http://mc.manuscriptcentral.com/tandf/tmph 
type A2, operation 3 , difference 0.0

type $\mathrm{A} 2$, operation 4 , difference 0.0

type $A 2$, operation 5 , difference 0.0

type A2, operation 6 , difference 0.0

type $\mathrm{E}$, operation 1 , difference 0.0

type $\mathrm{E}$, operation 2 , difference 0.0

type $\mathrm{E}$, operation 3 , difference 0.0

type $\mathrm{E}$, operation 4 , difference 0.0

type $\mathrm{E}$, operation 5 , difference 0.0

type $\mathrm{E}$, operation 6 , difference 0.0

N

Final results:

1) Original tensor space dimension: 27

Reduced tensor space dimension: 10

2) Reduction factors:

LABEL [mu] GAMMA [mu]

$\begin{array}{ll}\text { A1 } & 3 \\ \text { A2 } & 1\end{array}$


3) Symmetrized expressions:

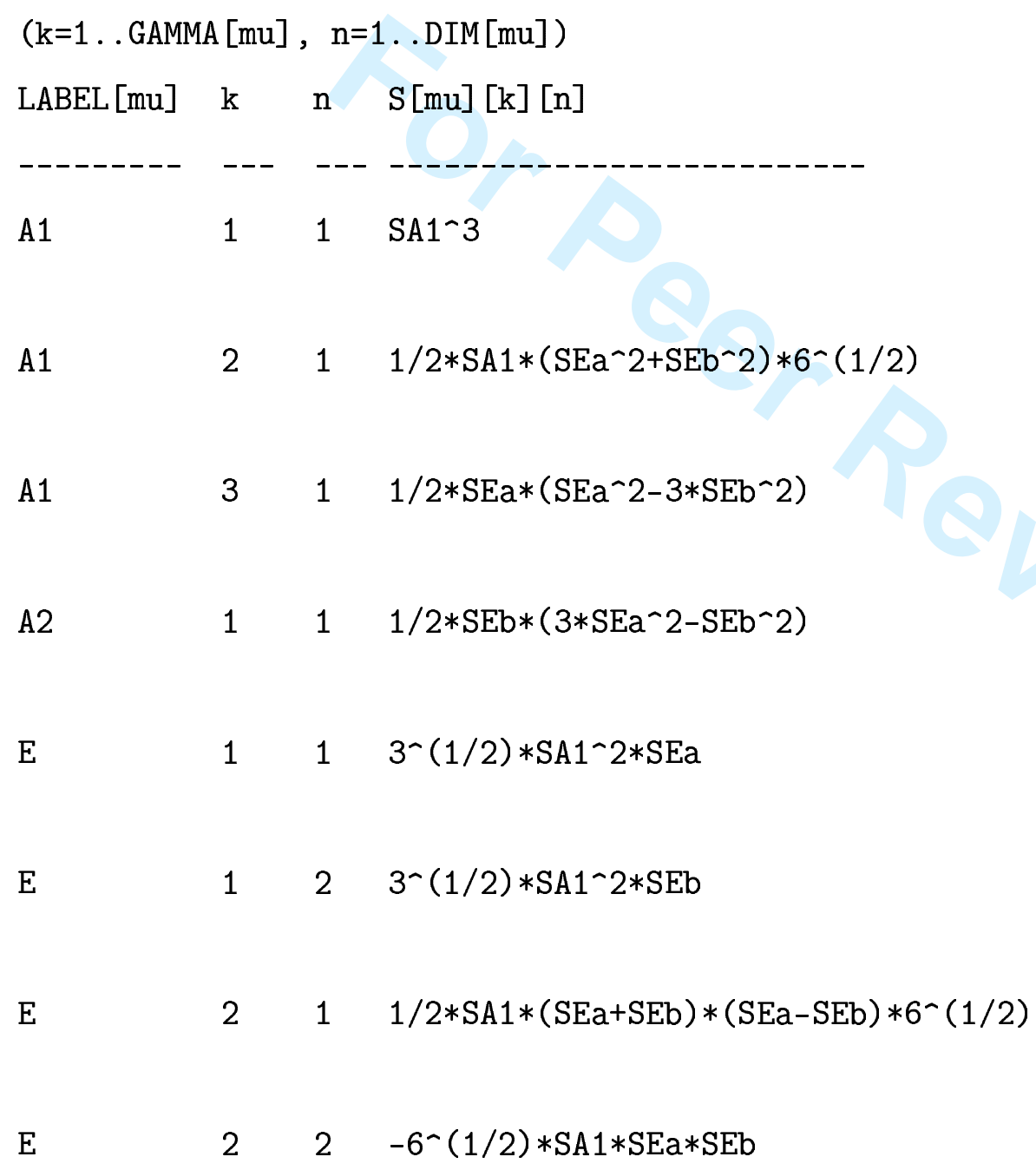


$311 / 2 * 3 \sim(1 / 2) *\left(\mathrm{SEa} \_2+\mathrm{SEb} \_2\right) * \mathrm{SEa}$

E

$321 / 2 * 3-(1 / 2) *\left(\mathrm{SEa}^{\wedge} 2+\mathrm{SEb} \_2\right) * \mathrm{SEb}$

Step $11(* * * * * * * * * * * * * *))$ done; $0 \mathrm{~h} \quad 0 \mathrm{~m} 2.928 \mathrm{~s}$ 


\section{F unix script}

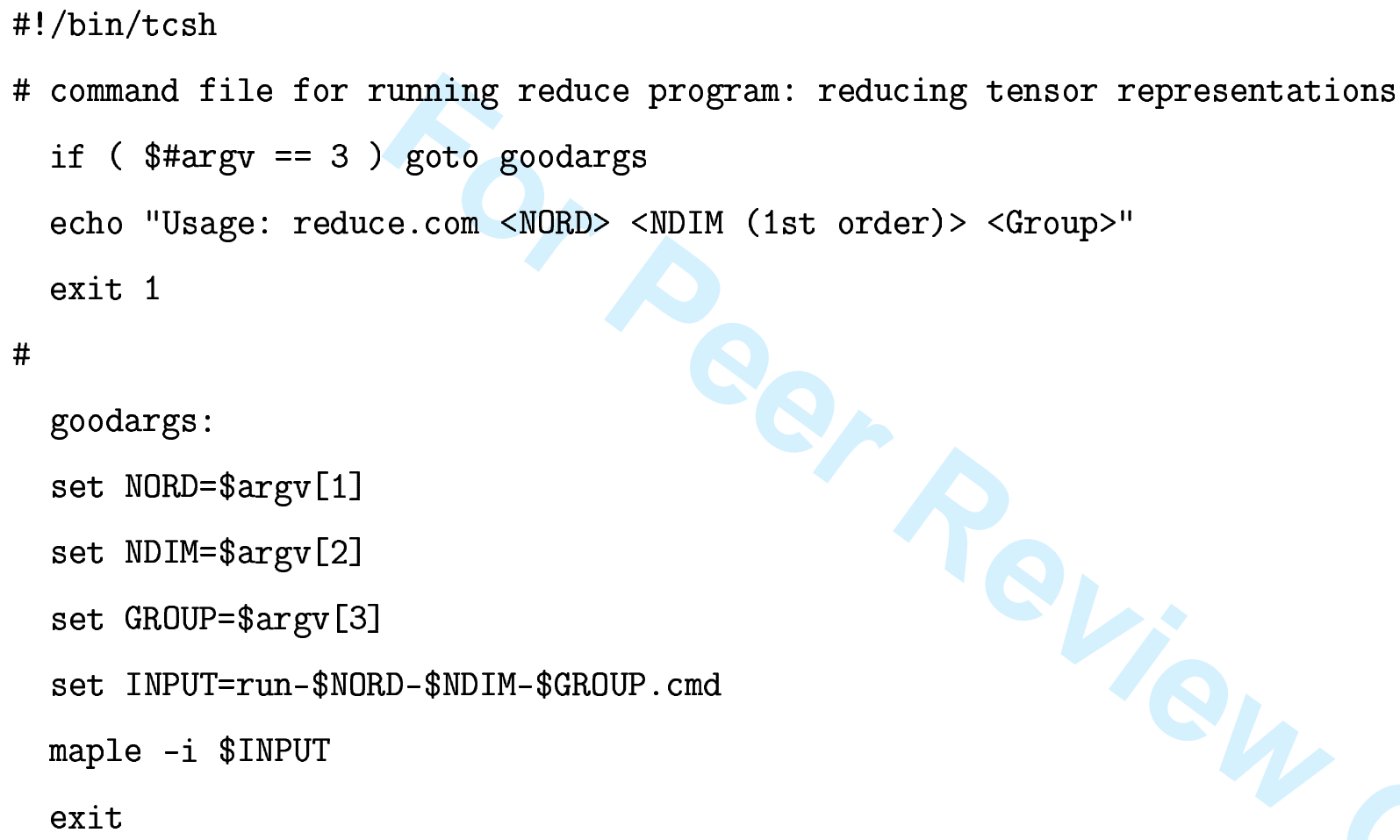

